hep-th/0110121

October, 2001

\title{
Exceptional String: Instanton Expansions and Seiberg-Witten Curve
}

\author{
Kenji Mohri* \\ Institute of Physics, University of Tsukuba \\ Ibaraki 305-8571, Japan
}

\begin{abstract}
We investigate instanton expansions of partition functions of several toric Estring models using local mirror symmetry and elliptic modular forms. We also develop a method to determine the Seiberg-Witten curve of E-string with the help of elliptic functions.
\end{abstract}

${ }^{*}$ E-mail address: mohri@het.ph.tsukuba.ac.jp 


\section{Contents}

1 Introduction $\quad 2$

2 E-String $\quad 3$

2.1 Homology lattice and affine root lattice . . . . . . . . . . . . . 3

2.2 Partition functions . . . . . . . . . . . . . . . . . . 6

3 Four Torus Models $\quad 10$

3.1 Periods of elliptic curves . . . . . . . . . . . . . . . . . 10

3.2 Modular identities . . . . . . . . . . . . . . . . . 13

4 Model Building of E-Strings $\quad 14$

4.1 Principal series . . . . . . . . . . . . . . . . . . . 14

$4.2 \boldsymbol{E}_{0}$ and $\boldsymbol{E}_{\tilde{1}}$ models $\ldots \ldots \ldots \ldots \ldots \ldots$

4.3 Kähler classes . . . . . . . . . . . . . . . . . . . . 16

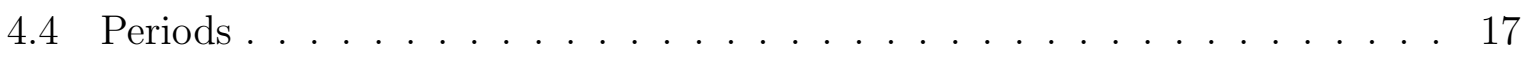

4.5 Relation to Seiberg-Witten periods . . . . . . . . . . . . . . . 21

5 Genus Zero Partition Functions 23

5.1 Recursion relations . . . . . . . . . . . . . . . . . 23

5.2 Instanton expansion $\ldots \ldots \ldots \ldots$

5.3 Partition functions and modular forms . . . . . . . . . . . . . . 28

5.4 Modular anomaly equation . . . . . . . . . . . . . . . . 30

5.5 Rational instanton numbers . . . . . . . . . . . . . . . . 30

6 Genus One Partition Functions $\quad 34$

6.1 Genus one potentials . . . . . . . . . . . . . . . . 34

6.2 Modular anomaly equation . . . . . . . . . . . . . . . . 38

6.3 Elliptic instanton numbers . . . . . . . . . . . . . . . . . . . . 38

7 Higher Genus Partition Functions $\quad 42$

7.1 Partition functions as modular forms . . . . . . . . . . . . . . . . . . 42 
7.2 Gopakumar-Vafa invariants . . . . . . . . . . . . . . . . . 46

7.3 Partition functions as Jacobi forms . . . . . . . . . . . . . . 51

8 Seiberg-Witten Curve $\quad 54$

8.1 Periods of rational elliptic surfaces . . . . . . . . . . . . . . 54

8.2 Wilson lines . . . . . . . . . . . . . . . . . . . . . 58

9 Inverse Problem $\quad 59$

9.1 General strategy . . . . . . . . . . . . . . . . . . . . . . . 59

9.2 Several models with a few Wilson lines . . . . . . . . . . . . . . . . 62

$\begin{array}{lr}\text { Appendix A } & 66\end{array}$

\section{Introduction}

Exceptional string (E-string in short) has originally been discovered as the effective six dimensional (6D) theory associated with a small $\boldsymbol{E}_{8}$ instanton in heterotic string [11]. It has become clear since then that physical content of the toroidal compactification of E-string down to 4D is extremely rich [10, 12, 23, 24, 29, 27, 18].

4D E-string theory can be realized as type IIA string "compactified" on the canonical line bundle of a rational elliptic surface $B_{9}$. However we must rely on mirror symmetry for any quantitative analysis. In fact, we have two versions of mirror symmetry. One is local mirror symmetry [6] applied to $B_{9}$, which must be realized torically [16]. At the expense of the restriction on Kähler moduli, this method enables us to investigate systematically the BPS spectrum even at higher genera [18]. The other describes E-string by the Seiberg-Witten curve $[10,12]$ based on the fact that $B_{9}$ is self-mirror; the E-string is mapped to type IIB string on a non-compact Calabi-Yau manifold containing a rational elliptic surface $S_{9}$ [27], the complex moduli of which replaces the Kähler moduli of $B_{9}$. The purpose of this paper is then to explore further these two descriptions of E-string.

This paper is organized as follows. In section 2, we collect general results on the Kähler moduli parameters and the partition functions of E-string. The remaining sections are divided into two parts. The first part consists of sections 3-7, where we investigate the 
six toric E-string models by means of local mirror symmetry; in section 3, we study the four torus models associated with the E-string models, where the relation between the periods and elliptic modular forms is the central problem, in section 4 we analyze the Picard-Fuchs system of the E-string models; we then investigate the partition functions of the E-string models of genus zero, one in sections 5, 6 respectively; finally in section 7, partition functions of higher genera are considered in connection with elliptic modular forms, Gopakumar-Vafa invariants and $\boldsymbol{E}_{8}$ Jacobi forms.

The second part deals with the Seiberg-Witten curve of E-string; after a review of the period map of rational elliptic surface in section 8, we obtain a procedure to determine the Seiberg-Witten curve for given Wilson lines using elliptic functions in section 9.

\section{$2 \quad$ E-String}

\subsection{Homology lattice and affine root lattice}

Let us first consider type IIA string compactified on a Calabi-Yau threefold which contains a rational elliptic surface $B_{9}$ with a section [30] as a divisor, where we use the symbol $B_{N}$ for $\boldsymbol{E}_{N}$ del Pezzo surface; a rational elliptic surface is alternatively called an almost $\boldsymbol{E}_{9}$ del Pezzo surface.

The resulting $4 \mathrm{D}$ theory is a $N=2$ supergravity. The large radius limit of the normal direction to $B_{9}$ then kills almost all the degrees of freedom of the original Calabi-Yau threefold; effectively we are left with $K_{B_{9}}$, the canonical line bundle of $B_{9}$, as a compactification manifold, and the 4D theory reduces to a E-string theory which does not contain gravity. We are interested in the physical quantities of the E-string that depend only on the complexified Kähler class $J \in H_{2}\left(B_{9} ; \mathbf{C}\right)$ of $B_{9}$ inherited from the Calabi-Yau threefold. It should be noted that we can take the complex structure of $B_{9}$ to be generic, that is, we can assume that the elliptic fibration $\pi: B_{9} \rightarrow \mathbf{P}^{1}$ has the twelve singular fibers of the $\mathrm{I}_{1}$ type.

We recall here some properties of the second homology classes $H_{2}\left(B_{9}\right)$. The lattice 
structure in $H_{2}\left(B_{9}\right)$ induced by intersection parings is given by

$$
\begin{aligned}
H_{2}\left(B_{9}\right) & =\mathbf{Z} l \oplus \mathbf{Z} \mathcal{E}_{1} \oplus \cdots \oplus \mathbf{Z} \mathcal{E}_{9}, \\
l \cdot l & =1, \quad l \cdot \mathcal{E}_{i}=0, \quad \mathcal{E}_{i} \cdot \mathcal{E}_{j}=-\delta_{i j} .
\end{aligned}
$$

$B_{9}$ is realized as a blow-up of $\mathbf{P}^{2}$ at the nine points which are the base points of a cubic pencil. The class $l$ is given by the total transform of a line in $\mathbf{P}^{2}$, while $\mathcal{E}_{i}$ the exceptional divisor associated with the $i$ th base point. The first Chern class $c_{1}\left(B_{9}\right)$ is represented by the fiber class $[\delta]$ of the elliptic fibration $\pi: B_{9} \rightarrow \mathbf{P}^{1}$, which can be written in terms of the generators $(2.2)$ as $[\delta]=3 l-\mathcal{E}_{1}-\cdots-\mathcal{E}_{9}$. It is readily verified that $[\delta] \cdot l=3$, $[\delta] \cdot \mathcal{E}_{i}=1$, and $[\delta] \cdot[\delta]=0$.

The sublattice $[\delta]^{\perp}$ of $H_{2}\left(B_{9}\right)$, which is the orthogonal complement of $[\delta]$ in $H_{2}\left(B_{9}\right)$, is naturally identified with the root lattice of the affine Lie algebra $\boldsymbol{E}_{8}^{(1)}: L\left(\boldsymbol{E}_{8}^{(1)}\right)=$ $\bigoplus_{i=0}^{8} \mathbf{Z} \alpha_{i}$, where $\left\{\alpha_{i}\right\}_{i=0}^{8}$ is the simple roots of $\boldsymbol{E}_{8}^{(1)}$. This can be seen as follows. First we find the generators of $[\delta]^{\perp}$ as a free $\mathbf{Z}$-module

$$
\begin{gathered}
{[\delta]^{\perp}=\bigoplus_{i=0}^{8} \mathbf{Z}\left[\alpha_{i}\right], \quad H_{2}\left(B_{9}\right) \cong[\delta]^{\perp} \oplus \mathbf{Z} \mathcal{E}_{9},} \\
{\left[\alpha_{0}\right]=\mathcal{E}_{8}-\mathcal{E}_{9}, \quad\left[\alpha_{i}\right]=\mathcal{E}_{i}-\mathcal{E}_{i+1}, \quad i=1, \ldots, 7, \quad\left[\alpha_{8}\right]=l-\mathcal{E}_{1}-\mathcal{E}_{2}-\mathcal{E}_{3} .}
\end{gathered}
$$

Then we see that the intersection pairings $\left(\left[\alpha_{i}\right] \cdot\left[\alpha_{j}\right]\right)$ coincides with the minus of the Cartan matrix of $\boldsymbol{E}_{8}^{(1)}$. Note that the element of $L\left(\boldsymbol{E}_{8}^{(1)}\right)$ corresponding to $[\delta] \in[\delta]^{\perp}$ is

$$
[\delta]=\left[\alpha_{0}\right]+2\left[\alpha_{1}\right]+4\left[\alpha_{2}\right]+6\left[\alpha_{3}\right]+5\left[\alpha_{4}\right]+4\left[\alpha_{5}\right]+3\left[\alpha_{6}\right]+2\left[\alpha_{7}\right]+3\left[\alpha_{8}\right],
$$

which is in accord with the standard notation of the affine Lie algebra; $\alpha_{0}=\delta-\theta$, with $\theta$ the highest root of $\boldsymbol{E}_{8}$.

Let $\left\{\omega_{i}\right\}_{i=1}^{8}$ be the fundamental weights of $\boldsymbol{E}_{8}$. Then $\left(\omega_{i} \mid \alpha_{j}\right)=\delta_{i, j}$ and $\theta=\omega_{7}$. We denote the corresponding elements of $[\delta]^{\perp}$ by $\left\{\left[\omega_{i}\right]\right\}_{i=1}^{8}$, which satisfy $\left[\omega_{i}\right] \cdot\left[\alpha_{j}\right]=-\delta_{i, j}$.

The dual of the Cartan subalgebra of $\boldsymbol{E}_{8}^{(1)}$, which we denote by $\mathfrak{h}^{*}$, contains the zeroth fundamental weight $\Lambda_{0}$ as a generator in addition to the simple $\operatorname{roots}\left\{\alpha_{i}\right\}$, that is,

$$
\mathfrak{h}^{*}=\mathbf{C} \Lambda_{0} \oplus \mathbf{C} \delta \oplus \mathbf{C} \alpha_{1} \oplus \cdots \oplus \mathbf{C} \alpha_{8}
$$


$\Lambda_{0}$ satisfies $\left(\Lambda_{0} \mid \alpha_{i}\right)=0, i \neq 0$ and $\left(\Lambda_{0} \mid \delta\right)=1$, which leads to the final identification $\mathcal{E}_{9}=-\left[\Lambda_{0}\right]-1 / 2[\delta]$. Thus we have another basis of the vector space

$$
H_{2}\left(B_{9} ; \mathbf{Q}\right)=\bigoplus_{i=1}^{8} \mathbf{Q}\left[\alpha_{i}\right] \oplus \mathbf{Q}[\delta] \oplus \mathbf{Q}\left[\Lambda_{0}\right]
$$

from which it follows that $H_{2}\left(B_{9} ; \mathbf{C}\right)$ can be identified with $\mathfrak{h}^{*}$, the CSA of $\boldsymbol{E}_{8}^{(1)}$.

To summarize, we find the isomorphism: $\mathfrak{h}^{*} \ni x \mapsto[x] \in H_{2}\left(B_{9} ; \mathbf{C}\right)$ of the $\mathbf{C}$-vector spaces such that $(x \mid y)=-[x] \cdot[y]$ for any $x, y \in \mathfrak{h}^{*}$.

The important consequence on the above observation is that the Weyl group $W\left(\boldsymbol{E}_{8}^{(1)}\right)$ of $\boldsymbol{E}_{8}^{(1)}$ acts on $H_{2}\left(B_{9} ; \mathbf{C}\right)$. To see the effect of the Weyl action on the Kähler moduli parameters of the E-string, which should be a physical symmetry, we put the Kähler class $J \in H_{2}\left(B_{9} ; \mathbf{C}\right) \cong \mathfrak{h}^{*}$ in the canonical form

$$
J=\left(\frac{1}{2} \tau+\sigma\right)[\delta]-\tau\left[\Lambda_{0}\right]-\sum_{i=1}^{8} \mu_{i}\left[\omega_{i}\right],
$$

where $\tau$ is the complex modulus of the torus $\mathbf{T}^{2}$, on which the $6 \mathrm{D}$ E-string theory is compactified to $4 \mathrm{D}, \tau+\sigma$ the Kähler modulus of $\mathbf{T}^{2}$, or equivalently, the E-string tension and the self-dual $B$-flux on $\mathbf{T}^{2}$, and $\left(\mu_{i}\right)$ the $\boldsymbol{E}_{8}$ Wilson lines, that is, the moduli of the flat $\boldsymbol{E}_{8}$ bundles on $\mathbf{T}^{2}[10,12]$.

There exists a semi-direct product structure: $W\left(\boldsymbol{E}_{8}^{(1)}\right)=W\left(\boldsymbol{E}_{8}\right) \times T$, where $W\left(\boldsymbol{E}_{8}\right)$ is the finite Weyl group and $T:=\left\{t_{\beta} \mid \beta \in L\left(\boldsymbol{E}_{8}\right)\right\}$, the translation by the root lattice $L\left(\boldsymbol{E}_{8}\right)$.

$W\left(\boldsymbol{E}_{8}\right)$ affects only the Wilson lines $\mu:=\sum_{i=1}^{8} \mu_{i} \omega_{i}$; it is clear that $\mu_{i}$ transforms in the same way as the simple root $\alpha_{i}$ of $\boldsymbol{E}_{8}$. The embedding $\imath$ of the finite root lattice $L\left(\boldsymbol{E}_{8}\right)$ in the Euclidean space $\mathbf{R}^{8}$ defined by

$$
\imath:\left(\begin{array}{c}
\alpha_{1} \\
\alpha_{2} \\
\alpha_{3} \\
\alpha_{4} \\
\alpha_{5} \\
\alpha_{6} \\
\alpha_{7} \\
\alpha_{8}
\end{array}\right) \rightarrow\left(\begin{array}{rrrrrrrr}
\frac{1}{2} & -\frac{1}{2} & -\frac{1}{2} & -\frac{1}{2} & -\frac{1}{2} & -\frac{1}{2} & -\frac{1}{2} & \frac{1}{2} \\
-1 & 1 & 0 & 0 & 0 & 0 & 0 & 0 \\
0 & -1 & 1 & 0 & 0 & 0 & 0 & 0 \\
0 & 0 & -1 & 1 & 0 & 0 & 0 & 0 \\
0 & 0 & 0 & -1 & 1 & 0 & 0 & 0 \\
0 & 0 & 0 & 0 & -1 & 1 & 0 & 0 \\
0 & 0 & 0 & 0 & 0 & -1 & 1 & 0 \\
1 & 1 & 0 & 0 & 0 & 0 & 0 & 0
\end{array}\right)\left(\begin{array}{l}
\mathbf{e}_{1} \\
\mathbf{e}_{2} \\
\mathbf{e}_{3} \\
\mathbf{e}_{4} \\
\mathbf{e}_{5} \\
\mathbf{e}_{6} \\
\mathbf{e}_{7} \\
\mathbf{e}_{8}
\end{array}\right)
$$


where $\left\{\mathbf{e}_{i}\right\}$ is an orthonormal basis of $\mathbf{R}^{8}$, greatly simplifies the description of $W\left(\boldsymbol{E}_{8}\right)$; it is generated by (i) the permutations of $\left\{\mathbf{e}_{i}\right\}$, (ii) the sign flips of even number of $\mathbf{e}_{i} \mathrm{~s}$ and (iii) the involution, which is the Weyl reflection with respect to the root $\theta-\sum_{i=1}^{7} \alpha_{i}$, $\mathbf{e}_{i} \mapsto \mathbf{e}_{i}-1 / 4 \sum_{j=1}^{8} \mathbf{e}_{j}[10,12]$. If $\imath(\mu)=\sum_{i=1}^{8} m_{i}(\mu) \mathbf{e}_{i}$, we call $\left(m_{i}(\mu)\right)$ the Euclidean coordinates of $\mu$.

On the other hand, the translation $t_{\beta}$ by $\beta \in L\left(\boldsymbol{E}_{8}\right)$ is given by

$$
t_{\beta}(x)=x+(x \mid \delta) \beta-\left\{\frac{(\beta \mid \beta)}{2}(x \mid \delta)+(x \mid \beta)\right\} \delta .
$$

Substituting $x=J(2.5)$, we see the $t_{\beta}$ action on the Kähler moduli parameters:

$$
\sigma \mapsto \sigma+\frac{1}{2}(\beta \mid \beta) \tau+(\mu \mid \beta), \quad \tau \mapsto \tau, \quad \mu \mapsto \mu+\tau \beta
$$

which is familiar as a symmetry in classical theta functions. Note that to realize another symmetry translation $\sigma \rightarrow \sigma, \tau \rightarrow \tau, \mu \rightarrow \mu+\alpha$, as a Weyl group action, we need to

consider the doubly-affinized $\boldsymbol{E}_{8}$ algebra, $\boldsymbol{E}_{9}^{(1)}[8,33,35]$, which might be possible only if we extend $H_{2}\left(B_{9}\right)$ to the full homology lattice $H_{0}\left(B_{9}\right) \oplus H_{2}\left(B_{9}\right) \oplus H_{4}\left(B_{9}\right)$.

\subsection{Partition functions}

The Gromov-Witten partition function $Z_{g ; n}(\tau \mid \mu)$ of genus $g$ and winding number $n$ of the local $B_{9}$ model with the Kähler moduli $J$ given in (2.5) is defined by the expansion coefficient of the genus $g$ potential $F_{g}[3]$ :

$$
F_{g}(\sigma, \tau, \mu)=\sum_{n=1}^{\infty} p^{n} Z_{g ; n}(\tau \mid \mu), \quad p:=\mathrm{e}^{2 \pi \mathrm{i} \sigma} .
$$

We remark here that the genus $g$ refers to that of type IIA string, while the winding number $n$ refers to that of E-string.

Using $\varphi(\tau):=\prod_{n=1}^{\infty}\left(1-q^{n}\right)$, with $q=\mathrm{e}^{2 \pi \mathrm{i} \tau}$, we can write $Z_{g ; n}$ as

$$
Z_{g ; n}(\tau \mid \mu)=\frac{T_{g ; n}(\tau \mid \mu)}{\varphi(\tau)^{12 n}} .
$$

The numerator $T_{g ; n}$ is so-called a Weyl-invariant $\boldsymbol{E}_{8}$ quasi-Jacobi form [22] of index $n$ and weight $2 g-2+6 n$, which means that $T_{g ; n}(\tau \mid \mu)$ is invariant under the $W\left(\boldsymbol{E}_{8}\right)$ action on 
$\mu$, and it has the following transformation properties:

$$
\begin{aligned}
T_{g ; n}(\tau \mid \mu+\alpha+\beta \tau) & =\mathrm{e}^{-\pi \mathrm{i} n[(\beta \mid \beta) \tau+2(\beta \mid \mu)]} T_{g ; n}(\tau \mid \mu), \alpha, \beta \in L\left(\boldsymbol{E}_{8}\right), \\
\hat{T}_{g ; n}\left(\frac{a \tau+b}{c \tau+d} \mid \frac{\mu}{c \tau+d}\right) & =(c \tau+d)^{2 g-2+6 n} \mathrm{e}^{\frac{n \pi \mathrm{i} c}{c \tau+d}(\mu \mid \mu)} \hat{T}_{g ; n}(\tau \mid \mu), \\
\left(\begin{array}{ll}
a & b \\
c & d
\end{array}\right) & \in S L(2 ; \mathbf{Z}),
\end{aligned}
$$

where $\hat{T}_{g ; n}$ is obtained from $T_{g ; n}$ by replacement of each $E_{2}(\tau)$ in it with $\hat{E}_{2}(\tau):=E_{2}(\tau)-$ $3 /(\pi \operatorname{Im} \tau)$, which gets rid of the modular anomaly of $Z_{g ; n}$ which comes from

$$
E_{2}\left(\frac{a \tau+b}{c \tau+d}\right)=(c \tau+d)^{2}\left(E_{2}(\tau)+\frac{12}{2 \pi \mathrm{i}} \frac{c}{c \tau+d}\right)
$$

at the sacrifice of holomorphy [36]. (2.11) shows that $\mathrm{e}^{2 \pi \mathrm{i} n \sigma} T_{g ; n}(\tau \mid \mu)$ is invariant under the translation $t_{\beta}$ (2.8) as expected. In particular, the genus zero, singly winding partition function can be given by the classical level one $\boldsymbol{E}_{8}$ theta function $[23,7,27]$ :

$$
T_{0 ; 1}(\tau \mid \mu)=\Theta_{\boldsymbol{E}_{8}}(\tau \mid \mu):=\sum_{\alpha \in L\left(\boldsymbol{E}_{8}\right)} \mathrm{e}^{\pi \mathrm{i}(\alpha \mid \alpha) \tau+2 \pi \mathrm{i}(\alpha \mid \mu)}
$$

$\Theta_{\boldsymbol{E}_{8}}$ can be written in terms of the Euclidean coordinates for the Wilson lines as

$$
\Theta_{\boldsymbol{E}_{8}}(\tau \mid \mu)=\frac{1}{2} \sum_{a=1}^{4} \prod_{i=1}^{8} \vartheta_{a}\left(\tau \mid m_{i}\right), \quad \iota(\mu)=\sum_{i=1}^{8} m_{i} \mathbf{e}_{i}
$$

The relation to curve counting problem is as follows [18]: in $Z_{0 ; 1}(\tau \mid \mu)$, the $\boldsymbol{E}_{8}$ theta function part is regarded as the contribution to $Z_{0 ; 1}$ from the Mordell-Weil lattice [9], while the denominator part $\varphi^{12}[23,7]$ from the twelve degenerate elliptic fibres of the fibration $\pi: B_{9} \rightarrow \mathbf{P}^{1}$.

Furthermore it is clear from the analysis of the BPS states in $[18,19]$ that higher genus partition functions of the singly winding sector can be obtained by

$$
\Phi_{1}:=\sum_{g=0}^{\infty} Z_{g ; 1}(\tau \mid \mu) x^{2 g-2}=\left(\frac{\eta(\tau)^{3}}{\vartheta_{1}\left(\tau \mid \frac{x}{2 \pi}\right)}\right)^{2} Z_{0 ; 1}(\tau \mid \mu) .
$$

The genus expansion of the right hand side reads [36],

$$
\left(\frac{\eta(\tau)^{3}}{\vartheta_{1}\left(\tau \mid \frac{x}{2 \pi}\right)}\right)^{2}=\frac{1}{x^{2}} \exp \left[\sum_{k=1}^{\infty} \frac{(-1)^{k+1} b_{2 k}}{k(2 k) !} E_{2 k}(\tau) x^{2 k}\right]=\sum_{g=0}^{\infty} x^{2 g-2} A_{g}(\tau),
$$


where $b_{n}$ and the $(2 k)$ th Eisenstein series $E_{2 k}(\tau)$ are defined by

$$
\frac{x}{\mathrm{e}^{x}-1}=\sum_{n=0}^{\infty} b_{n} \frac{x^{n}}{n !}, \quad E_{2 k}(\tau)=1-\frac{4 k}{b_{2 k}} \sum_{n=1}^{\infty} \sigma_{2 k-1}(n) q^{n}, \quad \sigma_{k}(n):=\sum_{m \mid n} m^{k} .
$$

As $E_{2 k} \in \mathbf{Q}\left[E_{4}, E_{6}\right]$, that is, $E_{8}=E_{4}^{2}, E_{10}=E_{4} E_{6}, E_{12}=1 / 691\left(250 E_{6}^{2}+441 E_{4}^{3}\right), E_{14}=E_{4}^{2} E_{6}$, for example, the coefficients $A_{g}$ above can be reduced to

$$
\begin{aligned}
& A_{1}=\frac{1}{12} E_{2}, A_{2}=\frac{1}{1440}\left(E_{4}+5 E_{2}^{2}\right), A_{3}=\frac{1}{362880}\left(4 E_{6}+21 E_{2} E_{4}+35 E_{2}^{3}\right), \\
& A_{4}=\frac{1}{87091200}\left(39 E_{4}^{2}+80 E_{2} E_{6}+210 E_{2}^{2} E_{4}+175 E_{2}^{4}\right), \\
& A_{5}=\frac{1}{11496038400}\left(136 E_{4} E_{6}+429 E_{2} E_{4}^{2}+440 E_{2}^{2} E_{6}+770 E_{2}^{3} E_{4}+385 E_{2}^{5}\right), \ldots
\end{aligned}
$$

The partition functions $Z_{g ; n}$ obeys the modular anomaly equation

$$
\begin{aligned}
\frac{\partial Z_{g ; n}(\tau \mid \mu)}{\partial E_{2}(\tau)} & =\frac{1}{24} \sum_{h+h^{\prime}=g} \sum_{k+k^{\prime}=n} k k^{\prime} Z_{h ; k}(\tau \mid \mu) Z_{h^{\prime} ; k^{\prime}}(\tau \mid \mu) \\
& +\frac{1}{24} n(n+1) Z_{g-1 ; n}(\tau \mid \mu),
\end{aligned}
$$

which has first been found in genus zero partition functions [29], and then generalized to higher genus partition functions in [18]. This can be rewritten using the genus $g$ potential (2.9) with each $E_{2}(\tau)$ in it replaced by $\hat{E}_{2}(\tau), \hat{F}_{g}=\sum_{n=1}^{\infty} p^{n} \hat{Z}_{g ; n}$, as

$$
-16 \pi \mathrm{i}(\operatorname{Im} \tau)^{2}\left(\frac{\partial \hat{F}_{g}}{\partial \bar{\tau}}\right)=\sum_{h=0}^{g} \Theta_{p} \hat{F}_{h} \Theta_{p} \hat{F}_{g-h}+\left(\Theta_{p}+1\right) \Theta_{p} \hat{F}_{g-1},
$$

where $\Theta_{p}=p \partial / \partial p$ is the Euler derivative. Compare this with the holomorphic anomaly equation for the topological string amplitude $F_{g}$ for $g>1$ on a Calabi-Yau threefold $X$ expressed in a general coordinate system [3]

$$
\partial_{\bar{i}} \hat{F}_{g}=\frac{1}{2} C_{\bar{i} \bar{j} \bar{k}} \mathrm{e}^{2 K} G^{j \bar{j}} G^{k \bar{k}}\left(\sum_{h=1}^{g-1} D_{j} \hat{F}_{h} D_{k} \hat{F}_{g-h}+D_{j} D_{k} \hat{F}_{g-1}\right) .
$$

where $G_{i \bar{j}}=\partial_{i} \partial_{\bar{j}} K$ is the Weil-Petersson-Zamolodchikov metric on the complex moduli space $\mathcal{M}\left(X^{*}\right)$ of the mirror $X^{*}, \mathrm{e}^{-K}$ a natural Hermite metric on a positive line bundle $L \rightarrow \mathcal{M}\left(X^{*}\right)$, with the holomorphic three-form of $X^{*}$ being a local section, and $C_{i j k}$ the $27^{3}$ Yukawa coupling. The covariant derivatives above have contributions not only from 
the Levi-Civita connection $\Gamma_{i j}^{k}=G^{k \bar{l}} \partial_{j} G_{i \bar{l}}$ but also from the Hermite connection $-\partial_{i} K$ on $L$ because $\hat{F}_{g}$ is a section of $L^{2 g-2}$,

$$
D_{i} \hat{F}_{g}=\left[\partial_{i}+(2-2 g) \partial_{i} K\right] \hat{F}_{g}, \quad D_{i} D_{j} \hat{F}_{g}=\left[\partial_{i}+(2-2 g) \partial_{i} K\right] D_{j} \hat{F}_{g}-\Gamma_{i j}^{k} D_{k} \hat{F}_{g} .
$$

Even more important is the potential $\Phi_{n}$ of fixed winding number $n$ :

$$
\Phi_{n}(\lambda ; \tau, \mu)=\sum_{g=0}^{\infty} x^{2 g-2} Z_{g ; n}(\tau \mid \mu), \quad \lambda:=\mathrm{e}^{\frac{1}{12} E_{2}(\tau) x^{2}} .
$$

Introduction of $\lambda$ and the full potential $\mathcal{A}:=\sum_{g=0}^{\infty} x^{2 g-2} F_{g}=\sum_{n=1}^{\infty} p^{n} \Phi_{n}$ greatly simplifies the modular anomaly equation (2.17):

$$
\Theta_{\lambda} \exp (\mathcal{A})=\frac{1}{2} \Theta_{p}\left(\Theta_{p}+1\right) \exp (\mathcal{A}) .
$$

If we substitute the $p$-expansion $\exp (\mathcal{A})=\sum_{n=0}^{\infty} p^{n} \psi_{n}\left(\Phi_{1}, \ldots, \Phi_{n}\right)$, where $\psi_{n}$ is the $n$th Schur polynomial, then we obtain $\Theta_{\lambda} \psi_{n}=n(n+1) / 2 \psi_{n}$, hence

$$
\psi_{n}\left(\Phi_{1}, \ldots, \Phi_{n}\right)=\lambda^{\frac{1}{2} n(n+1)} \psi_{n}\left(\Phi_{1}^{0}, \ldots, \Phi_{n}^{0}\right)
$$

where $\Phi_{n}^{0}=\left.\Phi_{n}\right|_{E_{2}=0}$ is the anomaly-free part; in particular, $\Phi_{1}=\lambda \Phi_{1}^{0}$ from (2.16).

Finally (2.20) enables us to express the solution for the modular anomaly equation (2.17) concisely by $\Phi_{n}$ with the anomaly-free part $\Phi_{n}^{0}$ as its integration constant:

$$
\begin{aligned}
\Phi_{2} & =\frac{1}{2}(\lambda-1)\left(\Phi_{1}\right)^{2}+\lambda^{3} \Phi_{2}^{0}, \\
\Phi_{3} & =-\frac{1}{6}(\lambda-1)^{2}(2 \lambda+1)\left(\Phi_{1}\right)^{3}+\left(\lambda^{2}-1\right) \Phi_{1} \Phi_{2}+\lambda^{6} \Phi_{3}^{0}, \\
\Phi_{4} & =\frac{1}{24}(\lambda-1)^{3}\left(6 \lambda^{3}+6 \lambda^{2}+3 \lambda+1\right)\left(\Phi_{1}\right)^{4}+\frac{1}{2}\left(\lambda^{4}-1\right)\left(\Phi_{2}\right)^{2}+\left(\lambda^{3}-1\right) \Phi_{1} \Phi_{3} \\
& -\frac{1}{2}(\lambda-1)^{2}(\lambda+1)\left(2 \lambda^{2}+\lambda+1\right)\left(\Phi_{1}\right)^{2} \Phi_{2}+\lambda^{10} \Phi_{4}^{0} .
\end{aligned}
$$

We are interested in $\Phi_{n}$ also because it encapsulates the interaction of $n$ E-strings, which can be quite different from that of fundamental strings [27].

We also give the prediction of the leading term of the partition function:

$$
Z_{g ; n}(\tau \mid \mu)=\beta_{g, 0} n^{2 g-3}+O\left(q^{n}\right), \quad \beta_{g, 0}:=\frac{\left|b_{2 g}(2 g-1)\right|}{(2 g) !},
$$

which generalizes the genus zero result in [29]. This can be used to partially fix the integration constants of the partition function. 


\section{Four Torus Models}

In this section, we investigate the instanton expansion by means of elliptic modular forms [37] of the periods of four one-parameter families of elliptic curves, $\boldsymbol{E}_{N}, N=5,6,7$, 8, each of which is defined as a complete intersection in a toric variety as shown in the Table 1. The results of this section will play an essential role in the instanton expansion of E-string model in the next section.

Table 1: Toric models

\begin{tabular}{|l|cc|}
\hline elliptic curve & \multicolumn{2}{|c|}{$\boldsymbol{E}_{9}$ del Pezzo } \\
\hline $\boldsymbol{E}_{5}: \mathbf{P}^{3}[2,2]$ & $\boldsymbol{E}_{5}$ & $\boldsymbol{E}_{\tilde{1}}$ \\
\hline $\boldsymbol{E}_{6}: \mathbf{P}^{2}[3]$ & $\boldsymbol{E}_{6}$ & $\boldsymbol{E}_{0}$ \\
\hline $\boldsymbol{E}_{7}: \mathbf{P}_{1,1,2}[4]$ & $\boldsymbol{E}_{7}$ & \\
\hline $\boldsymbol{E}_{8}:$ & $\mathbf{P}_{1,2,3}[6]$ & $\boldsymbol{E}_{8}$ \\
\hline
\end{tabular}

The $\boldsymbol{E}_{N}$ torus model have been studied in connection with the one-parameter families of the local $\boldsymbol{E}_{N}$ del Pezzo models [23, 24, 6, 31, 32], and Calabi-Yau threefolds with these elliptic fibers have been used to describe $4 \mathrm{D}$ string models the $\boldsymbol{E}_{8}$ gauge symmetry of which is broken to $\boldsymbol{E}_{N}$ by Wilson lines [1, 23].

\subsection{Periods of elliptic curves}

The Picard-Fuchs operator for the periods of the $\boldsymbol{E}_{N}$ family of elliptic curves is given by

$$
\mathcal{D}_{\text {ell }}^{(N)}=\Theta^{2}-z\left(\Theta+\alpha^{(N)}\right)\left(\Theta+\beta^{(N)}\right)
$$

where $\Theta=z \mathrm{~d} / \mathrm{d} z$ is the Euler derivative with respect to the bare modulus $z$, and $\alpha, \beta$ is defined by $\alpha+\beta=1$, and $\left(\alpha^{(5)}, \alpha^{(6)}, \alpha^{(7)}, \alpha^{(8)}\right)=(1 / 2,1 / 3,1 / 4,1 / 6)$.

The Gauss hypergeometric equation defined by (3.1) has the regular singular points at $z=0,1, \infty$. The solutions around $z=0$, which corresponds to the large radius limit point of the sigma model with the torus as target, are given by

$$
\varpi(z)=\sum_{n=0}^{\infty} a(n) z^{n}={ }_{2} F_{1}(\alpha, \beta ; 1 ; z),
$$




$$
\varpi_{D}(z)=\varpi(z) \log \left(\frac{z}{\kappa}\right)+\sum_{n=1}^{\infty} a(n) b(n) z^{n},
$$

where $\left(\kappa^{(5)}, \kappa^{(6)}, \kappa^{(7)}, \kappa^{(8)}\right)=(16,27,64,432)$, and

$$
a(n)=\frac{(\alpha)_{n}(\beta)_{n}}{(n !)^{2}}, \quad b(n)=\sum_{k=0}^{n-1}\left(\frac{1}{k+\alpha}+\frac{1}{k+\beta}-\frac{2}{k+1}\right),
$$

with $(\alpha)_{n}:=\Gamma(n+\alpha) / \Gamma(\alpha)$ the Pochhammer symbol.

The mirror map of the torus is defined by

$$
2 \pi \mathrm{i} \tau=\frac{\varpi_{D}(z)}{\varpi(z)}
$$

where $\tau$ is the Kähler parameter of the torus.

In terms of the local coordinate $u=1-z$ at another regular singular point $z=1$, the Picard-Fuchs operator takes the same form as one at $z=0$ (3.1), and the continuation of the solutions above becomes [32]

$$
\left(\begin{array}{c}
\varpi(z) \\
\varpi_{D}(z)
\end{array}\right) \rightarrow-\left(\begin{array}{cc}
0 & x \\
x^{-1} & 0
\end{array}\right)\left(\begin{array}{c}
\varpi(u) \\
\varpi_{D}(u)
\end{array}\right), \quad x=\frac{\sin (\pi \alpha)}{\pi} .
$$

Let $M_{0,1, \infty}$ be the monodromy matrices around the regular singular points $z=0,1, \infty$ with respect to the basis $\left\{\varpi_{D}(z) /(2 \pi \mathrm{i}), \varpi(z)\right\}$. We can compute $M_{0}$ and $M_{1}$ from $(3.2)$, (3.3) and (3.5) respectively:

$$
M_{0}=T=\left(\begin{array}{ll}
1 & 1 \\
0 & 1
\end{array}\right), \quad M_{1}=-S T^{(9-N)} S=\left(\begin{array}{cc}
1 & 0 \\
-(9-N) & 1
\end{array}\right),
$$

where $S$ and $T$ are the standard generators of $S L(2 ; \mathbf{Z})$. The remaining one $M_{\infty}$ can be obtained from $M_{\infty}=M_{1} M_{0}$. The monodromy group $\Gamma_{\text {ell }}$ is generated by $M_{0}$ and $M_{1}$; in particular, for $N \neq 8, \Gamma_{\text {ell }}^{(N)} \cong \Gamma_{0}(9-N)$, which is the Hecke subgroup of $S L(2 ; \mathbf{Z})[37]$

$$
\Gamma_{0}(h):=\left\{\left(\begin{array}{ll}
a & b \\
c & d
\end{array}\right) \in S L(2 ; \mathbf{Z}) \mid c \equiv 0 \bmod h\right\} .
$$

The structure of $M_{*}\left(\Gamma_{0}(9-N)\right)$, the graded ring of of the modular forms of even degree of $\Gamma_{0}(9-N)$, should be clear from

$$
\begin{array}{ll}
\boldsymbol{E}_{5}: & M_{*}\left(\Gamma_{0}(4)\right)=\mathbf{C}\left[\vartheta_{3}(2 \tau)^{4}, \vartheta_{4}(2 \tau)^{4}\right], \\
\boldsymbol{E}_{6}: & M_{*}\left(\Gamma_{0}(3)\right)=\left.\mathbf{C}\left[\varpi^{(6)}, H\right]\right|_{\text {even }}, \quad H:=\frac{\eta(\tau)^{9}}{\eta(3 \tau)^{3}}, \\
\boldsymbol{E}_{7}: & M_{*}\left(\Gamma_{0}(2)\right)=\mathbf{C}[A, B], \quad A:=\frac{1}{2}\left(\vartheta_{3}^{4}+\vartheta_{4}^{4}\right)(\tau), \quad B:=\vartheta_{3}^{4} \vartheta_{4}^{4}(\tau) .
\end{array}
$$


The fundamental solution $\varpi(z)$ admits the following expressions:

$$
\begin{array}{ll}
\varpi^{(5)}(z)=\Theta_{\boldsymbol{A}_{1} \oplus \boldsymbol{A}_{1}}(\tau) & =\vartheta_{3}(2 \tau)^{2}, \\
\varpi^{(6)}(z)=\Theta_{\boldsymbol{A}_{2}}(\tau) & =\vartheta_{3}(2 \tau) \vartheta_{3}(6 \tau)+\vartheta_{2}(2 \tau) \vartheta_{2}(6 \tau), \\
\varpi^{(7)}(z)^{2}=\Theta_{\boldsymbol{D}_{4}}(\tau) & =A(\tau), \\
\varpi^{(8)}(z)^{4}=\Theta_{\boldsymbol{E}_{8}}(\tau) & =E_{4}(\tau),
\end{array}
$$

where $\Theta_{\boldsymbol{K}}(\tau)$ is the theta function associated with the root lattice of $\boldsymbol{K}$.

Despite of the modular anomaly $(2.13), h E_{2}(h \tau)-E_{2}(\tau)$ is a sound modular form of $\Gamma_{0}(h)$ for each $h \in \mathbf{N}$. The following identity then shows that $\varpi(z)^{2}$ is an element of $M_{2}\left(\Gamma_{\text {ell }}^{(N)}\right)$ for $N=5,6,7$ :

$$
(8-N) \omega^{(N)}(z)^{2}=(9-N) E_{2}((9-N) \tau)-E_{2}(\tau) .
$$

Let us define for later use the modular function $e_{2 k}$ by $e_{2 k}(\tau)=E_{2 k}(\tau) \varpi^{-2 k}$ for each model, which turn out to be written in terms of $y:=1 /(1-z)$ :

$$
\begin{array}{lll}
\boldsymbol{E}_{5}: & e_{4}(\tau)=16-\frac{16}{y}+\frac{1}{y^{2}}, & e_{6}(\tau)=-64+\frac{96}{y}-\frac{30}{y^{2}}-\frac{1}{y^{3}}, \\
\boldsymbol{E}_{6}: & e_{4}(\tau)=9-\frac{8}{y}, & e_{6}(\tau)=-27+\frac{36}{y}-\frac{8}{y^{2}}, \\
\boldsymbol{E}_{7}: & e_{4}(\tau)=4-\frac{3}{y}, & e_{6}(\tau)=-8+\frac{9}{y}, \\
\boldsymbol{E}_{8}: & e_{4}(\tau)=1, & e_{6}(\tau)=-1+\frac{2}{y} .
\end{array}
$$

We see that $e_{4}$ and $e_{6}$ are subject to the algebraic relation:

$$
\begin{array}{ll}
\boldsymbol{E}_{5}: & 0=e_{4}^{3}-e_{6}^{2}-108 e_{4} e_{6}-8640 e_{6}-1620 e_{4}^{2}-17280 e_{4}+27648, \\
\boldsymbol{E}_{6}: & 0=8 e_{6}+18 e_{4}+e_{4}^{2}-27 \\
\boldsymbol{E}_{7}: & 0=3 e_{4}+e_{6}-4 .
\end{array}
$$

Let $T^{(N)}(\tau)$ be the modular function of $\Gamma_{\text {ell }}^{(N)}$ defined by

$$
\begin{aligned}
& T^{(5)}(\tau)=\left(\frac{\eta(\tau)}{\eta(4 \tau)}\right)^{8}, T^{(6)}(\tau)=\left(\frac{\eta(\tau)}{\eta(3 \tau)}\right)^{12}, T^{(7)}(\tau)=\left(\frac{\eta(\tau)}{\eta(2 \tau)}\right)^{24}, \\
& T^{(8)}(\tau)=\left(\frac{E_{4}(\tau)^{\frac{3}{2}}+E_{6}(\tau)}{2 \eta(\tau)^{12}}\right)^{2},
\end{aligned}
$$


where $\eta(\tau):=q^{1 / 24} \prod_{n=1}^{\infty}\left(1-q^{n}\right)$ is the Dedekind eta function with $q=\mathrm{e}^{2 \pi \mathrm{i} \tau}$. Then the inversion of the mirror map (3.4) can be given by

$$
\frac{1}{z(\tau)}=1+\frac{1}{\kappa} T(\tau)
$$

We note here that when $N=5,6,7, \Gamma_{\text {ell }}^{(N)}$ is a genus zero modular group, that is, the upper half plane divided by the action of $\Gamma_{\text {ell }}^{(N)}$ is completed to $\mathbf{P}^{1}$, and the $q$-expansion of $T^{(N)}(\tau)$ reproduces the Thompson series associated with $\Gamma_{\mathrm{ell}}^{(N)}[25]$.

Another useful expressions of $1-z$ by modular forms are

$$
\begin{array}{lll}
\boldsymbol{E}_{5}: 1-z(\tau)=\left(\frac{\vartheta_{4}(2 \tau)}{\vartheta_{3}(2 \tau)}\right)^{4}, & \boldsymbol{E}_{6}: 1-z(\tau)=\frac{H}{\varpi^{3}}, \\
\boldsymbol{E}_{7}: 1-z(\tau)=\frac{B}{A^{2}}, & \boldsymbol{E}_{8}: 1-z(\tau)=\frac{1}{2}\left(1+E_{6} E_{4}^{-\frac{3}{2}}(\tau)\right) .
\end{array}
$$

For each model, $\varpi(z)$ and $z$ satisfy the following equations:

$$
\frac{1}{2 \pi \mathrm{i}} \frac{\mathrm{d} z}{\mathrm{~d} \tau}=z(1-z) \varpi(z)^{2}, \quad \kappa \eta(\tau)^{24}=z(1-z)^{9-N} \varpi(z)^{12} .
$$

\subsection{Modular identities}

The following power series will play an important role in the instanton expansion of the E-string models:

$$
\begin{aligned}
\xi^{(0)}(z) & =\sum_{n=1}^{\infty} a^{(6)}(n) h(3 n) z^{n} \\
\xi^{(\tilde{1})}(z) & =\sum_{n=1}^{\infty} a^{(5)}(n) h(2 n) z^{n}, \\
\xi^{(N)}(z) & =\sum_{n=1}^{\infty} a^{(N)}(n) h(n) z^{n}, \quad \text { for } N=5,6,7,8 .
\end{aligned}
$$

where $h(n)=\sum_{k=1}^{n} k^{-1}$ is the harmonic function. A computer experiment gives the identities:

$$
\begin{array}{ll}
\boldsymbol{E}_{6}: & \psi^{(0)}(\tau)=\exp \left(-\frac{\xi^{(0)}(z)}{\varpi(z)}\right)=\left(\frac{27 q}{z}\right)^{\frac{1}{6}}(1-z)^{\frac{1}{2}} \\
\boldsymbol{E}_{5}: & \psi^{(\tilde{1})}(\tau)=\exp \left(-\frac{\xi^{(\tilde{1})}(z)}{\varpi(z)}\right)=\left(\frac{16 q}{z}\right)^{\frac{1}{4}}(1-z)^{\frac{1}{2}}, \\
\boldsymbol{E}_{N}: & \psi^{(N)}(\tau)=\exp \left(-\frac{\xi^{(N)}(z)}{\varpi(z)}\right)=\left(\frac{\kappa^{(N)} q}{z}\right)^{\frac{1}{2}}(1-z)^{\frac{1}{2}}=\left(q T^{(N)}(\tau)\right)^{\frac{1}{2}} .
\end{array}
$$




\section{Model Building of E-Strings}

In this section, we give six models: $\boldsymbol{E}_{N}, N=0, \tilde{1}, 5,6,7,8$, of $\boldsymbol{E}_{9}$ almost del Pezzo surface listed in Table 1, which is realized as a complete intersection in a toric variety, and analyze the Picard-Fuchs systems defined by them at the large radius point. Among the six models, we call the four $\boldsymbol{E}_{5,6,7,8}$ the principal series, the reason of which will become clear in the investigation of the Picard-Fuchs system of them.

\subsection{Principal series}

The $\boldsymbol{E}_{6,7,8}$ models are obtained as hypersurfaces in ambient toric threefolds with their Kähler classes inherited from those of the ambient spaces.

To describe these, let us first define the action of $\left(\mathbf{C}^{*}\right)^{2}$ on $\mathbf{C}^{5}$ for each model by

$$
\begin{aligned}
& \boldsymbol{E}_{6}: \quad\left(x_{1}, x_{2}, x_{3}, x_{4}, x_{5}\right) \rightarrow\left(\lambda x_{1}, \lambda x_{2}, \lambda^{-1} \mu x_{3}, \mu x_{4}, \mu x_{5}\right), \\
& \boldsymbol{E}_{7}: \quad\left(x_{1}, x_{2}, x_{3}, x_{4}, x_{5}\right) \rightarrow\left(\lambda x_{1}, \lambda x_{2}, \lambda^{-1} \mu x_{3}, \mu x_{4}, \mu^{2} x_{5}\right), \\
& \boldsymbol{E}_{8}: \quad\left(x_{1}, x_{2}, x_{3}, x_{4}, x_{5}\right) \rightarrow\left(\lambda x_{1}, \lambda x_{2}, \lambda^{-1} \mu x_{3}, \mu^{2} x_{4}, \mu^{3} x_{5}\right),
\end{aligned}
$$

where $\lambda, \mu \in \mathbf{C}^{*}$. Then the ambient toric variety $\boldsymbol{A}$ can be realized by the quotient $\boldsymbol{A}:=\left(\mathbf{C}^{5}-F\right) /\left(\mathbf{C}^{*}\right)^{2}$, where $F=\left\{x_{1}=x_{2}=0\right\} \cup\left\{x_{3}=x_{4}=x_{5}=0\right\}$ is the bad point set of the $\left(\mathbf{C}^{*}\right)^{2}$ action.

It is easy to see that the ambient space $\boldsymbol{A}$ for the $\boldsymbol{E}_{6,7,8}$ model has a structure of weighted projective surface bundle over $\mathbf{P}^{1}$ with fiber $\mathbf{P}^{2}, \mathbf{P}_{1,1,2}, \mathbf{P}_{1,2,3}$ respectively.

We can now define the $\boldsymbol{E}_{9}$ almost del Pezzo surface $B_{9}$ for the $\boldsymbol{E}_{6,7,8}$ model as a hypersurface in $\boldsymbol{A}$ of bidegree $(0,3),(0,4)$ and $(0,6)$ respectively, where the bidegree refers to the $(\lambda, \mu)$ charge of the defining polynomial. The Kähler classes for $B_{9}$ induced from those of the ambient space are given by

$$
\begin{aligned}
& J^{(6)}=\sigma[\delta]+\tau\left([\delta]+\mathcal{E}_{7}+\mathcal{E}_{8}+\mathcal{E}_{9}\right), \\
& J^{(7)}=\sigma[\delta]+\tau\left([\delta]+\mathcal{E}_{8}+\mathcal{E}_{9}\right), \\
& J^{(8)}=\sigma[\delta]+\tau\left([\delta]+\mathcal{E}_{9}\right)
\end{aligned}
$$

where we recall that $[\delta]$ is the first Chern class of $B_{9}$. The local model of $B_{9}$ embedded in a Calabi-Yau threefold is described by the total space of the canonical line bundle $K_{B_{9}}$, 
which is again realized as a hypersurface in the non-compact toric variety $\mathcal{O}_{A}(-1,0)$. From this fact, we can identify the Mori vectors of the local Calabi-Yau models, which plays an essential role in the formulation of mirror symmetry [16]:

$$
\begin{array}{lll}
\boldsymbol{E}_{6}: & l_{1}=(0 ; 1,1,-1,0,0,-1), & l_{2}=(-3 ; 0,0,1,1,1,0), \\
\boldsymbol{E}_{7}: & l_{1}=(0 ; 1,1,-1,0,0,-1), & l_{2}=(-4 ; 0,0,1,1,2,0), \\
\boldsymbol{E}_{8}: & l_{1}=(0 ; 1,1,-1,0,0,-1), & l_{2}=(-6 ; 0,0,1,2,3,0) .
\end{array}
$$

It will suffice here to point out that the first components of the vectors $l_{1}, l_{2}$ are (minus) the degrees of the hypersurface, while the rest the $\mathbf{C}^{*}$ charges of the homogeneous coordinates of $K_{\boldsymbol{A}}$, where the last one corresponds to the non-compact direction.

Next we consider the $\boldsymbol{E}_{5}$ model. The ambient toric variety in this case is a $\mathbf{P}^{3}$ bundle over $\mathbf{P}^{1}$. This space admits the quotient realization $\left(\mathbf{C}^{6}-F\right) /\left(\mathbf{C}^{*}\right)^{2}$, where the $\left(\mathbf{C}^{*}\right)^{2}$ action on $\mathbf{C}^{6}$ is defined by $\left(x_{1}, x_{2}, x_{3}, x_{4}, x_{5}, x_{6}\right) \rightarrow\left(\lambda x_{1}, \lambda x_{2}, \lambda^{-1} \mu x_{3}, \mu x_{4}, \mu x_{5}, \mu x_{6}\right)$, and the bad point set $F=\left\{x_{1}=x_{2}=0\right\} \cup\left\{x_{3}=x_{4}=x_{5}=x_{6}=0\right\}$.

The surface $B_{9}$ is defined by a complete intersection of two hypersurfaces of bidegree $(0,2)$. The Kähler class of the $\boldsymbol{E}_{5}$ model induced from the ambient space turns out to be

$$
J^{(5)}=\sigma[\delta]+\tau\left([\delta]+\mathcal{E}_{6}+\mathcal{E}_{7}+\mathcal{E}_{8}+\mathcal{E}_{9}\right) .
$$

The Mori vectors in this case can be seen as in the case of the $\boldsymbol{E}_{6,7,8}$ models above:

$$
\boldsymbol{E}_{5}: \quad l_{1}=(0,0 ; 1,1,-1,0,0,0,-1), \quad l_{2}=(-2,-2 ; 0,0,1,1,1,1,0) .
$$

\section{$4.2 \quad E_{0}$ and $E_{\tilde{1}}$ models}

We present here the remaining two models, $\boldsymbol{E}_{0}$ and $\boldsymbol{E}_{\tilde{1}}$. Because the ambient spaces for these models are products of projective spaces, rather than a twisted fiber bundle as in the case of principal series, the toric construction of them may be omitted.

The $\boldsymbol{E}_{0}$ model is realized as a hypersurface of bidegree $(1,3)$ in $\mathbf{P}^{1} \times \mathbf{P}^{2}$, while the $\boldsymbol{E}_{\tilde{1}}$ model as one of tridegree $(1,2,2)$ in $\mathbf{P}^{1} \times \mathbf{P}^{1} \times \mathbf{P}^{1}$.

The Kähler classes induced from the ambient spaces are given by

$$
\begin{aligned}
& J^{(0)}=\sigma[\delta]+\tau l, \\
& J^{(\tilde{1})}=\sigma[\delta]+\tau_{1}\left(l-\mathcal{E}_{1}\right)+\tau_{2}\left(l-\mathcal{E}_{2}\right) .
\end{aligned}
$$


Note that for the $\boldsymbol{E}_{\tilde{1}}$ model, we must put the restriction $\tau_{1}=\tau_{2}=\tau$ to obtain a twoparameter model.

The Mori vectors of these two models are

$$
\begin{array}{lll}
\boldsymbol{E}_{0}: & l_{1}=(-1 ; 1,1,0,0,0,-1), & l_{2}=(-3 ; 0,0,1,1,1,0), \\
\boldsymbol{E}_{\tilde{1}}: & l_{1}=(-1 ; 1,1,0,0,0,0,-1), & l_{2}=(-2 ; 0,0,1,1,0,0,0), \\
& l_{3}=(-2 ; 0,0,0,0,1,1,0) .
\end{array}
$$

\subsection{Kähler classes}

Each of the Kähler classes of the six models obtained above admits two important representations, the one of which reveals the blow-up/down scheme of the two-parameter family of $B_{9} \mathrm{~s}$, and the other the canonical form (2.5) suggested by the investigation of $6 \mathrm{D}$ E-string compactified on $\mathbf{T}^{2}[10,12]$ :

$$
\begin{aligned}
& J^{(0)}=\sigma c_{1}\left(B_{9}\right)+\frac{\tau}{3} c_{1}\left(B_{0}\right)=\left(\sigma+\frac{3}{2} \tau\right)[\delta]-3 \tau\left[\Lambda_{0}\right]-\tau\left[\omega_{8}\right], \\
& J^{(\tilde{1})}=\sigma c_{1}\left(B_{9}\right)+\frac{\tau}{2} c_{1}\left(B_{\tilde{1}}\right)=(\sigma+2 \tau)[\delta]-4 \tau\left[\Lambda_{0}\right]-\tau\left[\omega_{2}\right], \\
& J^{(5)}=\sigma c_{1}\left(B_{9}\right)+\tau c_{1}\left(B_{5}\right)=(\sigma+2 \tau)[\delta]-4 \tau\left[\Lambda_{0}\right]-\tau\left[\omega_{5}\right], \\
& J^{(6)}=\sigma c_{1}\left(B_{9}\right)+\tau c_{1}\left(B_{6}\right)=\left(\sigma+\frac{3}{2} \tau\right)[\delta]-3 \tau\left[\Lambda_{0}\right]-\tau\left[\omega_{6}\right], \\
& J^{(7)}=\sigma c_{1}\left(B_{9}\right)+\tau c_{1}\left(B_{7}\right)=(\sigma+1 \tau)[\delta]-2 \tau\left[\Lambda_{0}\right]-\tau\left[\omega_{7}\right], \\
& J^{(8)}=\sigma c_{1}\left(B_{9}\right)+\tau c_{1}\left(B_{8}\right)=\left(\sigma+\frac{1}{2} \tau\right)[\delta]-1 \tau\left[\Lambda_{0}\right],
\end{aligned}
$$

where we recall that $[\delta]=c_{1}\left(B_{9}\right)=3 l-\sum_{i=1}^{9} \mathcal{E}_{i}, \mathcal{E}_{9}=-\left[\Lambda_{0}\right]-1 / 2[\delta], B_{N}$ the $\boldsymbol{E}_{N}$ del Pezzo surface with $c_{1}\left(B_{N}\right)=3 l-\sum_{i=1}^{N} \mathcal{E}_{i}, B_{0}=\mathbf{P}^{2}$ with $c_{1}\left(B_{0}\right)=3 l$, and $B_{\tilde{1}}=\mathbf{P}^{1} \times \mathbf{P}^{1}$ with $c_{1}\left(B_{\tilde{1}}\right)=2\left(2 l-\mathcal{E}_{1}-\mathcal{E}_{2}\right)$.

The existence of the blow-down of each model to the one parameter family of the corresponding del Pezzo surface, where the name of the model comes, leads to the relation between Gromov-Witten invariants of these models as we shall see below. The appearance of a single fundamental weight in the Wilson line term in each model is also suggestive. 
There are a few words to be said on what should be the $E_{N}$ models for $N=1,2,3,4$. Their Kähler moduli are expected to be written as

$$
\begin{aligned}
& J^{(1)}=\sigma c_{1}\left(B_{9}\right)+\tau c_{1}\left(B_{1}\right)=(\sigma+4 \tau)[\delta]-8 \tau\left[\Lambda_{0}\right]-\tau\left(\left[\omega_{1}\right]+2\left[\omega_{8}\right]\right), \\
& J^{(2)}=\sigma c_{1}\left(B_{9}\right)+\tau c_{1}\left(B_{2}\right)=\left(\sigma+\frac{7}{2} \tau\right)[\delta]-7 \tau\left[\Lambda_{0}\right]-\tau\left(\left[\omega_{2}\right]+\left[\omega_{8}\right]\right), \\
& J^{(3)}=\sigma c_{1}\left(B_{9}\right)+\tau c_{1}\left(B_{3}\right)=(\sigma+3 \tau)[\delta]-6 \tau\left[\Lambda_{0}\right]-\tau\left[\omega_{3}\right], \\
& J^{(4)}=\sigma c_{1}\left(B_{9}\right)+\tau c_{1}\left(B_{4}\right)=\left(\sigma+\frac{5}{2} \tau\right)[\delta]-5 \tau\left[\Lambda_{0}\right]-\tau\left[\omega_{4}\right],
\end{aligned}
$$

In view of the fact that $B_{1,2,3}$ are themselves toric surfaces, we might expect that at least for $N=1,2,3, \boldsymbol{E}_{N}$ models can be realized as a hypersurface in the toric threefold $B_{N} \times \mathbf{P}^{1}$, which we will not pursue further.

\subsection{Periods}

The solutions to the Picard-Fuchs differential equations around the large radius limit point $\left(z_{1}, z_{2}\right)=(0,0)$ can be obtained by the Frobenius method [16]. We first define the formal power series $\Omega_{\rho}^{(N)}\left(z_{1}, z_{2}\right)$ from the Mori vectors $l_{1}, l_{2}$ :

$$
\Omega_{\rho}\left(z_{1}, z_{2}\right)=\sum_{n_{1}=0}^{\infty} \sum_{n_{2}=0}^{\infty} A\left(n_{1}+\rho_{1}, n_{2}+\rho_{2}\right) z_{1}^{n_{1}+\rho_{1}} z_{2}^{n_{2}+\rho_{2}}
$$

where $A\left(n_{1}, n_{2}\right)$ for the $\boldsymbol{E}_{0}$ and $\boldsymbol{E}_{\tilde{1}}$ models are

$$
\begin{aligned}
& A^{(0)}\left(n_{1}, n_{2}\right)=\left(\frac{1}{27}\right)^{n_{2}} \frac{\Gamma\left(1+n_{1}+3 n_{2}\right)}{\Gamma\left(1-n_{1}\right) \Gamma\left(1+n_{1}\right)^{2} \Gamma\left(1+n_{2}\right)^{3}}, \\
& A^{(\tilde{1})}\left(n_{1}, n_{2}\right)=\left(\frac{1}{16}\right)^{n_{2}} \frac{\Gamma\left(1+n_{1}+2 n_{2}\right) \Gamma\left(1+2 n_{2}\right)}{\Gamma\left(1-n_{1}\right) \Gamma\left(1+n_{1}\right)^{2} \Gamma\left(1+n_{2}\right)^{4}},
\end{aligned}
$$

while for the principal series $\boldsymbol{E}_{N}, N=5,6,7,8$,

$$
A^{(N)}\left(n_{1}, n_{2}\right):=\frac{\Gamma\left(\alpha+n_{2}\right) \Gamma\left(\beta+n_{2}\right)}{\Gamma(\alpha) \Gamma(\beta) \Gamma\left(1+n_{2}\right) \Gamma\left(1+n_{1}\right)^{2} \Gamma\left(1-n_{1}\right) \Gamma\left(1+n_{2}-n_{1}\right)} .
$$

We remark that the $\boldsymbol{E}_{\tilde{1}}$ model has been defined as the three parameter model. The three Mori vectors (4.15) produce the formal power series

$$
\Omega_{\rho}\left(z_{1}, w_{1}, w_{2}\right)=\sum_{n_{1}=0}^{\infty} \sum_{k_{1}=0}^{\infty} \sum_{k_{2}=0}^{\infty} A\left(n_{1}+\rho_{1}, k_{1}+\rho_{21}, k_{2}+\rho_{22}\right) z_{1}^{n_{1}+\rho_{1}} w_{1}^{k_{1}+\rho_{21}} w_{2}^{k_{2}+\rho_{22}},
$$




$$
A\left(n_{1}, k_{1}, k_{2}\right)=\frac{\Gamma\left(1+n_{1}+2 k_{1}+2 k_{2}\right)}{\Gamma\left(1-n_{1}\right) \Gamma\left(1+n_{1}\right)^{2} \Gamma\left(1+k_{1}\right)^{2} \Gamma\left(1+k_{2}\right)^{2}} .
$$

Then the reduction to the two parameter model is achieved by setting $w_{1}=w_{2}=z_{2} / 16$; correspondingly, the coefficient of the power series reduces to the one described in (4.28):

$$
\begin{aligned}
16^{n_{2}} A\left(n_{1}, n_{2}\right) & =\sum_{k_{1}+k_{2}=n_{2}} A\left(n_{1}, k_{1}, k_{2}\right) \\
& =\frac{\Gamma\left(1+n_{1}+2 n_{2}\right)}{\Gamma\left(1-n_{1}\right) \Gamma\left(1+n_{1}\right)^{2} \Gamma\left(1+n_{2}\right)^{2}} \sum_{k_{1}+k_{2}=n_{2}}\left(\frac{\left(n_{2}\right) !}{\left(k_{1}\right) !\left(k_{2}\right) !}\right)^{2} \\
& =\frac{\Gamma\left(1+n_{1}+2 n_{2}\right) \Gamma\left(1+2 n_{2}\right)}{\Gamma\left(1-n_{1}\right) \Gamma\left(1+n_{1}\right)^{2} \Gamma\left(1+n_{2}\right)^{4}} .
\end{aligned}
$$

The computation of the Picard-Fuchs operators can be done in a standard manner once we know the form of $A\left(n_{1}, n_{2}\right)[16]$. For $\boldsymbol{E}_{0}$ and $\boldsymbol{E}_{\tilde{1}}$ model we have

$$
\begin{aligned}
& \mathcal{D}_{1}^{(0)}=\Theta_{1}^{2}+z_{1} \Theta_{1}\left(\Theta_{1}+3 \Theta_{2}+1\right), \\
& \mathcal{D}_{2}^{(0)}=9 \Theta_{2}^{2}-3 \Theta_{1} \Theta_{2}-z_{2}\left(3 \Theta_{2}+\Theta_{1}+1\right)\left(3 \Theta_{2}+\Theta_{1}+2\right)-3 z_{1} \Theta_{1} \Theta_{2}, \\
& \mathcal{D}_{1}^{(1)}=\Theta_{1}^{2}+z_{1} \Theta_{1}\left(\Theta_{1}+2 \Theta_{2}+1\right), \\
& \mathcal{D}_{2}^{(1)}=4 \Theta_{2}^{2}-2 \Theta_{1} \Theta_{2}-z_{2}\left(2 \Theta_{2}+1\right)\left(2 \Theta_{2}+\Theta_{1}+1\right)-2 z_{1} \Theta_{1} \Theta_{2},
\end{aligned}
$$

and for the principal series $\boldsymbol{E}_{N}$ with $N=5,6,7,8$,

$$
\begin{aligned}
& \mathcal{D}_{1}^{(N)}=\Theta_{1}^{2}-z_{1} \Theta_{1}\left(\Theta_{1}-\Theta_{2}\right), \\
& \mathcal{D}_{2}^{(N)}=\Theta_{2}\left(\Theta_{2}-\Theta_{1}\right)-z_{2}\left(\Theta_{2}+\alpha^{(N)}\right)\left(\Theta_{2}+\beta^{(N)}\right),
\end{aligned}
$$

where $\Theta_{1}=z_{1} \partial_{z_{1}}$ and $\Theta_{2}=z_{2} \partial_{z_{2}}$. In passing, we remark here that the Picard-Fuchs system for the principal series gives an Appell-Horn hypergeometric system in four ways according to the choice of the base point $\left(z_{1}^{ \pm 1}=0, z_{2}^{ \pm 1}=0\right)$. It would be quite interesting to analyze their monodromies.

The Frobenius method then gives the four solutions of the Picard-Fuchs system (4.30), (4.31), (4.32):

$$
\varpi\left(z_{2}\right)=\left.\Omega_{\rho}\left(z_{1}, z_{2}\right)\right|_{\rho=0}
$$




$$
\begin{aligned}
\varpi_{D}\left(z_{2}\right) & =\left.\partial_{\rho_{2}} \Omega_{\rho}\left(z_{1}, z_{2}\right)\right|_{\rho=0}, \\
\phi\left(z_{1}, z_{2}\right) & =\left.\partial_{\rho_{1}} \Omega_{\rho}\left(z_{1}, z_{2}\right)\right|_{\rho=0}, \\
\phi_{D}^{(0)}\left(z_{1}, z_{2}\right) & =\left.\left(\partial_{\rho_{1}} \partial_{\rho_{2}}+\frac{1}{6} \partial_{\rho_{2}}^{2}\right) \Omega_{\rho}^{(0)}\left(z_{1}, z_{2}\right)\right|_{\rho=0}, \\
\phi_{D}^{(\tilde{1})}\left(z_{1}, z_{2}\right) & =\left.\left(\partial_{\rho_{1}} \partial_{\rho_{2}}+\frac{1}{4} \partial_{\rho_{2}}^{2}\right) \Omega_{\rho}^{(\tilde{1})}\left(z_{1}, z_{2}\right)\right|_{\rho=0}, \\
\phi_{D}^{(N)}\left(z_{1}, z_{2}\right) & =\left.\left(\partial_{\rho_{1}} \partial_{\rho_{2}}+\frac{1}{2} \partial_{\rho_{2}}^{2}\right) \Omega_{\rho}^{(N)}\left(z_{1}, z_{2}\right)\right|_{\rho=0}, \quad N=5,6,7,8 .
\end{aligned}
$$

The first two solutions $\varpi$ and $\varpi_{D}$ are the same as those of the corresponding torus (3.2), (3.3), which is evident from the structure of the Picard-Fuchs operators above.

The third one is given by

$$
\phi\left(z_{1}, z_{2}\right)=\varpi\left(z_{2}\right) \log \left(z_{1}\right)+\xi^{(N)}\left(z_{2}\right)+\sum_{n_{1}=1}^{\infty} \mathcal{L}_{n_{1}}^{(N)} \varpi\left(z_{2}\right) z_{1}^{n_{1}}
$$

where $\xi^{(N)}\left(z_{2}\right)$ has been defined in (3.20), (3.21) and (3.22) and $\mathcal{L}_{n}^{(N)}$ is the differential operator defined by

$$
\begin{aligned}
\mathcal{L}_{n}^{(0)} & =\frac{(-1)^{n}}{n \cdot n !} \prod_{k=1}^{n}\left(3 \Theta_{2}+k\right), \\
\mathcal{L}_{n}^{(\tilde{1})} & =\frac{(-1)^{n}}{n \cdot n !} \prod_{k=1}^{n}\left(2 \Theta_{2}+k\right), \\
\mathcal{L}_{n}^{(N)} & =\frac{(-1)^{n}}{n \cdot n !} \prod_{k=0}^{n-1}\left(\Theta_{2}-k\right), \quad N=5,6,7,8 .
\end{aligned}
$$

The flat coordinates $\tau, \sigma$ are obtained by the mirror map

$$
2 \pi \mathrm{i} \tau=\frac{\varpi_{D}\left(z_{2}\right)}{\varpi\left(z_{2}\right)}, \quad 2 \pi \mathrm{i} \sigma=\frac{\phi\left(z_{1}, z_{2}\right)}{\varpi\left(z_{2}\right)} .
$$

Let $c_{n}:=\mathcal{L}_{n} \varpi / \varpi$, then (4.43) with (3.23), (3.24), (3.25) yields

$$
\mathrm{e}^{2 \pi \mathrm{i} \sigma} \psi^{(N)}(\tau)=z_{1} \exp \left(\sum_{n_{1}=1}^{\infty} c_{n_{1}}(\tau) z_{1}^{n_{1}}\right),
$$

from which we know that the inversion of the mirror for $z_{1}$ takes the form

$$
z_{1}=\tilde{p}\left(1+\sum_{n=1}^{\infty} d_{n}(\tau) \tilde{p}^{n}\right)
$$


where $\tilde{p}:=\mathrm{e}^{2 \pi \mathrm{i} \sigma} \psi(\tau)$, and $d_{n} \in \mathbf{Q}\left[c_{1}, \ldots, c_{n}\right]$; explicitly,

$$
\begin{aligned}
& d_{1}=-c_{1}, \quad d_{2}=\frac{3}{2} c_{1}^{2}-c_{2}, \quad d_{3}=-\frac{8}{3} c_{1}^{3}+4 c_{1} c_{2}-c_{3}, \\
& d_{4}=\frac{125}{24} c_{1}^{4}-\frac{25}{2} c_{1}^{2} c_{2}+5 c_{1} c_{3}+\frac{5}{2} c_{2}^{2}-c_{4}, \ldots
\end{aligned}
$$

After some tedious calculations, we find that the last solution $\phi_{D}$ is given by

$$
\begin{aligned}
\phi_{D}^{(0)}\left(z_{1}, z_{2}\right) & =(2 \pi \mathrm{i})^{2} \varpi\left(z_{2}\right)\left(\sigma \tau+\frac{1}{6} \tau^{2}-\frac{1}{6}\right)-\sum_{n=1}^{\infty} \frac{f_{n}\left(z_{2}\right)}{\varpi\left(z_{2}\right)} z_{1}^{n}, \\
\phi_{D}^{(\tilde{1})}\left(z_{1}, z_{2}\right) & =(2 \pi \mathrm{i})^{2} \varpi\left(z_{2}\right)\left(\sigma \tau+\frac{1}{4} \tau^{2}-\frac{1}{8}\right)-\sum_{n=1}^{\infty} \frac{f_{n}\left(z_{2}\right)}{\varpi\left(z_{2}\right)} z_{1}^{n}, \\
\phi_{D}^{(N)}\left(z_{1}, z_{2}\right) & =(2 \pi \mathrm{i})^{2} \varpi\left(z_{2}\right)\left(\sigma \tau+\frac{1}{2} \tau^{2}-\frac{1}{2(9-N)}\right)-\sum_{n=1}^{\infty} \frac{f_{n}\left(z_{2}\right)}{\varpi\left(z_{2}\right)} z_{1}^{n},
\end{aligned}
$$

where $f_{n}\left(z_{2}\right)$ is the "higher Wronskian" defined by

$$
f_{n}\left(z_{2}\right)=-\left(\varpi \mathcal{L}_{n} \varpi_{D}-\varpi_{D} \mathcal{L}_{n} \varpi\right)\left(z_{2}\right)
$$

It must be noted here that a crucial ingredient in obtaining the concise expression for $\phi_{D}$ above is the following formula of combinatoric nature:

$$
\left(\sum_{n=1}^{\infty} a(n) k(n) z_{2}^{n}\right)\left(\sum_{n=0}^{\infty} a(n) z_{2}^{n}\right)=\left(\sum_{n=1}^{\infty} a(n) b(n) z_{2}^{n}\right)\left(\sum_{n=1}^{\infty} a(n) s(n) z_{2}^{n}\right),
$$

where $s(n)$ and $k(n)$ are defined by

$$
s(n)=\sum_{l=0}^{n-1}\left(\frac{1}{l+\alpha}+\frac{1}{l+\beta}\right), k(n)=s(n)\left(s(n)-2 \sum_{l=0}^{n-1} \frac{1}{l+1}\right)-\sum_{l=0}^{n-1}\left(\frac{1}{(l+\alpha)^{2}}+\frac{1}{(l+\beta)^{2}}\right) .
$$

The instanton part of the genus zero prepotential of the model $F_{0}$ is defined by

$$
\frac{1}{2 \pi \mathrm{i}}\left(\frac{\partial F_{0}}{\partial \sigma}\right)=h \sum_{n=1}^{\infty} \frac{f_{n}\left(z_{2}\right)}{\varpi\left(z_{2}\right)^{2}} z_{1}^{n}
$$

where $\left(h^{(0)}, h^{(\tilde{1})}, h^{(5)}, h^{(6)}, h^{(7)}, h^{(8)}\right)=(3,4,4,3,2,1)$ is the normalization factor, which may be found, for example, by computation of the classical central charge of a D-branes system corresponding to a coherent sheaf $\mathcal{F}$ with the Kähler class $J^{(N)}[31]$ :

$$
Z^{\text {class }}(\mathcal{F})=-\left[\exp \left(-J^{(N)}\right) \cdot \operatorname{ch}(\mathcal{F}) \cdot\left(\left[B_{9}\right]+\frac{1}{2}[\delta]+\frac{1}{2}[\mathrm{pt}]\right)\right]
$$




\subsection{Relation to Seiberg-Witten periods}

In this subsection we show how to realize the two periods $\phi$ and $\phi_{D}$, which have been obtained as the solutions of the Picard-Fuchs differential equations, as the Seiberg-Witten integrals of the 6D non-critical string theory [11] compactified on $\mathbf{T}^{2}$ to $4 \mathrm{D}$ [10, 12, 24, 29].

Let $\phi_{0}\left(z_{1}, z_{2}\right)$ be a solution of the Picard-Fuchs system (4.30), (4.31), or (4.32). It is easy to see that if $\Theta_{1} \phi_{0}=0$, then $\mathcal{D}_{1} \phi_{0}=0$ is automatic and $\mathcal{D}_{2} \phi_{0}=0$ reduces to the Picard-Fuchs equation for the corresponding elliptic curve: $\mathcal{D}_{\text {ell }} \phi_{0}=0$; hence $\phi_{0}$ is a linear combination of $\varpi$ and $\varpi_{D}$.

Consider the case $\Theta_{1} \phi_{0} \neq 0$. The first equation $\mathcal{D}_{1} \phi_{0}=0$ gives a constraint on the function form of $\Theta_{1} \phi_{0}$ :

$$
\begin{aligned}
\boldsymbol{E}_{0}: & \Theta_{1} \phi_{0}\left(z_{1}, z_{2}\right)=\frac{1}{1+z_{1}} \omega\left(\tilde{z}_{2}\right), & \tilde{z}_{2}:=\frac{z_{2}}{\left(1+z_{1}\right)^{3}}, \\
\boldsymbol{E}_{\tilde{1}}: & \Theta_{1} \phi_{0}\left(z_{1}, z_{2}\right)=\frac{1}{1+z_{1}} \omega\left(\tilde{z}_{2}\right), & \tilde{z}_{2}:=\frac{z_{2}}{\left(1+z_{1}\right)^{2}}, \\
\boldsymbol{E}_{5,6,7,8}: & \Theta_{1} \phi_{0}\left(z_{1}, z_{2}\right)=\omega\left(\tilde{z}_{2}\right), & \tilde{z}_{2}:=z_{2}\left(1-z_{1}\right) .
\end{aligned}
$$

Upon the action of $\Theta_{1}$, the second equation $\mathcal{D}_{2} \phi_{0}=0$ reduces to the Picard-Fuchs equation of the corresponding torus in terms of the variable $\tilde{z}_{2}$ :

$$
\begin{aligned}
\boldsymbol{E}_{0}: & \Theta_{1} \mathcal{D}_{2} \phi_{0}\left(z_{1}, z_{2}\right)=3 \mathcal{D}_{\text {ell }}^{(6)} \omega\left(\tilde{z}_{2}\right), \\
\boldsymbol{E}_{\tilde{1}}: & \Theta_{1} \mathcal{D}_{2} \phi_{0}\left(z_{1}, z_{2}\right)=4 \mathcal{D}_{\text {ell }}^{(5)} \omega\left(\tilde{z}_{2}\right), \\
\boldsymbol{E}_{5,6,7,8}: & \Theta_{1} \mathcal{D}_{2} \phi_{0}\left(z_{1}, z_{2}\right)=\frac{1}{1-z_{1}} \mathcal{D}_{\text {ell }}^{(N)} \omega\left(\tilde{z}_{2}\right),
\end{aligned}
$$

that is, $\omega\left(\tilde{z}_{2}\right)$ is a period of the elliptic curve.

We have thus arrived at the representation of the general solution $\phi_{0}$ for the PicardFuchs system in terms of a period $\omega$ of the fiber torus, which is closely related to the Seiberg-Witten periods:

$$
\begin{aligned}
\boldsymbol{E}_{0}: & \phi_{0}\left(z_{1}, z_{2}\right) & =\int^{z_{1}} \frac{\mathrm{d} z_{1}}{z_{1}} \frac{1}{1+z_{1}} \omega\left(\frac{z_{2}}{\left(1+z_{1}\right)^{3}}\right)+c\left(z_{2}\right), \\
\boldsymbol{E}_{\tilde{1}}: & \phi_{0}\left(z_{1}, z_{2}\right) & =\int^{z_{1}} \frac{\mathrm{d} z_{1}}{z_{1}} \frac{1}{1+z_{1}} \omega\left(\frac{z_{2}}{\left(1+z_{1}\right)^{2}}\right)+c\left(z_{2}\right), \\
\boldsymbol{E}_{5,6,7,8}: & \phi_{0}\left(z_{1}, z_{2}\right) & =\int^{z_{1}} \frac{\mathrm{d} z_{1}}{z_{1}} \omega\left(z_{2}-z_{1} z_{2}\right)+c\left(z_{2}\right),
\end{aligned}
$$


where $c\left(z_{2}\right)$ is a counterterm to ensure $\mathcal{D}_{2} \phi_{0}=0$.

Curiously, the deformation of the single-variable function $\omega\left(z_{2}\right)$ to the one appeared in the right hand side of (4.53), (4.54) and (4.55) admits the following description in terms of the differential operator $\mathcal{L}_{n}^{(N)}$ respectively:

$$
\begin{aligned}
\frac{1}{1+z_{1}} \omega\left(\frac{z_{2}}{\left(1+z_{1}\right)^{3}}\right) & =\left(1+\sum_{n=1}^{\infty} n z_{1}^{n} \mathcal{L}_{n}^{(0)}\right) \omega\left(z_{2}\right), \\
\frac{1}{1+z_{1}} \omega\left(\frac{z_{2}}{\left(1+z_{1}\right)^{2}}\right) & =\left(1+\sum_{n=1}^{\infty} n z_{1}^{n} \mathcal{L}_{n}^{(\tilde{1})}\right) \omega\left(z_{2}\right), \\
\omega\left(z_{2}-z_{1} z_{2}\right) & =\left(1+\sum_{n=1}^{\infty} n z_{1}^{n} \mathcal{L}_{n}^{(N)}\right) \omega\left(z_{2}\right), \quad N=5,6,7,8 .
\end{aligned}
$$

For the period $\phi$, we obtain the following integral formula: for $\boldsymbol{E}_{0}$ and $\boldsymbol{E}_{\tilde{1}}$ models,

$$
\begin{aligned}
& \boldsymbol{E}_{0}: \phi^{(0)}\left(z_{1}, z_{2}\right)=\int_{\epsilon}^{z_{1}} \frac{\mathrm{d} z_{1}}{z_{1}} \frac{1}{1+z_{1}} \varpi^{(6)}\left(\frac{z_{2}}{\left(1+z_{1}\right)^{3}}\right)+\xi^{(0)}\left(z_{2}\right), \\
& \boldsymbol{E}_{\tilde{1}}: \phi^{(\tilde{1})}\left(z_{1}, z_{2}\right)=\int_{\epsilon}^{z_{1}} \frac{\mathrm{d} z_{1}}{z_{1}} \frac{1}{1+z_{1}} \varpi^{(5)}\left(\frac{z_{2}}{\left(1+z_{1}\right)^{2}}\right)+\xi^{(\tilde{1})}\left(z_{2}\right),
\end{aligned}
$$

and for the principal series

$$
\begin{aligned}
\boldsymbol{E}_{5,6,7,8}: \phi^{(N)}\left(z_{1}, z_{2}\right) & =\int_{\epsilon}^{z_{1}} \frac{\mathrm{d} z_{1}}{z_{1}} \varpi^{(N)}\left(z_{2}-z_{1} z_{2}\right)+\xi^{(N)}\left(z_{2}\right) \\
& =\int_{1}^{z_{1}} \frac{\mathrm{d} z_{1}}{z_{1}} \varpi^{(N)}\left(z_{2}-z_{1} z_{2}\right),
\end{aligned}
$$

where we must discard a $\log (\epsilon)$ term before taking the limit $\epsilon \rightarrow 0$. The last equation implies that for the principal series, $\phi$ is a vanishing period at $z_{1}=1$.

Let $\tilde{\tau}$ be the coupling constant of the Seiberg-Witten theory with the bare parameters $\left(z_{1}, z_{2}\right)$. It is given by the deformed mirror map

$$
2 \pi \mathbf{i} \tilde{\tau}=\frac{\varpi_{D}\left(\tilde{z}_{2}\right)}{\varpi\left(\tilde{z}_{2}\right)},
$$

where $\tilde{z}_{2}=\tilde{z}_{2}\left(z_{1}, z_{2}\right)$ is given in (4.53), (4.54), and (4.55) respectively.

We can also show using (4.62), (4.63), (4.64), that the instanton part of the period $\phi_{D}$ can be written as a Seiberg-Witten period, where we have no need to introduce the 
cut-off parameter $\epsilon$ in contrast to the case of $\phi$ :

$$
\begin{aligned}
& \boldsymbol{E}_{0}:-\frac{1}{2 \pi \mathrm{i}} \sum_{n=1}^{\infty}\left(\frac{f_{n}^{(0)}}{\varpi^{(6)}}\right)\left(z_{2}\right) z_{1}^{n}=\int_{0}^{z_{1}} \frac{\mathrm{d} z_{1}}{z_{1}} \frac{1}{1+z_{1}}(\tilde{\tau}-\tau) \varpi^{(6)}\left(\frac{z_{2}}{\left(1+z_{1}\right)^{3}}\right), \\
& \boldsymbol{E}_{\tilde{1}}:-\frac{1}{2 \pi \mathrm{i}} \sum_{n=1}^{\infty}\left(\frac{f_{n}^{(\tilde{1})}}{\varpi^{(5)}}\right)\left(z_{2}\right) z_{1}^{n}=\int_{0}^{z_{1}} \frac{\mathrm{d} z_{1}}{z_{1}} \frac{1}{1+z_{1}}(\tilde{\tau}-\tau) \varpi^{(5)}\left(\frac{z_{2}}{\left(1+z_{1}\right)^{2}}\right), \\
& \boldsymbol{E}_{5,6,7,8}:-\frac{1}{2 \pi \mathrm{i}} \sum_{n=1}^{\infty}\left(\frac{f_{n}^{(N)}}{\varpi^{(N)}}\right)\left(z_{2}\right) z_{1}^{n}=\int_{0}^{z_{1}} \frac{\mathrm{d} z_{1}}{z_{1}}(\tilde{\tau}-\tau) \varpi^{(N)}\left(z_{2}-z_{1} z_{2}\right) .
\end{aligned}
$$

Note that in particular (4.71) for the $\boldsymbol{E}_{8}$ model corresponds to the formula [29, (3.5)] obtained by direct evaluation of the Seiberg-Witten periods from the curve (9.6). The integration variable $v$ there satisfies $E_{4}(\tilde{\tau})^{3} / E_{6}(\tilde{\tau})^{2}=E_{4}(\tau)^{3} /\left(E_{6}(\tau)-v\right)^{2}$, from which we see the correspondence of the bare variables

$$
v:=\left(\frac{27}{\pi^{6}}\right) \frac{1}{u}=-2 E_{4}(\tau)^{3 / 2} z_{2} z_{1}=\left[E_{6}(\tau)-E_{4}(\tau)^{3 / 2}\right] z_{1}
$$

\section{Genus Zero Partition Functions}

\subsection{Recursion relations}

In order to obtain the instanton expansion of the genus zero potential $F_{0}$, we have to convert the two sequences of functions of $z_{2}$ :

$$
c_{n}=\frac{\mathcal{L}_{n} \varpi}{\varpi}, \quad f_{n}=-\left(\varpi \mathcal{L}_{n} \varpi_{D}-\varpi_{D} \mathcal{L}_{n} \varpi\right)
$$

into modular functions of $\Gamma_{\text {ell }}$, which is achieved by finding the recursion relations for $\left\{c_{n}\right\}$ and $\left\{f_{n}\right\}$. To this end, let us make the ansatz $c_{n}(\tau)=B_{n} e_{2}(\tau)+D_{n}$, with $e_{2 k}:=E_{2 k} \varpi^{-2 k}$. It will become clear from the recursion relations that $B_{n}, D_{n}, f_{n}$ are degree $n$ polynomials in $y$, where $y=\left(1-z_{2}\right)^{-1}$; in particular they are all anomaly-free modular functions of $\Gamma_{\text {ell }}$.

The equations (3.19) enable us to evaluate the following logarithmic derivatives that are indispensable to establish the recursion relations:

$$
\begin{aligned}
\Theta_{2} \varpi & =\frac{\varpi}{12}\left\{y e_{2}-[(10-N)-(9-N) y]\right\}, \\
\Theta_{2}\left(e_{2} \varpi\right) & =\frac{\varpi}{12}\left\{-y e_{4}+e_{2}[(10-N)-(9-N) y]\right\},
\end{aligned}
$$

where $\varpi$ is the fundamental period of the $\boldsymbol{E}_{N}$ elliptic curves with $N=5,6,7,8$. 
Principal series For them, from the relation $(n+1)^{2} \mathcal{L}_{n+1}=-n\left(\Theta_{2}-n\right) \mathcal{L}_{n}$,

$$
\varpi c_{n+1}=-\frac{n}{(n+1)^{2}}\left(\Theta_{2}-n\right)\left(\varpi c_{n}\right) .
$$

Then the equations (5.1) and (5.2) yields the recursion relation for $\left\{c_{n}\right\}$ :

$$
\left(\begin{array}{c}
B_{n+1} \\
D_{n+1}
\end{array}\right)=-\frac{n}{(n+1)^{2}}\left[y(y-1) \frac{\mathrm{d}}{\mathrm{d} y}+\left(\begin{array}{cc}
D_{1}-n & -B_{1} \\
e_{4} B_{1} & -D_{1}-n
\end{array}\right)\right]\left(\begin{array}{c}
B_{n} \\
D_{n}
\end{array}\right),
$$

where $e_{4}=\left(16-16 y^{-1}+y^{-2}\right),\left(9-8 y^{-1}\right),\left(4-3 y^{-1}\right), 1$, for $N=5,6,7,8$ respectively. Note that we have replaced $\Theta_{2}$ by $y(y-1) \mathrm{d} / \mathrm{d} y$ in the recursion relation above because $B_{n}$ and $D_{n}$ depend on $z_{2}$ only through $y$.

Since $c_{1}=-\Theta_{2} \varpi / \varpi$, the first term $\left(B_{1}, D_{1}\right)$ can be seen immediately from (5.1):

$$
B_{1}=-\frac{y}{12}, \quad D_{1}=\frac{1}{12}[(10-N)-(9-N) y] .
$$

The recursion relation for $\left\{f_{n}\right\}$ can be obtained in a similar manner:

$$
f_{n+1}=-\frac{n}{(n+1)^{2}}\left\{\left[y(y-1) \frac{\mathrm{d}}{\mathrm{d} y}-n+c_{1}\right] f_{n}-f_{1} c_{n}\right\}, \quad f_{1}=y .
$$

Furthermore $\left\{f_{n}\right\}$ and $\left\{c_{n}=B_{n} e_{2}+D_{n}\right\}$ are related each other by

$$
B_{n}=-\frac{1}{12} f_{n}, \quad D_{n}=\frac{1}{y}\left\{\frac{(n+1)^{2}}{n} f_{n+1}+\left[y(y-1) \frac{\mathrm{d}}{\mathrm{d} y}-n+D_{1}\right] f_{n}\right\} .
$$

Here we list the first few elements of $\left\{\left(B_{n}, D_{n}\right)\right\}$ only for the $\boldsymbol{E}_{8}$ model:

$$
\begin{aligned}
B_{1} & =-\frac{1}{12} y, D_{1}=\frac{1}{12}(2-y), B_{2}=\frac{1}{48} y(y-2), D_{2}=\frac{1}{144}\left(7-7 y+3 y^{2}\right), \\
B_{3} & =-\frac{1}{7776} y\left(211-211 y+72 y^{2}\right), D_{3}=-\frac{1}{7776}(y-2)\left(72 y^{2}-91 y+91\right), \\
B_{4} & =\frac{1}{10368} y(y-2)\left(54 y^{2}-103 y+103\right), \\
D_{4} & =\frac{1}{124416}\left(1729-3458 y+4477 y^{2}-2748 y^{3}+648 y^{4}\right) .
\end{aligned}
$$

$\boldsymbol{E}_{\tilde{1}}$ model The procedure to get the recursion relations for $\left\{c_{n}\right\}$ and $\left\{f_{n}\right\}$ are similar to the case of principal series. In this case the $\boldsymbol{E}_{5}$ torus is relevant. 
Using $(n+1)^{2} \mathcal{L}_{n+1}=-n\left(2 \Theta_{2}+n+1\right) \mathcal{L}_{n}$ we get the recursion relation for $\left\{c_{n}\right\}$

$$
\left(\begin{array}{c}
B_{n+1} \\
D_{n+1}
\end{array}\right)=-\frac{n}{(n+1)^{2}}\left[2 y(y-1) \frac{\mathrm{d}}{\mathrm{d} y}+\left(\begin{array}{cc}
D_{1}+n+2 & -B_{1} \\
e_{4} B_{1} & -D_{1}+n
\end{array}\right)\right]\left(\begin{array}{c}
B_{n} \\
D_{n}
\end{array}\right),
$$

with $\left(B_{1}, D_{1}\right)=(-y / 6,-(4 y+1) / 6)$ and $e_{4}=\left(16-16 y^{-1}+y^{-2}\right)$.

The recursion relation for $\left\{f_{n}\right\}$ reads

$$
f_{n+1}=-\frac{n}{(n+1)^{2}}\left\{\left[2 y(y-1) \frac{\mathrm{d}}{\mathrm{d} y}+2+n+c_{1}\right] f_{n}-f_{1} c_{n}\right\}, \quad f_{1}=2 y .
$$

The relation between $\left\{f_{n}\right\}$ and $\left\{c_{n}\right\}$ is

$$
B_{n}=-\frac{1}{12} f_{n}, \quad D_{n}=\frac{1}{2 y}\left\{\frac{(n+1)^{2}}{n} f_{n+1}+\left[2 y(y-1) \frac{\mathrm{d}}{\mathrm{d} y}+2+n+D_{1}\right] f_{n}\right\} .
$$

We list the first few members:

$$
\begin{aligned}
& B_{1}=-\frac{1}{6} y, D_{1}=-\frac{1}{6}(1+4 y), B_{2}=\frac{1}{24} y(2 y+1), D_{2}=\frac{1}{24}\left(8 y^{2}+1\right), \\
& B_{3}=-\frac{1}{108} y\left(8 y^{2}+y+2\right), D_{3}=-\frac{1}{108}(1+4 y)\left(8 y^{2}-5 y+2\right), \\
& B_{4}=\frac{1}{288} y\left(24 y^{3}-4 y^{2}+2 y+3\right), D_{4}=\frac{1}{288}\left(96 y^{4}-64 y^{3}+7 y^{2}+5 y+3\right) .
\end{aligned}
$$

$\boldsymbol{E}_{\mathbf{0}}$ model This case has been analyzed in [18], which we briefly repeat here for convenience. Recall that underlying torus model is $\boldsymbol{E}_{6}$.

First, the relation among the operators $(n+1)^{2} \mathcal{L}_{n+1}=-n\left(3 \Theta_{2}+n+1\right) \mathcal{L}_{n}$ yields

$$
\left(\begin{array}{c}
B_{n+1} \\
D_{n+1}
\end{array}\right)=-\frac{n}{(n+1)^{2}}\left[3 y(y-1) \frac{\mathrm{d}}{\mathrm{d} y}+\left(\begin{array}{cc}
D_{1}+n+2 & -B_{1} \\
e_{4} B_{1} & -D_{1}+n
\end{array}\right)\right]\left(\begin{array}{c}
B_{n} \\
D_{n}
\end{array}\right),
$$

with $\left(B_{1}, D_{1}\right)=(-y / 4,-3 y / 4)$ and $e_{4}=\left(9-8 y^{-1}\right)$.

The recursion relation for $\left\{f_{n}\right\}$ becomes

$$
f_{n+1}=-\frac{n}{(n+1)^{2}}\left\{\left[3 y(y-1) \frac{\mathrm{d}}{\mathrm{d} y}+n+2+c_{1}\right] f_{n}-c_{n} f_{1}\right\}, \quad f_{1}=3 y,
$$

with the relation between $\left\{f_{n}\right\}$ and $\left\{c_{n}\right\}$

$$
B_{n}=-\frac{1}{12} f_{n}, D_{n}=\frac{1}{3 y}\left\{\frac{(n+1)^{2}}{n} f_{n+1}+\left[3 y(y-1) \frac{\mathrm{d}}{\mathrm{d} y}+n+2+D_{1}\right] f_{n}\right\} .
$$


The first few members of $\left\{\left(B_{n}, D_{n}\right)\right\}$ are

$$
\begin{aligned}
& B_{1}=-\frac{1}{4} y, D_{1}=-\frac{3}{4} y, B_{2}=\frac{3}{16} y^{2}, D_{2}=\frac{1}{16} y(9 y-4), \\
& B_{3}=-\frac{1}{72} y^{2}(18 y-7), D_{3}=-\frac{1}{72} y\left(54 y^{2}-45 y+4\right), \\
& B_{4}=\frac{1}{192} y^{2}(27 y-2)(3 y-2), D_{4}=\frac{1}{192} y^{2}\left(243 y^{2}-288 y+68\right) .
\end{aligned}
$$

Finally, we have observed for any of the six models that

$$
\frac{f_{n}(y)}{\varpi\left(z_{2}\right)^{2}}=-12 \frac{\partial c_{n}(\tau)}{\partial E_{2}(\tau)},
$$

which turns out to be of fundamental importance both for the instanton expansion and for the investigation of the modular anomaly equations of the genus zero and one GromovWitten potentials below.

\subsection{Instanton expansion}

The instanton expansion of the genus zero potential $F_{0}$ is obtained by conversion of $z_{1}$ and $z_{2}$ in $F_{0}$ to the function of $q:=\mathrm{e}^{2 \pi \mathrm{i} \tau}$ and $p:=\mathrm{e}^{2 \pi \mathrm{i} \sigma}$. We define the genus zero Gromov-Witten invariant $N_{0 ; n, m} \in \mathbf{Q}$ of bidegree $(n, m)$ by the $q$-expansion of $Z_{0 ; n}(\tau)$

$$
Z_{0 ; n}(\tau)=\sum_{m=0}^{\infty} N_{0 ; n, m} q^{m}
$$

We find a useful expression of the genus zero potential:

$$
\frac{1}{2 \pi \mathrm{i}}\left(\frac{\partial F_{0}}{\partial \sigma}\right)=\Theta_{p} F_{0}=12 h\left(\frac{\partial z_{1}}{\partial E_{2}}\right)\left(\frac{1}{\tilde{p}} \frac{\partial \tilde{p}}{\partial z_{1}}\right),
$$

which follows from the fact that $\tilde{p}$ does not depend on $E_{2}$ :

$$
\left(\frac{d \tilde{p}}{d E_{2}}\right)=\left(\frac{\partial z_{1}}{\partial E_{2}}\right)\left(\frac{\partial \tilde{p}}{\partial z_{1}}\right)+\sum_{n=1}^{\infty}\left(\frac{\partial c_{n}}{\partial E_{2}}\right)\left(\frac{\partial \tilde{p}}{\partial c_{n}}\right)=0,
$$

because $\tilde{p}=p \psi(\tau)$ with $\psi(\tau)$ anomaly-free, and the substitution of the identity (5.3).

Then we see from the expansion of the right hand side of (5.5) that the general form of the partition function $Z_{0 ; n}(\tau)$ reads

$$
\begin{aligned}
& Z_{0 ; 1}=(9-N)\left(\frac{y \psi}{\varpi^{2}}\right), \quad N=0, \tilde{1}, 5,6,7,8, \\
& Z_{0 ; n}=\left(Z_{0 ; 1}\right)^{n} \varpi^{2(n-1)} \mathcal{P}_{0 ; n-1}\left(e_{2}, y^{-1}\right),
\end{aligned}
$$


where $\mathcal{P}_{0 ; n-1}$ is a degree $n-1$ polynomial in $e_{2}=E_{2}(\tau) / \varpi\left(z_{2}\right)^{2}$ and $y^{-1}=1-z_{2}$. The relation between $Z_{0 ; 1}$ and the special value of the $\boldsymbol{E}_{8}$ theta function prescribed by the Kähler class $J^{(N)}$ defined in (4.17)-(4.21) will be discussed later.

As space is limited, we give only the first four terms of the $p$-expansions of (5.5):

$$
\begin{aligned}
& \Theta_{p} F_{0}^{(0)}=9 \frac{y \tilde{p}}{\varpi^{2}}+\frac{(y \tilde{p})^{2}}{\varpi^{2}} \frac{9}{4} e_{2}+\frac{(y \tilde{p})^{3}}{\varpi^{2}} \frac{1}{32}\left(27 e_{2}^{2}+45-\frac{40}{y}\right) \\
& +\frac{(y \tilde{p})^{4}}{\varpi^{2}} \frac{1}{32}\left[12 e_{2}^{3}+e_{2}\left(45-\frac{40}{y}\right)-27+\frac{36}{y}-\frac{8}{y^{2}}\right] \\
& \Theta_{p} F_{0}^{(\tilde{1})}=8 \frac{y \tilde{p}}{\varpi^{2}}+\frac{(y \tilde{p})^{2}}{\varpi^{2}} \frac{2}{3}\left(2 e_{2}+2-\frac{1}{y}\right)+\frac{(y \tilde{p})^{3}}{\varpi^{2}} \frac{1}{9}\left[3 e_{2}^{2}+e_{2}\left(6-\frac{3}{y}\right)+8-\frac{8}{y}+\frac{2}{y^{2}}\right] \\
& +\frac{(y \tilde{p})^{4}}{\varpi^{2}} \frac{1}{162}\left[16 e_{2}^{3}+e_{2}^{2}\left(48-\frac{24}{y}\right)+e_{2}\left(108-\frac{108}{y}+\frac{27}{y^{2}}\right)+40-\frac{60}{y}+\frac{48}{y^{2}}-\frac{14}{y^{3}}\right], \\
& \Theta_{p} F_{0}^{(5)}=4 \frac{y \tilde{p}}{\varpi^{2}}+\frac{(y \tilde{p})^{2}}{\varpi^{2}} \frac{1}{3}\left(e_{2}+1+\frac{1}{y}\right)+\frac{(y \tilde{p})^{3}}{\varpi^{2}} \frac{1}{72}\left[3 e_{2}^{2}+e_{2}\left(6+\frac{6}{y}\right)+8+\frac{4}{y}+\frac{5}{y^{2}}\right] \\
& +\frac{(y \tilde{p})^{4}}{\varpi^{2}} \frac{1}{648}\left[4 e_{2}^{3}+e_{2}^{2}\left(12+\frac{12}{y}\right)+e_{2}\left(27+\frac{18}{y}+\frac{18}{y^{2}}\right)+10+\frac{39}{y}+\frac{12}{y^{2}}+\frac{10}{y^{3}}\right], \\
& \Theta_{p} F_{0}^{(6)}=3 \frac{y \tilde{p}}{\varpi^{2}}+\frac{(y \tilde{p})^{2}}{\varpi^{2}} \frac{1}{4}\left(e_{2}+\frac{2}{y}\right)+\frac{(y \tilde{p})^{3}}{\varpi^{2}} \frac{1}{864}\left(27 e_{2}^{2}+108 \frac{e_{2}}{y}+45-\frac{4}{y}+\frac{112}{y^{2}}\right) \\
& +\frac{(y \tilde{p})^{4}}{\varpi^{2}} \frac{1}{2592}\left[12 e_{2}^{3}+72 \frac{e_{2}^{2}}{y}+e_{2}\left(45-\frac{4}{y}+\frac{148}{y^{2}}\right)-27+\frac{144}{y}-\frac{8}{y^{2}}+\frac{104}{y^{3}}\right], \\
& \Theta_{p} F_{0}^{(7)}=2 \frac{y \tilde{p}}{\varpi^{2}}+\frac{(y \tilde{p})^{2}}{\varpi^{2}} \frac{1}{6}\left(e_{2}-1+\frac{3}{y}\right)+\frac{(y \tilde{p})^{3}}{\varpi^{2}} \frac{1}{288}\left[6 e_{2}^{2}-e_{2}\left(12-\frac{36}{y}\right)+16-\frac{33}{y}+\frac{51}{y^{2}}\right] \\
& +\frac{(y \tilde{p})^{4}}{\varpi^{2}} \frac{1}{2592}\left[8 e_{2}^{3}-e_{2}^{2}\left(24-\frac{72}{y}\right)+e_{2}\left(54-\frac{135}{y}+\frac{207}{y^{2}}\right)-56+\frac{189}{y}-\frac{180}{y^{2}}+\frac{189}{y^{3}}\right], \\
& \Theta_{p} F_{0}^{(8)}=\frac{y \tilde{p}}{\varpi^{2}}+\frac{(y \tilde{p})^{2}}{\varpi^{2}} \frac{1}{12}\left(e_{2}-2+\frac{4}{y}\right)+\frac{(y \tilde{p})^{3}}{\varpi^{2}} \frac{1}{2592}\left[27 e_{2}^{2}-e_{2}\left(108-\frac{216}{y}\right)+153-\frac{394}{y}\right. \\
& \left.+\frac{394}{y^{2}}\right]+\frac{(y \tilde{p})^{4}}{\varpi^{2}} \frac{1}{7776}\left[12 e_{2}^{3}-e_{2}^{2}\left(72-\frac{144}{y}\right)+e_{2}\left(189-\frac{538}{y}+\frac{538}{y^{2}}\right)-213+\frac{734}{y}\right. \\
& \left.-\frac{924}{y^{2}}+\frac{616}{y^{3}}\right]
\end{aligned}
$$

At this point it would be helpful to mention in advance the general form of higher genus Gromov-Witten partition functions $Z_{g ; n}(\tau)$, which is indeed predicted from the modular anomaly equation (2.17),

$$
Z_{g ; n}=\left(Z_{0 ; 1}\right)^{n} \varpi^{2(g+n-1)} \mathcal{P}_{g ; g+n-1}\left(e_{2}, y^{-1}\right)
$$


where $\mathcal{P}_{g ; g+n-1}$ is a degree $g+n-1$ polynomial in $e_{2}$ and $y^{-1}$.

\subsection{Partition functions and modular forms}

It is of primary importance to ensure the relation between the singly wound partition function $Z_{0 ; 1}(\tau)$ and the $\boldsymbol{E}_{8}$ theta function (2.14) as a consistency check of our formalism.

The specializations of the $\boldsymbol{E}_{8}$ theta function to the Kähler moduli of the $\boldsymbol{E}_{\tilde{1}}$ and $\boldsymbol{E}_{5}$ models, for example, are calculated as follows:

$$
\begin{aligned}
\tilde{\boldsymbol{E}}_{1}: \Theta_{\boldsymbol{E}_{8}}\left(4 \tau \mid \omega_{2} \tau\right) & =\frac{1}{2} q^{-\frac{3}{2}}\left(\sum_{a=2,3} \vartheta_{a}\left(4 \tau \mid \frac{\tau}{2}\right)^{8}-\sum_{b=1,4} \vartheta_{b}\left(4 \tau \mid \frac{\tau}{2}\right)^{8}\right) \\
& =\frac{1}{2} q^{-1}\left(\sum_{a=2,3} \vartheta_{a}(4 \tau)^{2} \vartheta_{a}(4 \tau \mid \tau)^{6}-\vartheta_{4}(4 \tau)^{2} \vartheta_{4}(4 \tau \mid \tau)^{6}\right), \\
\boldsymbol{E}_{5}: \Theta_{\boldsymbol{E}_{8}}\left(4 \tau \mid \omega_{5} \tau\right) & =\frac{1}{2} q^{-1}\left(\sum_{a=2,3} \vartheta_{a}(4 \tau)^{4} \vartheta_{a}(4 \tau \mid \tau)^{4}-\vartheta_{4}(4 \tau)^{4} \vartheta_{4}(4 \tau \mid \tau)^{4}\right) .
\end{aligned}
$$

We find that $Z_{0 ; 1}$ can indeed be written by the $E_{8}$ theta function with the prescribed Kähler class $J^{(N)}$ as predicted in (2.14), in addition to the fact that it admits a concise description by the Dedekind eta function:

$$
\begin{aligned}
& Z_{0 ; 1}^{(0)}(\tau)=9\left(\frac{y \psi}{\varpi^{2}}\right)^{(0)}\left(z_{2}\right)=9 q^{\frac{1}{6}} \frac{1}{\eta(\tau)^{4}}=Z_{0 ; 1}\left(3 \tau \mid \omega_{8} \tau\right), \\
& Z_{0 ; 1}^{(\tilde{1})}(\tau)=8\left(\frac{y \psi}{\varpi^{2}}\right)^{(\tilde{1})}\left(z_{2}\right)=8 q^{\frac{1}{4}} \frac{1}{\eta(\tau)^{2} \eta(2 \tau)^{2}}=Z_{0 ; 1}\left(4 \tau \mid \omega_{2} \tau\right), \\
& Z_{0 ; 1}^{(5)}(\tau)=4\left(\frac{y \psi}{\varpi^{2}}\right)^{(5)}\left(z_{2}\right)=4 q^{\frac{1}{2}} \frac{\varpi^{(5)}\left(z_{2}\right)}{\eta(2 \tau)^{6}}=Z_{0 ; 1}\left(4 \tau \mid \omega_{5} \tau\right), \\
& Z_{0 ; 1}^{(6)}(\tau)=3\left(\frac{y \psi}{\varpi^{2}}\right)^{(6)}\left(z_{2}\right)=3 q^{\frac{1}{2}} \frac{\varpi^{(6)}\left(z_{2}\right)}{\eta(\tau)^{3} \eta(3 \tau)^{3}}=Z_{0 ; 1}\left(3 \tau \mid \omega_{6} \tau\right), \\
& Z_{0 ; 1}^{(7)}(\tau)=2\left(\frac{y \psi}{\varpi^{2}}\right)^{(7)}\left(z_{2}\right)=2 q^{\frac{1}{2}} \frac{\varpi^{(7)}\left(z_{2}\right)^{2}}{\eta(\tau)^{4} \eta(2 \tau)^{4}}=Z_{0 ; 1}\left(2 \tau \mid \omega_{7} \tau\right), \\
& Z_{0 ; 1}^{(8)}(\tau)=\left(\frac{y \psi}{\varpi_{0}^{2}}\right)^{(8)}\left(z_{2}\right)=q^{\frac{1}{2}} \frac{\varpi^{(8)}\left(z_{2}\right)^{4}}{\eta(\tau)^{12}}=Z_{0 ; 1}(\tau \mid 0) .
\end{aligned}
$$

$Z_{0 ; 1}(\tau)$ of the $\boldsymbol{E}_{3}$ and the $\boldsymbol{E}_{4}$ model calculated form the $\boldsymbol{E}_{8}$ theta function using (2.14) 
can also be expressed by the eta functions:

$$
\begin{aligned}
& \boldsymbol{E}_{3}: \frac{\Theta_{\boldsymbol{E}_{8}}\left(6 \tau \mid \omega_{3} \tau\right)}{\varphi(6 \tau)^{12}}=\frac{6 q^{\frac{1}{2}}}{\eta(\tau) \eta(2 \tau) \eta(3 \tau) \eta(6 \tau)}, \\
& \boldsymbol{E}_{4}: \frac{\Theta_{\boldsymbol{E}_{8}}\left(5 \tau \mid \omega_{4} \tau\right)}{\varphi(5 \tau)^{12}}=\frac{5 q^{\frac{1}{2}}}{\eta(\tau)^{2} \eta(5 \tau)^{2}} .
\end{aligned}
$$

As for partition functions of multiple winding number, we here give the expressions of those in terms of modular forms only for the $\boldsymbol{E}_{7}$ and $\boldsymbol{E}_{8}$ models; the latter has originally been obtained in [29].

$\boldsymbol{E}_{7}$ model $\quad$ Let $\chi:=Z_{0 ; 1}^{(7)} / A=2 q^{1 / 2} /(\eta(\tau) \eta(2 \tau))^{4}$, see (7.1).

$$
\begin{aligned}
Z_{0 ; 1}^{(7)} & =\chi A, \\
Z_{0 ; 2}^{(7)} & =\frac{\chi^{2}}{48} A\left(A E_{2}-A^{2}+3 B\right), \\
Z_{0 ; 3}^{(7)} & =\frac{\chi^{3}}{6912} A\left(6 E_{2}^{2} A^{2}-12 E_{2} A^{3}+36 E_{2} A B+16 A^{4}-33 A^{2} B+51 B^{2}\right), \\
Z_{0 ; 4}^{(7)} & =\frac{\chi^{4}}{165888} A\left(-56 A^{6}+189 A^{4} B-180 B^{2} A^{2}+189 B^{3}+8 E_{2}^{3} A^{3}-24 E_{2}^{2} A^{4}+72 E_{2}^{2} A^{2} B\right. \\
& \left.+54 E_{2} A^{5}-135 E_{2} A^{3} B+207 E_{2} A B^{2}\right), \\
Z_{0 ; 5}^{(7)} & =\frac{\chi^{5}}{1990656000} A\left(123328 A^{8}-514272 A^{6} B+858987 A^{4} B^{2}+6250 A^{4} E_{2}^{4}-25000 A^{5} E_{2}^{3}\right. \\
& +406215 B^{4}-585000 A^{3} E_{2} B^{2}+499500 A^{5} E_{2} B+607500 A E_{2} B^{3}-213750 A^{4} E_{2}^{2} B \\
& \left.+326250 A^{2} E_{2}^{2} B^{2}-508680 A^{2} B^{3}-136000 A^{7} E_{2}+75000 A^{3} E_{2}^{3} B+75000 A^{6} E_{2}^{2}\right) .
\end{aligned}
$$

\section{$\boldsymbol{E}_{8}$ model}

$$
\begin{aligned}
Z_{0 ; 1}^{(8)} & =\frac{1}{\varphi^{12}} E_{4} \\
Z_{0 ; 2}^{(8)} & =\frac{1}{24 \varphi^{24}} E_{4}\left(2 E_{6}+E_{2} E_{4}\right) \\
Z_{0 ; 3}^{(8)} & =\frac{1}{15552 \varphi^{36}} E_{4}\left(109 E_{4}^{3}+197 E_{6}^{2}+216 E_{2} E_{4} E_{6}+54 E_{2}^{2} E_{4}^{2}\right), \\
Z_{0 ; 4}^{(8)} & =\frac{1}{62208 \varphi^{48}} E_{4}\left(272 E_{4}^{3} E_{6}+154 E_{6}^{3}+109 E_{2} E_{4}^{4}+269 E_{2} E_{4} E_{6}^{2}+144 E_{2}^{2} E_{4}^{2} E_{6}\right.
\end{aligned}
$$




$$
\begin{aligned}
& \left.+24 E_{2}^{3} E_{4}^{3}\right), \\
Z_{0 ; 5}^{(8)} & =\frac{1}{37324800 \varphi^{60}} E_{4}\left(426250 E_{2}^{2} E_{4}^{2} E_{6}^{2}+150000 E_{2}^{3} E_{4}^{3} E_{6}+207505 E_{6}^{4}+136250 E_{2}^{2} E_{4}^{5}\right. \\
& \left.+772460 E_{4}^{3} E_{6}^{2}+116769 E_{4}^{6}+18750 E_{2}^{4} E_{4}^{4}+653000 E_{2} E_{4}^{4} E_{6}+505000 E_{2} E_{4} E_{6}^{3}\right) .
\end{aligned}
$$

\subsection{Modular anomaly equation}

We are now ready to give the modular anomaly equation, which determines the $E_{2}$ dependence of the genus zero partition function $Z_{0 ; n}(\tau)$ closely following $[29,18]$.

First by differentiation of (4.52) with respect to $E_{2}(\tau)$, we obtain

$$
\frac{\partial}{\partial E_{2}}\left(\Theta_{p} F_{0}\right)=h \sum_{n=1}^{\infty} \frac{f_{n}}{\varpi^{2}} n z_{1}^{n-1}\left(\frac{\partial z_{1}}{\partial E_{2}}\right) .
$$

Then the substitution of (5.5) to the above equation brings about

$$
\Theta_{p}\left[24 h\left(\frac{\partial F_{0}}{\partial E_{2}}\right)-\left(\Theta_{p} F_{0}\right)^{2}\right]=0 .
$$

Eventually we arrive at the modular anomaly equation for genus zero:

$$
h\left(\frac{\partial F_{0}}{\partial E_{2}}\right)=\frac{1}{24}\left(\Theta_{p} F_{0}\right)^{2} .
$$

Using the definition of the potential $F_{0}=\sum_{n=1}^{\infty} Z_{0 ; n}(\tau) p^{n}$, the anomaly equation can be rewritten as

$$
h \frac{\partial Z_{0 ; n}(\tau)}{\partial E_{2}(\tau)}=\frac{1}{24} \sum_{k=1}^{n-1} k(n-k) Z_{0 ; k}(\tau) Z_{0 ; n-k}(\tau),
$$

which is consistent with the general form of the anomaly equation (2.17). The appearance of the normalization factor $h$ (4.52) in the left hand side is explained by the fact that $h E_{2}(h \tau)-E_{2}(\tau)$ is an anomaly-free modular form of $\Gamma_{0}(h)$.

\subsection{Rational instanton numbers}

We denote the genus zero instanton number of bidegree $(n, m)$ by $N_{0 ; n, m}^{\text {inst }}$, which counts the 'number' of the rational curves of a given degree in the almost del Pezzo surface $B_{9}$, 
and we define its generating function with fixed $n$ by

$$
Z_{0 ; n}^{\text {inst }}(\tau)=\sum_{m=0}^{\infty} N_{0 ; n, m}^{\text {inst }} q^{m} .
$$

It is well-known that the genus zero multiple covering formula found in [5] leads to the following decomposition of the genus zero Gromov-Witten partition function:

$$
Z_{0 ; n}(\tau)=\sum_{k \mid n} k^{-3} Z_{0 ; \frac{n}{k}}^{\text {inst }}(k \tau)
$$

We can invert this equation using the Möbius function $\mu: \mathbf{N} \rightarrow\{0, \pm 1\}$ as

$$
Z_{0 ; n}^{\text {inst }}(\tau)=\sum_{k \mid n} \mu(k) k^{-3} Z_{0 ; \frac{n}{k}}(k \tau)
$$

Recall that the Möbius function $\mu$ is defined as follows: $\mu(1)=1, \mu(n)=(-1)^{l}$ if $n$ is factorized into $l$ distinct primes, and $\mu(n)=0$ if $n$ is not square-free.

We will give the first few terms of the expansions of $Z_{0 ; n}^{\text {inst }}$, for each models below.

\section{$E_{5}$ model}

$$
\begin{aligned}
Z_{0 ; 1}^{\text {inst }} & =4+16 q+40 q^{2}+96 q^{3}+220 q^{4}+464 q^{5}+920 q^{6}+1760 q^{7}+3276 q^{8}+5920 q^{9} \\
& +10408 q^{10}+\cdots \\
Z_{0 ; 2}^{\text {inst }} & =-20 q^{2}-128 q^{3}-608 q^{4}-2304 q^{5}-7672 q^{6}-23040 q^{7}-64256 q^{8}-168448 q^{9} \\
& -419908 q^{10}-\cdots, \\
Z_{0 ; 3}^{\text {inst }} & =48 q^{3}+588 q^{4}+4224 q^{5}+23112 q^{6}+105888 q^{7}+426624 q^{8}+1557216 q^{9} \\
& +5250816 q^{10}+\cdots, \\
Z_{0 ; 4}^{\text {inst }} & =-192 q^{4}-3328 q^{5}-32224 q^{6}-230400 q^{7}-1346944 q^{8}-6802432 q^{9} \\
& -30669248 q^{10}-\cdots, \\
Z_{0 ; 5}^{\text {inst }} & =960 q^{5}+21320 q^{6}+260320 q^{7}+2298680 q^{8}+16354800 q^{9}+99283840 q^{10}+\cdots
\end{aligned}
$$




\section{$E_{6}$ model}

$$
\begin{aligned}
Z_{0 ; 1}^{\text {inst }} & =3+27 q+81 q^{2}+255 q^{3}+702 q^{4}+1701 q^{5}+3930 q^{6}+8721 q^{7}+18225 q^{8} \\
& +37056 q^{9}+73116 q^{10}+\cdots, \\
Z_{0 ; 2}^{\text {inst }} & =-54 q^{2}-492 q^{3}-3078 q^{4}-14904 q^{5}-61320 q^{6}-224532 q^{7}-751788 q^{8} \\
& -2337264 q^{9}-6844338 q^{10}-\cdots, \\
Z_{0 ; 3}^{\text {inst }} & =243 q^{3}+4131 q^{4}+40095 q^{5}+287307 q^{6}+1683018 q^{7}+8515449 q^{8} \\
& +38457585 q^{9}+158463702 q^{10}+\cdots, \\
Z_{0 ; 4}^{\text {inst }} & =-1728 q^{4}-42120 q^{5}-559920 q^{6}-5344920 q^{7}-40835664 q^{8} \\
& -264772872 q^{9}-1510286688 q^{10}-\cdots, \\
Z_{0 ; 5}^{\text {inst }} & =15255 q^{5}+483585 q^{6}+8191530 q^{7}+97962210 q^{8}+925275420 q^{9} \\
& +7332946200 q^{10}+\cdots .
\end{aligned}
$$

\section{$\boldsymbol{E}_{7}$ model}

$$
\begin{aligned}
Z_{0 ; 1}^{\text {inst }} & =2+56 q+276 q^{2}+1360 q^{3}+4718 q^{4}+15960 q^{5}+46284 q^{6}+130064 q^{7} \\
& +334950 q^{8}+837872 q^{9}+1980756 q^{10}+\cdots \\
Z_{0 ; 2}^{\text {inst }} & =-272 q^{2}-4544 q^{3}-46416 q^{4}-335744 q^{5}-2008480 q^{6}-10255104 q^{7} \\
& -46868416 q^{8}-194576128 q^{9}-749189328 q^{10}+\cdots \\
Z_{0 ; 3}^{\text {inst }} & =3240 q^{3}+100134 q^{4}+1649088 q^{5}+18786852 q^{6}+168160176 q^{7} \\
& +1255563072 q^{8}+8154689040 q^{9}+47265867648 q^{10}+\cdots \\
Z_{0 ; 4}^{\text {inst }} & =-58432 q^{4}-2633088 q^{5}-60949696 q^{6}-960253440 q^{7} \\
& -11638833216 q^{8}-115871533568 q^{9}-988372855168 q^{10}-\cdots \\
Z_{0 ; 5}^{\text {inst }} & =1303840 q^{5}+77380260 q^{6}+2323737360 q^{7}+47046026140 q^{8} \\
& +724935311560 q^{9}+9088122264000 q^{10}+\cdots
\end{aligned}
$$




\section{$E_{8}$ model}

$$
\begin{aligned}
Z_{0 ; 1}^{\text {inst }} & =1+252 q+5130 q^{2}+54760 q^{3}+419895 q^{4}+2587788 q^{5}+13630694 q^{6} \\
& +63618120 q^{7}+269531955 q^{8}+1054198840 q^{9}+3854102058 q^{10}+\cdots, \\
Z_{0 ; 2}^{\text {inst }} & =-9252 q^{2}-673760 q^{3}-20534040 q^{4}-389320128 q^{5}-5398936120 q^{6} \\
& -59651033472 q^{7}-553157438400 q^{8}-4456706505600 q^{9} \\
& -31967377104276 q^{10}-\cdots \\
Z_{0 ; 3}^{\text {inst }} & =848628 q^{3}+115243155 q^{4}+6499779552 q^{5}+219488049810 q^{6} \\
& +5218126709400 q^{7}+95602979109024 q^{8}+1428776049708360 q^{9} \\
& +18102884896663488 q^{10}+\cdots, \\
Z_{0 ; 4}^{\text {inst }} & =-114265008 q^{4}-23064530112 q^{5}-1972983690880 q^{6}-100502238355200 q^{7} \\
& -3554323792345440 q^{8}-95341997143018752 q^{9} \\
& -2053905830285978880 q^{10}-\cdots \\
Z_{0 ; 5}^{\text {inst }} & =18958064400 q^{5}+5105167984850 q^{6}+594537323257800 q^{7} \\
& +41416214037843150 q^{8}+1996136210493389700 q^{9} \\
& +72464241398191308000 q^{10}+\cdots
\end{aligned}
$$

Note that for these principal series, $N_{0 ; n, n}^{\text {inst }}$ coincides with the genus zero, degree $n$ instanton number of the local $\boldsymbol{E}_{N}$ del Pezzo model computed in [23, 24].

\section{$\boldsymbol{E}_{0}$ model}

$$
\begin{aligned}
Z_{0 ; 1}^{\text {inst }} & =9+36 q+126 q^{2}+360 q^{3}+945 q^{4}+2268 q^{5}+5166 q^{6}+11160 q^{7} \\
& +23220 q^{8}+46620 q^{9}+90972 q^{10}+\cdots, \\
Z_{0 ; 2}^{\text {inst }} & =-18 q-252 q^{2}-1728 q^{3}-9000 q^{4}-38808 q^{5}-147384 q^{6}-506880 q^{7} \\
& -1613088 q^{8}-4813380 q^{9}-13609476 q^{10}-\cdots, \\
Z_{0 ; 3}^{\text {inst }} & =3 q+252 q^{2}+4158 q^{3}+40173 q^{4}+287415 q^{5}+1683450 q^{6}+8516418 q^{7} \\
& +38458233 q^{8}+158467806 q^{9}+605183100 q^{10}+\cdots, \\
Z_{0 ; 4}^{\text {inst }} & =-144 q^{2}-6048 q^{3}-107280 q^{4}-1235520 q^{5}-10796544 q^{6}-77538240 q^{7}
\end{aligned}
$$




$$
\begin{aligned}
& -479682720 q^{8}-2635776000 q^{9}-13140695232 q^{10}-\cdots \\
Z_{0 ; 5}^{\text {inst }} & =45 q^{2}+5670 q^{3}+189990 q^{4}+3508920 q^{5}+45151335 q^{6}+452510730 q^{7} \\
& +3763732545 q^{8}+27047637540 q^{9}+172619569800 q^{10}+\cdots
\end{aligned}
$$

We have checked that $\left\{N_{0 ; 3 n, n}^{\text {inst }}\right\}=\{3,-6,27,-192,1695, \cdots\}$ coincides with the genus zero, degree $n$ instanton number of the local $\mathbf{P}^{2}$ model $[23,24]$.

\section{$\boldsymbol{E}_{\tilde{1}}$ model}

$$
\begin{aligned}
Z_{0 ; 1}^{\text {inst }} & =8+16 q+56 q^{2}+112 q^{3}+280 q^{4}+528 q^{5}+1120 q^{6}+2016 q^{7}+3880 q^{8} \\
& +6720 q^{9}+12096 q^{10}+\cdots, \\
Z_{0 ; 2}^{\text {inst }} & =-4 q-56 q^{2}-280 q^{3}-1232 q^{4}-4212 q^{5}-13544 q^{6}-38584 q^{7}-105200 q^{8} \\
& -266696 q^{9}-653400 q^{10}-\cdots, \\
Z_{0 ; 3}^{\text {inst }} & =24 q^{2}+336 q^{3}+2688 q^{4}+15360 q^{5}+73584 q^{6}+303744 q^{7}+1137192 q^{8} \\
& +3897648 q^{9}+12515112 q^{10}+\cdots, \\
Z_{0 ; 4}^{\text {inst }} & =-4 q^{2}-224 q^{3}-3472 q^{4}-32704 q^{5}-232280 q^{6}-1351040 q^{7}-6818336 q^{8} \\
& -30695296 q^{9}-126302196 q^{10}-\cdots, \\
Z_{0 ; 5}^{\text {inst }} & =80 q^{3}+2800 q^{4}+44800 q^{5}+477160 q^{6}+3892240 q^{7}+26296560 q^{8} \\
& +153653920 q^{9}+800623600 q^{10}+\cdots .
\end{aligned}
$$

We have checked that $\left\{N_{0 ; 2 n, n}^{\mathrm{inst}}\right\}=\{-4,-4,-12,-48,-240,-1356,-8428, \cdots\}$ coincides with the genus zero, degree $n$ instanton number of the local $\mathbf{P}^{1} \times \mathbf{P}^{1}[6]$.

\section{Genus One Partition Functions}

\subsection{Genus one potentials}

In general, the determination of the genus one potential $F_{1}$ of a Calabi-Yau threefold $X$ requires the knowledge of the discriminant loci of the Picard-Fuchs system, which represent the singularities of the mirror complex moduli space $\mathcal{M}\left(X^{*}\right)$, and the identification of the power indices associated with each of the irreducible components of the discriminant loci $[2,16]$. 
For our case of the local $\boldsymbol{E}_{9}$ almost del Pezzo models, we find the following answers for the instanton parts:

$$
\begin{aligned}
\boldsymbol{E}_{0}: & F_{1}=\frac{1}{2} \log \left[\left(\frac{\left[\left(1+z_{1}\right)^{3}-z_{2}\right]}{\left(1-z_{2}\right)}\right)^{-\frac{1}{6}}\left(\frac{\partial z_{1}}{\partial \tilde{p}}\right)\right], \\
\boldsymbol{E}_{\tilde{1}}: & F_{1}=\frac{1}{2} \log \left[\left(\frac{\left[\left(1+z_{1}\right)^{2}-z_{2}\right]}{\left(1-z_{2}\right)}\right)^{-\frac{1}{6}}\left(1+z_{1}\right)^{-\frac{1}{3}}\left(\frac{\partial z_{1}}{\partial \tilde{p}}\right)\right], \\
\boldsymbol{E}_{5,6,7,8}: \quad F_{1} & =\frac{1}{2} \log \left[\left(\frac{\left(1-z_{2}+z_{1} z_{2}\right)}{\left(1-z_{2}\right)}\right)^{-\frac{1}{6}}\left(1-z_{1}\right)^{-\frac{9-N}{6}}\left(\frac{\partial z_{1}}{\partial \tilde{p}}\right)\right] .
\end{aligned}
$$

where $\tilde{p}:=p \psi(\tau)=\mathrm{e}^{2 \pi \mathrm{i} \sigma} \psi(\tau)$. The following identity will be used later:

$$
\left(1+\sum_{n=1}^{\infty} n c_{n}(\tau) z_{1}^{n}\right)\left(\frac{\partial z_{1}}{\partial \tilde{p}}\right)=\exp \left(-\sum_{n=1}^{\infty} c_{n}(\tau) z_{1}^{n}\right) .
$$

It would suffice here to list the first four terms of the $p$-expansions of $F_{1}$ :

$$
\begin{aligned}
F_{1}^{(0)} & =\frac{(y \tilde{p})}{4}\left(e_{2}+2\right)+\frac{(y \tilde{p})^{2}}{64}\left(5 e_{2}^{2}+8 e_{2}+3+\frac{8}{y}\right)+\frac{(y \tilde{p})^{3}}{1152}\left[39 e_{2}^{3}+54 e_{2}^{2}+e_{2}\left(117-\frac{8}{y}\right)\right. \\
& \left.+54+\frac{32}{y^{2}}\right]+\frac{(y \tilde{p})^{4}}{18432}\left[309 e_{2}^{4}+384 e_{2}^{3}+e_{2}^{2}\left(1746-\frac{784}{y}\right)-e_{2}\left(72-\frac{1120}{y}-\frac{64}{y^{2}}\right)\right. \\
& \left.+513-\frac{144}{y}+\frac{320}{y^{2}}\right], \\
F_{1}^{(\tilde{1})} & =\frac{(y \tilde{p})}{6}\left(e_{2}+3\right)+\frac{(y \tilde{p})^{2}}{144}\left[5 e_{2}^{2}+e_{2}\left(18-\frac{3}{y}\right)+16+\frac{8}{y}+\frac{4}{y^{2}}\right] \\
& +\frac{(y \tilde{p})^{3}}{1296}\left[13 e_{2}^{3}+e_{2}^{2}\left(57-\frac{15}{y}\right)+e_{2}\left(114-\frac{33}{y}+\frac{24}{y^{2}}\right)+88-\frac{20}{y^{3}}+\frac{84}{y^{2}}-\frac{24}{y}\right] \\
& +\frac{(y \tilde{p})^{4}}{31104}\left[103 e_{2}^{4}+e_{2}^{3}\left(540-\frac{174}{y}\right)+e_{2}^{2}\left(1584-\frac{864}{y}+\frac{324}{y^{2}}\right)\right. \\
& \left.+e_{2}\left(1936+\frac{1560}{y^{2}}-\frac{404}{y^{3}}-\frac{1176}{y}\right)+960-\frac{384}{y}+\frac{1854}{y^{2}}-\frac{966}{y^{3}}+\frac{291}{y^{4}}\right], \\
F_{1}^{(5)} & =\frac{y \tilde{p}}{12}\left(e_{2}+3\right)+\frac{(y \tilde{p})^{2}}{576}\left[5 e_{2}^{2}+e_{2}\left(18+\frac{6}{y}\right)+16+\frac{32}{y}+\frac{19}{y^{2}}\right] \\
& +\frac{(y \tilde{p})^{3}}{10368}\left[13 e_{2}^{3}+e_{2}^{2}\left(57+\frac{30}{y}\right)+e_{2}\left(114+\frac{138}{y}+\frac{87}{y^{2}}\right)+88+\frac{156}{y}+\frac{417}{y^{2}}+\frac{52}{y^{3}}\right] \\
& +\frac{(y \tilde{p})^{4}}{497664}\left[103 e_{2}^{4}+e_{2}^{3}\left(540+\frac{348}{y}\right)+e_{2}^{2}\left(1584+\frac{1656}{y}+\frac{1062}{y^{2}}\right)\right. \\
& \left.+e_{2}\left(1936+\frac{4152}{y}+\frac{6276}{y^{2}}+\frac{1252}{y^{3}}\right)+960+\frac{3648}{y}+\frac{9198}{y^{2}}+\frac{7836}{y^{3}}+\frac{921}{y^{4}}\right],
\end{aligned}
$$




$$
\begin{aligned}
F_{1}^{(6)} & =\frac{y \tilde{p}}{12}\left(e_{2}+2\right)+\frac{(y \tilde{p})^{2}}{576}\left[5 e_{2}^{2}+e_{2}\left(8+\frac{12}{y}\right)+3+\frac{28}{y}+\frac{16}{y^{2}}\right] \\
& +\frac{(y \tilde{p})^{3}}{31104}\left[39 e_{2}^{3}+e_{2}^{2}\left(54+\frac{180}{y}\right)+e_{2}\left(117+\frac{316}{y}+\frac{368}{y^{2}}\right)+54+\frac{216}{y}+\frac{992}{y^{2}}+\frac{256}{y^{3}}\right] \\
& +\frac{(y \tilde{p})^{4}}{1492992}\left[309 e_{2}^{4}+e_{2}^{3}\left(384+\frac{2088}{y}\right)+e_{2}^{2}\left(1746+\frac{3032}{y}+\frac{6112}{y^{2}}\right)\right. \\
& \left.+e_{2}\left(-72+\frac{8536}{y}+\frac{14496}{y^{2}}+\frac{8384}{y^{3}}\right)+513+\frac{2664}{y}+\frac{14720}{y^{2}}+\frac{25792}{y^{3}}+\frac{4608}{y^{4}}\right], \\
F_{1}^{(7)} & =\frac{y \tilde{p}}{12}\left(e_{2}+1\right)+\frac{(y \tilde{p})^{2}}{1152}\left[10 e_{2}^{2}-e_{2}\left(4-\frac{36}{y}\right)+\frac{27}{y}+\frac{27}{y^{2}}\right] \\
& +\frac{(y \tilde{p})^{3}}{20736}\left[26 e_{2}^{3}-e_{2}^{2}\left(42-\frac{180}{y}\right)+e_{2}\left(84-\frac{81}{y}+\frac{387}{y^{2}}\right)-32+\frac{99}{y}+\frac{315}{y^{2}}+\frac{216}{y^{3}}\right], \\
& +\frac{(y \tilde{p})^{4}}{3981312}\left[824 e_{2}^{4}-e_{2}^{3}\left(2272-\frac{8352}{y}\right)+e_{2}^{2}\left(6528-\frac{13176}{y}+\frac{30312}{y^{2}}\right)\right. \\
& \left.-e_{2}\left(9728-\frac{32112}{y}+\frac{8640}{y^{2}}-\frac{44496}{y^{3}}\right)+6016-\frac{18720}{y}+\frac{30627}{y^{2}}-\frac{39474}{y^{3}}+\frac{19683}{y^{4}}\right], \\
F_{1}^{(8)} & =\frac{y \tilde{p}}{12} e_{2}+\frac{(y \tilde{p})^{2}}{576}\left[5 e_{2}^{2}-e_{2}\left(12-\frac{24}{y}\right)+7-\frac{10}{y}+\frac{10}{y^{2}}\right] \\
+ & \frac{(y \tilde{p})^{3}}{31104}\left[39 e_{2}^{3}-e_{2}^{2}\left(180-\frac{360}{y}\right)+e_{2}\left(369-\frac{878}{y}+\frac{878}{y^{2}}\right)-276+\frac{712}{y}-\frac{480}{y}+\frac{320}{y^{3}}\right] \\
+ & \frac{(y \tilde{p})^{4}}{1492992}\left[309 e_{2}^{4}-e_{2}^{3}\left(2088-\frac{4176}{y}\right)+e_{2}^{2}\left(6858-\frac{18724}{y}+\frac{18724}{y^{2}}\right)\right. \\
& \left.-e_{2}\left(12336-\frac{39632}{y}+\frac{44880}{y^{2}}-\frac{29920}{y^{2}}\right)+9513-\frac{18180}{y^{3}}+\frac{9090}{y^{4}}\right],
\end{aligned}
$$

where we recall $\tilde{p}=p \psi(\tau)$. We see that the genus one Gromov-Witten partition function $Z_{1 ; n}$ takes the form predicted in (5.8), that is,

$$
Z_{1 ; n}=\left(Z_{0 ; 1}\right)^{n} \varpi^{2 n} \mathcal{P}_{1 ; n}\left(e_{2}, y^{-1}\right)
$$

where $\mathcal{P}_{1 ; n}$ is a degree $n$ polynomial in $e_{2}$ and $y^{-1}$.

We will give the partition functions only for the $\boldsymbol{E}_{7}$ and $\boldsymbol{E}_{8}$ models below.

\section{$E_{7}$ model}

$$
\begin{aligned}
& Z_{1 ; 1}^{(7)}=\frac{\chi}{24} A\left(E_{2}+A\right) \\
& Z_{1 ; 2}^{(7)}=\frac{\chi^{2}}{4608}\left(10 E_{2}^{2} A^{2}+36 E_{2} A B-4 E_{2} A^{3}+27 B^{2}+27 A^{2} B\right)
\end{aligned}
$$




$$
\begin{aligned}
Z_{1 ; 3}^{(7)} & =\frac{\chi^{3}}{165888}\left(26 E_{2}^{3} A^{3}-42 E_{2}^{2} A^{4}+180 E_{2}^{2} A^{2} B-81 E_{2} A^{3} B+387 E_{2} A B^{2}+84 E_{2} A^{5}\right. \\
& \left.+99 A^{4} B+216 B^{3}+315 B^{2} A^{2}-32 A^{6}\right), \\
Z_{1 ; 4}^{(7)} & =\frac{\chi^{4}}{63700992}\left(824 A^{4} E_{2}^{4}+8352 A^{3} E_{2}^{3} B-2272 A^{5} E_{2}^{3}+30312 A^{2} E_{2}^{2} B^{2}-13176 A^{4} E_{2}^{2} B\right. \\
& +6528 A^{6} E_{2}^{2}-9728 A^{7} E_{2}+44496 A E_{2} B^{3}-8640 A^{3} E_{2} B^{2}+32112 A^{5} E_{2} B+6016 A^{8} \\
& \left.+19683 B^{4}+30627 A^{4} B^{2}-18720 A^{6} B+39474 A^{2} B^{3}\right), \\
Z_{1 ; 5}^{(7)} & =\frac{\chi^{5}}{9555148800}\left(10970 A^{5} E_{2}^{5}+145800 A^{4} E_{2}^{4} B-42350 A^{6} E_{2}^{4}-1055250 A^{4} E_{2}^{2} B^{2}\right. \\
& +748350 A^{3} E_{2}^{3} B^{2}+151200 A^{7} E_{2}^{3}-345800 A^{8} E_{2}^{2}+1240650 A^{6} E_{2}^{2} B-389250 A^{5} E_{2}^{3} B \\
& +3230811 A^{5} E_{2} B^{2}-1961316 A^{7} B E_{2}+507584 A^{9} E_{2}+2008395 A E_{2} B^{4}-340032 A^{10} \\
& +1930635 A^{2} B^{4}+729000 B^{5}+2538540 A^{4} B^{3}-2195397 A^{6} B^{2}+1432080 A^{8} B \\
& \left.+1817100 A^{2} E_{2}^{2} B^{3}-208440 A^{3} E_{2} B^{3}\right) .
\end{aligned}
$$

\section{$\boldsymbol{E}_{8}$ model}

$$
\begin{aligned}
Z_{1 ; 1}^{(8)} & =\frac{1}{12 \varphi^{12}} E_{2} E_{4}, \\
Z_{1 ; 2}^{(8)} & =\frac{1}{1152 \varphi^{24}}\left(9 E_{4}^{3}+24 E_{2} E_{4} E_{6}+10 E_{2}^{2} E_{4}^{2}+5 E_{6}^{2}\right), \\
Z_{1 ; 3}^{(8)} & =\frac{1}{62208 \varphi^{36}}\left(472 E_{4}^{3} E_{6}+80 E_{6}^{3}+299 E_{2} E_{4}^{4}+439 E_{2} E_{4} E_{6}^{2}+360 E_{2}^{2} E_{4}^{2} E_{6}+78 E_{2}^{3} E_{4}^{3}\right), \\
Z_{1 ; 4}^{(8)} & =\frac{1}{11943936 \varphi^{48}}\left(37448 E_{2}^{2} E_{4}^{2} E_{6}^{2}+68768 E_{2} E_{4}^{4} E_{6}+29920 E_{2} E_{4} E_{6}^{3}+13809 E_{4}^{6}\right. \\
& \left.+57750 E_{4}^{3} E_{6}^{2}+17416 E_{2}^{2} E_{4}^{5}+4545 E_{6}^{6}+16704 E_{2}^{3} E_{4}^{3} E_{6}+2472 E_{2}^{4} E_{4}^{4}\right), \\
Z_{1 ; 5}^{(8)} & =\frac{1}{895795200 \varphi^{60}}\left(4102280 E_{2} E_{4}^{4} E_{6}^{2}+808765 E_{2} E_{4} E_{6}^{4}+1378600 E_{2}^{2} E_{4}^{2} E_{6}^{3}+103760 E_{6}^{5}\right. \\
& +2111000 E_{2}^{2} E_{4}^{5} E_{6}+951950 E_{2}^{3} E_{4}^{3} E_{6}^{2}+720057 E_{2} E_{4}^{7}+338950 E_{2}^{3} E_{4}^{6}+1749528 E_{4}^{4} E_{6} \\
& \left.+32910 E_{2}^{5} E_{4}^{5}+2340520 E_{4}^{3} E_{6}^{3}+291600 E_{2}^{4} E_{4}^{4} E_{6}\right) .
\end{aligned}
$$




\subsection{Modular anomaly equation}

To get the modular anomaly equation of genus one [18], we have only to notice that the genus one potential $F_{1}$ has $E_{2}(\tau)$-dependence both through $z_{1}$ and through $c_{n}(\tau)$, where we are considering $\left(\partial z_{1} / \partial \tilde{p}\right)$ as a function of $z_{1}$ and $c_{n}$ by (6.4).

The contribution of the former to the derivative $\left(\partial F_{1} / \partial E_{2}\right)$ is

$$
\left(\frac{\partial z_{1}}{\partial E_{2}}\right)\left(\frac{\partial F_{1}}{\partial z_{1}}\right)=\frac{1}{12 h}\left(\Theta_{p} z_{1}\right)\left(\Theta_{p} F_{1}\right)
$$

where we have used (5.5), while the latter

$$
-\frac{1}{2} \sum_{m=1}^{\infty}\left(\frac{\partial c_{m}}{\partial E_{2}}\right) \frac{\partial}{\partial c_{m}}\left(\sum_{n=1}^{\infty} c_{n} z_{1}^{n}+\log \left(1+\sum_{n=1}^{\infty} n c_{n} z_{1}^{n}\right)\right)=\frac{1}{24 h} \Theta_{p}\left(\Theta_{p}+1\right) F_{0} .
$$

Then we see that the anomaly equation for genus one takes the following form:

$$
h\left(\frac{\partial F_{1}}{\partial E_{2}}\right)=\frac{1}{12}\left(\Theta_{p} F_{0}\right)\left(\Theta_{p} F_{1}\right)+\frac{1}{24} \Theta_{p}\left(\Theta_{p}+1\right) F_{0},
$$

which can be rewritten in terms of the Gromov-Witten partition functions as

$$
h \frac{\partial Z_{1 ; n}(\tau)}{\partial E_{2}(\tau)}=\frac{1}{24} \sum_{h=0}^{1} \sum_{k=1}^{n-1} k(n-k) Z_{h ; k}(\tau) Z_{1-h ; n-k}(\tau)+\frac{1}{24} n(n+1) Z_{0 ; n}(\tau),
$$

which takes the from just predicted in (2.17).

\subsection{Elliptic instanton numbers}

Let $N_{1 ; n, m}^{\mathrm{inst}} \in \mathbf{Z}$ be the genus one instanton number of bidegree $(n, m)$, and

$$
Z_{1 ; n}^{\text {inst }}(\tau)=\sum_{m=0}^{\infty} N_{1 ; n, m}^{\text {inst }} q^{m}
$$

be its generating function. According to [2], we have the following decomposition of the genus one Gromov-Witten partition function:

$$
Z_{1 ; n}(\tau)=\sum_{k \mid n}\left(\sigma_{-1}(k) Z_{1 ; \frac{n}{k}}^{\text {inst }}(k \tau)+\frac{1}{12} k^{-1} Z_{0 ; \frac{n}{k}}^{\text {inst }}(k \tau)\right) .
$$

The inversion of this equation is given by

$$
Z_{1 ; n}^{\text {inst }}(\tau)=\sum_{k \mid n}\left(a_{-1}(k) Z_{1 ; \frac{n}{k}}(k \tau)-\frac{1}{12} a_{-3}(k) Z_{0 ; \frac{n}{k}}(k \tau)\right),
$$

where we have introduced the arithmetic functions $a_{l}(n):=\sum_{m \mid n} \mu(m) \mu(n / m) m^{l}$.

We give the generating functions of the genus one instanton numbers for each model. 


\section{$\boldsymbol{E}_{5}$ model}

$$
\begin{aligned}
Z_{1 ; 1}^{\text {inst }} & =-8 q^{4}-32 q^{5}-80 q^{6}-192 q^{7}-464 q^{8}-1024 q^{9}-2080 q^{10}-\cdots \\
Z_{1 ; 2}^{\text {inst }} & =18 q^{4}+192 q^{5}+1040 q^{6}+4352 q^{7}+15752 q^{8}+51328 q^{9}+153448 q^{10}+\cdots \\
Z_{1 ; 3}^{\text {inst }} & =-16 q^{4}-384 q^{5}-3920 q^{6}-26848 q^{7}-145440 q^{8}-671936 q^{9} \\
& -2754816 q^{10}-\cdots \\
Z_{1 ; 4}^{\text {inst }} & =5 q^{4}+320 q^{5}+6320 q^{6}+71168 q^{7}+577264 q^{8}+3758848 q^{9}+20853184 q^{10}+\cdots \\
Z_{1 ; 5}^{\text {inst }} & =-96 q^{5}-4640 q^{6}-93056 q^{7}-1170496 q^{8}-10922336 q^{9}-82513280 q^{10}-\cdots
\end{aligned}
$$

\section{$\boldsymbol{E}_{6}$ model}

$$
\begin{aligned}
Z_{1 ; 1}^{\text {inst }} & =-6 q^{3}-54 q^{4}-162 q^{5}-528 q^{6}-1566 q^{7}-3888 q^{8}-9414 q^{9}-21870 q^{10}-\cdots \\
Z_{1 ; 2}^{\text {inst }} & =9 q^{3}+243 q^{4}+2322 q^{5}+13824 q^{6}+68283 q^{7}+290466 q^{8}+1094580 q^{9} \\
& +3785940 q^{10}+\cdots, \\
Z_{1 ; 3}^{\text {inst }} & =-4 q^{3}-324 q^{4}-7290 q^{5}-85458 q^{6}-700164 q^{7}-4599990 q^{8}-25682910 q^{9} \\
& -126394182 q^{10}-\cdots, \\
Z_{1 ; 4}^{\text {inst }} & =135 q^{4}+8262 q^{5}+194532 q^{6}+2729754 q^{7}+27756027 q^{8}+226001070 q^{9} \\
& +1557055332 q^{10}+\cdots, \\
Z_{1 ; 5}^{\text {inst }} & =-3132 q^{5}-185346 q^{6}-4812210 q^{7}-78689502 q^{8}-948813714 q^{9} \\
& -9183023298 q^{10}-\cdots .
\end{aligned}
$$

\section{$\boldsymbol{E}_{7}$ model}

$$
\begin{aligned}
Z_{1 ; 1}^{\text {inst }} & =-4 q^{2}-112 q^{3}-564 q^{4}-3056 q^{5}-11108 q^{6}-40528 q^{7}-123112 q^{8} \\
& -367552 q^{9}-989236 q^{10}-\cdots \\
Z_{1 ; 2}^{\text {inst }} & =3 q^{2}+336 q^{3}+9018 q^{4}+101088 q^{5}+862098 q^{6}+5657664 q^{7}+32067860 q^{8} \\
& +158512832 q^{9}+712084479 q^{10}+\cdots,
\end{aligned}
$$




$$
\begin{aligned}
Z_{1 ; 3}^{\text {inst }} & =-224 q^{3}-20496 q^{4}-640032 q^{5}-10716104 q^{6}-128761968 q^{7}-1208615256 q^{8} \\
& -9504050688 q^{9}-64763400720 q^{10}-\cdots \\
Z_{1 ; 4}^{\text {inst }} & =12042 q^{4}+1116896 q^{5}+41444664 q^{6}+903550592 q^{7}+14095889180 q^{8} \\
& +172098048640 q^{9}+1743551210128 q^{10}+\cdots \\
Z_{1 ; 5}^{\text {inst }} & =-574896 q^{5}-57707124 q^{6}-2511634800 q^{7}-66979775872 q^{8} \\
& -1286028782768 q^{9}-19346827285068 q^{10}-\cdots
\end{aligned}
$$

\section{$E_{8}$ model}

$$
\begin{aligned}
Z_{1 ; 1}^{\text {inst }} & =-2 q-510 q^{2}-11780 q^{3}-142330 q^{4}-1212930 q^{5}-8207894 q^{6}-46981540 q^{7} \\
& -236385540 q^{8}-1072489860 q^{9}-4467531670 q^{10}-\cdots, \\
Z_{1 ; 2}^{\text {inst }} & =762 q^{2}+205320 q^{3}+11361870 q^{4}+317469648 q^{5}+5863932540 q^{6} \\
& +81295293600 q^{7}+909465990330 q^{8}+8597134346400 q^{9} \\
& +70867771453026 q^{10}+\cdots \\
Z_{1 ; 3}^{\text {inst }} & =-246788 q^{3}-76854240 q^{4}-6912918432 q^{5}-323516238180 q^{6} \\
& -9882453271500 q^{7}-2218762317666600 q^{8}-3933705832711600 q^{9} \\
& -57747806496416088 q^{10}-\cdots \\
Z_{1 ; 4}^{\text {inst }} & =76413073 q^{4}+27863327760 q^{5}+3478600115600 q^{6}+234196316814400 q^{7} \\
& +10330930335961770 q^{8}+332747064864457152 q^{9} \\
& +8378290954495817152 q^{10}+\cdots \\
Z_{1 ; 5}^{\text {inst }} & =-23436186174 q^{5}-9930641443350 q^{6}-1585090167772500 q^{7} \\
& -140688512133882000 q^{8}-8255877490179586950 q^{9} \\
& -353737948953627859770 q^{10}-\cdots
\end{aligned}
$$

We see that for the principal series, $N_{1 ; n, n}^{\text {inst }}$ coincides with the genus one, degree $n$ instanton number of the $E_{N}$ del Pezzo model first obtained in [24]. Genus one instanton numbers for the $E_{8}$ model have been computed in [23]. 


\section{$\boldsymbol{E}_{0}$ model}

$$
\begin{aligned}
Z_{1 ; 1}^{\text {inst }} & =-18 q^{3}-72 q^{4}-252 q^{5}-774 q^{6}-2106 q^{7}-5292 q^{8}-12564 q^{9} \\
& -28278 q^{10}-\cdots, \\
Z_{1 ; 2}^{\text {inst }} & =108 q^{3}+1152 q^{4}+7812 q^{5}+41022 q^{6}+181656 q^{7}+710856 q^{8}+2526516 q^{9} \\
& +8310492 q^{10}+\cdots, \\
Z_{1 ; 3}^{\text {inst }} & =-336 q^{3}-7368 q^{4}-85284 q^{5}-700896 q^{6}-4602090 q^{7}-25679052 q^{8} \\
& -126406392 q^{9}-562694940 q^{10}-\cdots, \\
Z_{1 ; 4}^{\text {inst }} & =630 q^{3}+26343 q^{4}+496404 q^{5}+6119388 q^{6}+57190644 q^{7}+437749110 q^{8} \\
& +2875241088 q^{9}+16711846956 q^{10}+\cdots, \\
Z_{1 ; 5}^{\text {inst }} & =-756 q^{3}-59976 q^{4}-1817298 q^{5}-33012216 q^{6}-430550244 q^{7} \\
& -4429221912 q^{8}-38028172446 q^{9}-282776491026 q^{10}-\cdots
\end{aligned}
$$

We have checked that $\left\{N_{1 ; 3 n, n}^{\text {inst }}\right\}=\{0,0,-10,231,-4452, \cdots\}$ coincides with the genus one, degree $n$ instanton number of the $\mathbf{P}^{2}$ model first obtained in [24].

\section{$\boldsymbol{E}_{\tilde{1}}$ model}

$$
\begin{aligned}
Z_{1 ; 1}^{\text {inst }} & =-16 q^{4}-32 q^{5}-112 q^{6}-224 q^{7}-608 q^{8}-1152 q^{9}-2576 q^{10}-\cdots \\
Z_{1 ; 2}^{\text {inst }} & =84 q^{4}+424 q^{5}+2264 q^{6}+8176 q^{7}+29364 q^{8}+88416 q^{9} \\
& +260360 q^{10}+\cdots, \\
Z_{1 ; 3}^{\text {inst }} & =-224 q^{4}-2208 q^{5}-17392 q^{6}-95872 q^{7}-467376 q^{8}-1947008 q^{9} \\
& -7471488 q^{10}-\cdots, \\
Z_{1 ; 4}^{\text {inst }} & =350 q^{4}+6272 q^{5}+72512 q^{6}+576704 q^{7}+3778068 q^{8}+20848384 q^{9} \\
& +102392928 q^{10}+\cdots, \\
Z_{1 ; 5}^{\text {inst }} & =-336 q^{4}-10976 q^{5}-188880 q^{6}-2130016 q^{7}-18652816 q^{8}-134027488 q^{9} \\
& -833043952 q^{10}-\cdots .
\end{aligned}
$$

We have checked that $\left\{N_{1 ; 2 n, n}^{\text {inst }}\right\}=\{0,0,0,9,136,1616,17560, \cdots\}$ coincides with the genus one, degree $n$ instanton number of the $\mathbf{P}^{1} \times \mathbf{P}^{1}$ model listed in [6]. 


\section{Higher Genus Partition Functions}

In contrast to the genus zero or genus one case, we cannot evaluate directly the higher genus Gromov-Witten partition functions [3]. However, the modular anomaly equation (2.17) invented in [18] is so powerful that it determines the partition function $Z_{g ; n}^{(N)}(\tau)$ up to finite constants.

\subsection{Partition functions as modular forms}

In this subsection, we propose a conjecture on the form of the partition functions $Z_{g ; n}(\tau)$ of the six models in terms of the modular forms.

First we define $\chi^{(N)}$ for each of the six models by

$$
\begin{aligned}
& \chi^{(0)}(\tau)=\frac{9 q^{\frac{1}{6}}}{\eta(\tau)^{4}}, \quad \chi^{(\tilde{1})}(\tau)=\frac{8 q^{\frac{1}{4}}}{\eta(\tau)^{2} \eta(2 \tau)^{2}}, \quad \chi^{(5)}(\tau)=\frac{4 q^{\frac{1}{2}} \eta(2 \tau)^{4}}{\eta(\tau)^{4} \eta(4 \tau)^{4}}, \\
& \chi^{(6)}(\tau)=\frac{3 q^{\frac{1}{2}}}{\eta(\tau)^{3} \eta(3 \tau)^{3}}, \quad \chi^{(7)}(\tau)=\frac{2 q^{\frac{1}{2}}}{\eta(\tau)^{4} \eta(2 \tau)^{4}}, \quad \chi^{(8)}(\tau)=\frac{q^{\frac{1}{2}}}{\eta(\tau)^{12}} .
\end{aligned}
$$

Then we propose the conjectured forms of the partition functions of them:

$$
\begin{aligned}
& Z_{g ; n}^{(0)}(\tau)=\left(\chi^{(0)}(\tau)\right)^{n} P_{2 g-2+2 n}^{(0)}\left(E_{2}(\tau), \varpi^{(6)}(\tau), H(\tau)\right), \\
& Z_{g ; n}^{(\tilde{1})}(\tau)=\left(\chi^{(\tilde{1})}(\tau)\right)^{n} P_{2 g-2+2 n}^{(\tilde{1})}\left(E_{2}(\tau), \vartheta_{3}(2 \tau)^{4}, \vartheta_{4}(2 \tau)^{4}\right), \\
& Z_{g ; n}^{(5)}(\tau)=\left(\chi^{(5)}(\tau)\right)^{n} P_{2 g-2+2 n}^{(5)}\left(E_{2}(\tau), \vartheta_{3}(2 \tau)^{4}, \vartheta_{4}(2 \tau)^{4}\right), \\
& Z_{g ; n}^{(6)}(\tau)=\left(\chi^{(6)}(\tau)\right)^{n} P_{2 g-2+3 n}^{(6)}\left(E_{2}(\tau), \varpi^{(6)}(\tau), H(\tau)\right), \\
& Z_{g ; n}^{(7)}(\tau)=\left(\chi^{(7)}(\tau)\right)^{n} P_{2 g-2+4 n}^{(7)}\left(E_{2}(\tau), A(\tau), B(\tau)\right), \\
& Z_{g ; n}^{(8)}(\tau)=\left(\chi^{(8)}(\tau)\right)^{n} P_{2 g-2+6 n}^{(8)}\left(E_{2}(\tau), E_{4}(\tau), E_{6}(\tau)\right),
\end{aligned}
$$

where each $P^{(N)}$ is a polynomial over $\mathbf{Q}$ in three variables the subscript of which shows its weight as a quasi-modular form.

$\boldsymbol{E}_{7}$ model We list the genus two partition functions of the $\boldsymbol{E}_{7}$ model.

$$
\begin{aligned}
& Z_{2 ; 1}^{(7)}=\frac{\chi}{5760} A\left(6 A^{2}+3 B+5 E_{2}^{2}+10 A E_{2}\right), \\
& Z_{2 ; 2}^{(7)}=\frac{\chi^{2}}{1658880}\left(-64 A^{5}+999 A^{3} B+2349 A B^{2}+190 E_{2}^{3} A^{2}+30 E_{2}^{2} A^{3}+810 E_{2}^{2} A B\right.
\end{aligned}
$$




$$
\begin{aligned}
& \left.+132 A^{4} E_{2}+1251 A^{2} B E_{2}+1215 E_{2} B^{2}\right), \\
Z_{2 ; 3}^{(7)} & =\frac{\chi^{3}}{318504960}\left(10561 A^{7}-27183 A^{5} B+222723 A^{3} B^{2}+273699 A B^{3}+4600 E_{2}^{4} A^{3}\right. \\
& -5920 E_{2}^{3} A^{4}+36480 E_{2}^{3} A^{2} B-288 E_{2}^{2} A^{3} B+105840 E_{2}^{2} A B^{2}+20544 E_{2}^{2} A^{5} \\
& \left.+200988 E_{2} B^{2} A^{2}-12100 E_{2} A^{6}+61704 E_{2} A^{4} B+103680 E_{2} B^{3}\right), \\
Z_{2 ; 4}^{(7)} & =\frac{\chi^{4}}{61152952320}\left(-1622467 A^{9}+6147828 A^{7} B-5446746 A^{5} B^{2}+33187428 A^{3} B^{3}\right. \\
& +26235333 A B^{4}+108800 A^{4} E_{2}^{5}+1236480 E_{2}^{4} A^{3} B-280320 E_{2}^{4} A^{5}+7149024 E_{2}^{2} A^{5} B \\
& +5454720 E_{2}^{3} B^{2} A^{2}+1067008 E_{2}^{3} A^{6}-1639296 E_{2}^{3} A^{4} B+1937232 E_{2}^{2} A^{3} B^{2} \\
& -2044976 E_{2}^{2} A^{7}+10817280 E_{2}^{2} A B^{3}+18498600 E_{2} A^{4} B^{2}-8131560 E_{2} A^{6} B \\
& \left.+24054408 E_{2} A^{2} B^{3}+2625048 E_{2} A^{8}+8048160 E_{2} B^{4}\right), \\
Z_{2 ; 5}^{(7)} & =\frac{\chi^{5}}{17612050268160}\left(-142044480 E_{2}^{4} A^{5} B+621872640 E_{2}^{3} A^{6} B-342771840 E_{2}^{3} A^{4} B^{2}\right. \\
& +355628304 E_{2}^{2} A^{9}+55837440 E_{2}^{5} A^{4} B+332170560 E_{2}^{4} A^{3} B^{2}+991837440 E_{2}^{3} A^{2} B^{3} \\
& +873400320 E_{2} B^{5}-1325802096 E_{2}^{2} A^{7} B+727775280 E_{2}^{2} A^{3} B^{3}+1478062080 E_{2}^{2} A B^{4} \\
& -462511536 E_{2} A^{10}+2359788336 E_{2}^{2} A^{5} B^{2}-2810004192 E_{2} A^{6} B^{2}+292244077 A^{11} \\
& +3887980560 E_{2} A^{2} B^{4}+4762800000 E_{2} A^{4} B^{3}+1930802688 E_{2} A^{8} B+3804160 A^{5} E_{2}^{6} \\
& -1325620701 A^{9} B+2471168610 A^{7} B^{2}-1132668090 A^{5} B^{3}+6150153825 A^{3} B^{4} \\
& \left.+3302730855 A B^{5}-14400000 E_{2}^{5} A^{6}+62142720 E_{2}^{4} A^{7}-172019840 E_{2}^{3} A^{8}\right) .
\end{aligned}
$$

$\boldsymbol{E}_{8}$ model We give the partition functions of genus up to five.

$$
\begin{aligned}
Z_{2 ; 1}^{(8)} & =\frac{1}{1440 \varphi^{12}} E_{4}\left(E_{4}+5 E_{2}^{2}\right), \\
Z_{2 ; 2}^{(8)} & =\frac{1}{207360 \varphi^{24}}\left(417 E_{2} E_{4}^{3}+190 E_{4}^{2} E_{2}^{3}+540 E_{2}^{2} E_{4} E_{6}+225 E_{2} E_{6}^{2}+356 E_{4}^{2} E_{6}\right), \\
Z_{2 ; 3}^{(8)} & =\frac{1}{2488320 \varphi^{36}}\left(575 E_{2}^{4} E_{4}^{3}+3040 E_{2}^{3} E_{4}^{2} E_{6}+4690 E_{2}^{2} E_{4} E_{6}^{2}+3548 E_{2}^{2} E_{4}^{4}+1600 E_{6}^{3} E_{2}\right. \\
& \left.+10176 E_{6} E_{4}^{3} E_{2}+2231 E_{4}^{5}+5244 E_{4}^{2} E_{6}^{2}\right), \\
Z_{2 ; 4}^{(8)} & =\frac{1}{179159040 \varphi^{48}}\left(77280 E_{2}^{4} E_{6} E_{4}^{3}+209200 E_{2}^{2} E_{6}^{3} E_{4}+547760 E_{2}^{2} E_{6} E_{4}^{4}+214811 E_{4}^{6} E_{2}\right. \\
& +203900 E_{2}^{3} E_{6}^{2} E_{4}^{2}+103252 E_{4}^{5} E_{2}^{3}+827230 E_{6}^{2} E_{4}^{3} E_{2}+10200 E_{2}^{5} E_{4}^{4}+57375 E_{6}^{4} E_{2}
\end{aligned}
$$




$$
\begin{aligned}
& \left.+420616 E_{4}^{5} E_{6}+314360 E_{4}^{2} E_{6}^{3}\right) \\
& Z_{2 ; 5}^{(8)}=\frac{1}{12899450880 \varphi^{60}}\left(15422230 E_{6}^{4} E_{4}^{2}+43101209 E_{6}^{2} E_{4}^{5}+5522085 E_{4}^{8}+1903680 E_{6}^{5} E_{2}\right. \\
& +18947800 E_{2}^{3} E_{4}^{5} E_{6}+1744920 E_{2}^{5} E_{4}^{4} E_{6}+50040570 E_{2}^{2} E_{6}^{2} E_{4}^{4}+6480025 E_{2}^{4} E_{4}^{3} E_{6}^{2} \\
& +11149400 E_{2}^{3} E_{4}^{2} E_{6}^{3}+8437860 E_{2}^{2} E_{4} E_{6}^{4}+51231560 E_{6}^{3} E_{4}^{3} E_{2}+42541168 E_{4}^{6} E_{6} E_{2} \\
& \left.+2482715 E_{4}^{6} E_{2}^{4}+9555018 E_{4}^{7} E_{2}^{2}+178320 E_{2}^{6} E_{4}^{5}\right), \\
& Z_{3 ; 1}^{(8)}=\frac{1}{362880 \varphi^{12}} E_{4}\left(4 E_{6}+21 E_{2} E_{4}+35 E_{2}^{3}\right), \\
& Z_{3 ; 2}^{(8)}=\frac{1}{34836480 \varphi^{24}}\left(14984 E_{4}^{2} E_{6} E_{2}+8925 E_{2}^{2} E_{4}^{3}+2275 E_{4}^{2} E_{2}^{4}+7560 E_{2}^{3} E_{4} E_{6}+4725 E_{2}^{2} E_{6}^{2}\right. \\
& \left.+3540 E_{4}^{4}+4071 E_{4} E_{6}^{2}\right) \\
& Z_{3 ; 3}^{(8)}=\frac{1}{209018880 \varphi^{36}}\left(138104 E_{4}^{4} E_{6}+224024 E_{6} E_{4}^{3} E_{2}^{2}+36400 E_{2}^{4} E_{4}^{2} E_{6}+224456 E_{4}^{2} E_{6}^{2} E_{2}\right. \\
& +49584 E_{4} E_{6}^{3}+68460 E_{2}^{3} E_{4} E_{6}^{2}+55006 E_{2}^{3} E_{4}^{4}+6055 E_{2}^{5} E_{4}^{3}+97431 E_{4}^{5} E_{2} \\
& \left.+33600 E_{6}^{3} E_{2}^{2}\right) \\
& Z_{3 ; 4}^{(8)}=\frac{1}{90296156160 \varphi^{48}}\left(28134630 E_{4}^{7}+151049093 E_{4}^{4} E_{6}^{2}+25488295 E_{4} E_{6}^{4}+966630 E_{2}^{6} E_{4}^{4}\right. \\
& +189296376 E_{6}^{2} E_{4}^{3} E_{2}^{2}+8172360 E_{2}^{5} E_{6} E_{4}^{3}+31388000 E_{2}^{3} E_{6}^{3} E_{4}+88718416 E_{2}^{3} E_{6} E_{4}^{4} \\
& +24977155 E_{2}^{4} E_{6}^{2} E_{4}^{2}+13366787 E_{4}^{5} E_{2}^{4}+12119625 E_{6}^{4} E_{2}^{2}+137926976 E_{4}^{2} E_{6}^{3} E_{2} \\
& \left.+51557313 E_{4}^{6} E_{2}^{2}+192353224 E_{4}^{5} E_{6} E_{2}\right), \\
& Z_{3 ; 5}^{(8)}=\frac{1}{1083553873920 \varphi^{60}}\left(274848600 E_{6}^{5} E_{4}+2868277704 E_{6}^{3} E_{4}^{4}+1662616800 E_{6} E_{4}^{7}\right. \\
& +635585864 E_{2}^{4} E_{4}^{5} E_{6}+323470350 E_{2}^{3} E_{4} E_{6}^{4}+349176520 E_{2}^{4} E_{4}^{2} E_{6}^{3}+41643000 E_{2}^{6} E_{4}^{4} E_{6} \\
& +2109910578 E_{2}^{3} E_{6}^{2} E_{4}^{4}+174368705 E_{2}^{5} E_{4}^{3} E_{6}^{2}+3866100 E_{2}^{7} E_{4}^{5}+101077200 E_{6}^{5} E_{2}^{2} \\
& +424873884 E_{4}^{7} E_{2}^{3}+1739056502 E_{6}^{4} E_{4}^{2} E_{2}+5180110741 E_{6}^{2} E_{4}^{5} E_{2}+70310947 E_{4}^{6} E_{2}^{5} \\
& \left.+3045375184 E_{6}^{3} E_{4}^{3} E_{2}^{2}+2693483096 E_{4}^{6} E_{6} E_{2}^{2}+696828225 E_{4}^{8} E_{2}\right), \\
& Z_{4 ; 1}^{(8)}=\frac{1}{87091200 \varphi^{12}} E_{4}\left(39 E_{4}^{2}+80 E_{2} E_{6}+210 E_{2}^{2} E_{4}+175 E_{2}^{4}\right), \\
& Z_{4 ; 2}^{(8)}=\frac{1}{2090188800 \varphi^{24}}\left(53220 E_{2} E_{4}^{4}+112540 E_{2}^{2} E_{4}^{2} E_{6}+45185 E_{2}^{3} E_{4}^{3}+7385 E_{4}^{2} E_{2}^{5}\right. \\
& \left.+28350 E_{2}^{4} E_{4} E_{6}+23625 E_{2}^{3} E_{6}^{2}+61065 E_{2} E_{4} E_{6}^{2}+6300 E_{6}^{6}+49402 E_{6} E_{4}^{3}\right), \\
& Z_{4 ; 3}^{(8)}=\frac{1}{75246796800 \varphi^{36}}\left(3164700 E_{2}^{4} E_{4} E_{6}^{2}+8993259 E_{4}^{5} E_{2}^{2}+14111840 E_{6}^{2} E_{4}^{3}+806400 E_{6}^{4}\right.
\end{aligned}
$$




$$
\begin{aligned}
& +25171632 E_{2} E_{6} E_{4}^{4}+13855280 E_{2}^{3} E_{6} E_{4}^{3}+8963520 E_{2} E_{6}^{3} E_{4}+20453520 E_{2}^{2} E_{6}^{2} E_{4}^{2} \\
& \left.+4014627 E_{4}^{6}+208985 E_{2}^{6} E_{4}^{3}+2016000 E_{6}^{3} E_{2}^{3}+1417920 E_{2}^{5} E_{4}^{2} E_{6}+2638125 E_{2}^{4} E_{4}^{4}\right), \\
& Z_{4 ; 4}^{(8)}=\frac{1}{5417769369600 \varphi^{48}}\left(3336940980 E_{2}^{3} E_{4}^{3} E_{6}^{2}+7817234620 E_{2} E_{6}^{2} E_{4}^{4}+3248768730 E_{6}^{3} E_{4}^{3}\right. \\
& +5085796952 E_{2}^{2} E_{4}^{5} E_{6}+101280375 E_{6}^{5}+3550525000 E_{2}^{2} E_{4}^{2} E_{6}^{3}+1290318725 E_{2} E_{4} E_{6}^{4} \\
& +936363912 E_{4}^{6} E_{2}^{3}+1481276055 E_{4}^{7} E_{2}+2912603799 E_{4}^{6} E_{6}+1216807640 E_{2}^{4} E_{4}^{4} E_{6} \\
& +152620090 E_{2}^{5} E_{4}^{5}+78676080 E_{2}^{6} E_{6} E_{4}^{3}+410158000 E_{2}^{4} E_{6}^{3} E_{4}+274844990 E_{2}^{5} E_{6}^{2} E_{4}^{2} \\
& \left.+8381520 E_{2}^{7} E_{4}^{4}+202702500 E_{6}^{4} E_{2}^{3}\right), \\
& Z_{4 ; 5}^{(8)}=\frac{1}{52010585948160 \varphi^{60}}\left(16869986640 E_{6}^{5} E_{4} E_{2}+8944068536 E_{2}^{5} E_{4}^{5} E_{6}+1167070464 E_{6}^{6}\right. \\
& +2035152000 E_{6}^{5} E_{2}^{3}+436442160 E_{2}^{7} E_{4}^{4} E_{6}+854577430 E_{4}^{6} E_{2}^{6}+114133172104 E_{6}^{2} E_{4}^{4} \\
& +183172864792 E_{6}^{3} E_{4}^{4} E_{2}+36942885 E_{2}^{8} E_{4}^{5}+5146355025 E_{2}^{4} E_{4} E_{6}^{4}+11890359900 E_{4}^{9} \\
& +7455500881 E_{4}^{7} E_{2}^{4}+23616142080 E_{4}^{8} E_{2}^{2}+60902666801 E_{6}^{4} E_{4}^{3}+2043907670 E_{2}^{6} E_{4}^{3} E_{6}^{2} \\
& +4688369560 E_{2}^{5} E_{4}^{2} E_{6}^{3}+54769592870 E_{6}^{4} E_{4}^{2} E_{2}^{2}+66152468720 E_{6}^{3} E_{4}^{3} E_{2}^{3} \\
& +60955175392 E_{4}^{6} E_{6} E_{2}^{3}+109420106696 E_{6} E_{4}^{7} E_{2}+170157797734 E_{6}^{2} E_{4}^{5} E_{2}^{2} \\
& \left.+35736239660 E_{2}^{4} E_{6}^{2} E_{4}^{4}\right) \\
& Z_{5 ; 1}^{(8)}=\frac{1}{11496038400 \varphi^{12}} E_{4}\left(136 E_{4} E_{6}+429 E_{4}^{2} E_{2}+440 E_{2}^{2} E_{6}+770 E_{2}^{3} E_{4}+385 E_{2}^{5}\right), \\
& Z_{5 ; 2}^{(8)}=\frac{1}{3310859059200 \varphi^{24}}\left(4510275 E_{2}^{2} E_{4}^{3}+10553400 E_{2}^{2} E_{4}^{4}+2494800 E_{6}^{3} E_{2}+3358995 E_{4}^{5}\right. \\
& +14869360 E_{2}^{3} E_{4}^{2} E_{6}+12090870 E_{2}^{2} E_{4} E_{6}^{2}+19568568 E_{6} E_{4}^{3} E_{2}+2245320 E_{2}^{5} E_{4} E_{6} \\
& \left.+7083727 E_{4}^{2} E_{6}^{2}+512050 E_{4}^{2} E_{2}^{6}+2338875 E_{2}^{2} E_{6}^{2}\right), \\
& Z_{5 ; 3}^{(8)}=\frac{1}{9932577177600 \varphi^{36}}\left(935093824 E_{6}^{2} E_{4}^{3} E_{2}+233170300 E_{2}^{4} E_{6} E_{4}^{3}+296640960 E_{2}^{2} E_{6}^{3} E_{4}\right. \\
& +837550728 E_{2}^{2} E_{6} E_{4}^{4}+453680480 E_{2}^{3} E_{6}^{2} E_{4}^{2}+16385600 E_{2}^{6} E_{4}^{2} E_{6}+42513240 E_{2}^{5} E_{4} E_{6}^{2} \\
& +201151929 E_{4}^{5} E_{2}^{3}+36275085 E_{2}^{5} E_{4}^{4}+53222400 E_{6}^{4} E_{2}+266767491 E_{4}^{6} E_{2} \\
& \left.+405268284 E_{4}^{5} E_{6}+268326944 E_{4}^{2} E_{6}^{3}+33264000 E_{6}^{3} E_{2}^{4}+2155615 E_{2}^{7} E_{4}^{3}\right), \\
& Z_{5 ; 4}^{(8)}=\frac{1}{2860582227148800 \varphi^{48}}\left(12207942670 E_{2}^{6} E_{4}^{5}+523849095 E_{2}^{8} E_{4}^{4}+156150752805 E_{4}^{8}\right. \\
& +113811930320 E_{2}^{5} E_{4}^{4} E_{6}+1311485716360 E_{4}^{6} E_{6} E_{2}+1760563778482 E_{2}^{2} E_{6}^{2} E_{4}^{4} \\
& +286289201000 E_{2}^{2} E_{4} E_{6}^{4}+381058740370 E_{2}^{4} E_{4}^{3} E_{6}^{2}+1449394307792 E_{6}^{3} E_{4}^{3} E_{2}
\end{aligned}
$$




$$
\begin{aligned}
& +1106487740990 E_{6}^{2} E_{4}^{5}+44575839000 E_{6}^{5} E_{2}+109025587484 E_{4}^{6} E_{2}^{4} \\
& +774483173328 E_{2}^{3} E_{4}^{5} E_{6}+531170439360 E_{2}^{3} E_{4}^{2} E_{6}^{3}+5431290480 E_{2}^{7} E_{6} E_{4}^{3} \\
& +37160939200 E_{2}^{5} E_{6}^{3} E_{4}+337421738130 E_{4}^{7} E_{2}^{2}+21439577390 E_{2}^{6} E_{6}^{2} E_{4}^{2} \\
& \left.+22344052500 E_{6}^{4} E_{2}^{4}+344998537324 E_{6}^{4} E_{4}^{2}\right), \\
Z_{5 ; 5}^{(8)} & =\frac{1}{102980960177356800 \varphi^{60}}\left(31511006810584 E_{6}^{5} E_{4}^{2}+177751951656248 E_{6}^{3} E_{4}^{5}\right. \\
& +78175349827680 E_{6} E_{4}^{8}+344664297670 E_{4}^{6} E_{2}^{7}+21179704043952 E_{4}^{8} E_{2}^{3} \\
& +4104656416113 E_{4}^{7} E_{2}^{5}+41030103891064 E_{4}^{6} E_{6} E_{2}^{4}+1264302270000 E_{6}^{5} E_{2}^{4} \\
& +2891093990400 E_{6}^{6} E_{2}+31336414684620 E_{4}^{9} E_{2}+155081412353885 E_{6}^{4} E_{4}^{3} E_{2} \\
& +296146234031236 E_{6}^{2} E_{4}^{6} E_{2}+43310617469240 E_{6}^{3} E_{4}^{3} E_{2}^{4}+19202491494120 E_{2}^{5} E_{6}^{2} E_{4}^{4} \\
& +46735606475470 E_{6}^{4} E_{4}^{2} E_{2}^{3}+2649529315125 E_{2}^{5} E_{4} E_{6}^{4}+154506124080 E_{2}^{8} E_{4}^{4} E_{6} \\
& +803244450470 E_{2}^{7} E_{4}^{3} E_{6}^{2}+4107192009800 E_{2}^{6} E_{4}^{5} E_{6}+2083500320440 E_{2}^{6} E_{4}^{2} E_{6}^{3} \\
& +149437965048686 E_{6}^{2} E_{4}^{5} E_{2}^{3}+21211745049000 E_{6}^{5} E_{4} E_{2}^{2}+144355295784864 E_{6} E_{4}^{7} E_{2}^{2} \\
& \left.+11970104685 E_{2}^{9} E_{4}^{5}+236773842080568 E_{6}^{3} E_{4}^{4} E_{2}^{2}\right) .
\end{aligned}
$$

\subsection{Gopakumar-Vafa invariants}

We can extract from the higher genus Gromov-Witten partition function $Z_{g ; n}$ an important integer-invariants, the Gopakumar-Vafa invariants [13], which we will explain very briefly. For more details on this subject, see [18, 4, 19, 21].

Let us first consider a BPS state in M theory compactified on a Calabi-Yau threefold $X$ which is realized by the M2-brane wrapped around a holomorphic curve $C$. In addition to the Abelian gauge charge corresponding to the homology class $[C] \in H_{2}(X)$, such a state carries also a quantum number of the $5 \mathrm{D}$ little group $S O(4)=S U(2)_{L} \times S U(2)_{R}$.

To clarify the origin of the Lorentz quantum number, let $\mathcal{M}_{\beta}$ be the moduli space of curves in $X$ with a fixed homology class $\beta \in H_{2}(X)$, and $\pi: \hat{\mathcal{M}}_{\beta} \rightarrow \mathcal{M}_{\beta}$ the extended moduli space with its Jacobian fibration, which means that $\hat{\mathcal{M}}_{\beta}$ parametrizes all the pairs of a curve of the fixed homology class $\beta$ and a flat line bundle on it. Namely, $\hat{\mathcal{M}}_{\beta}$ is the appropriate moduli space for a BPS M2 brane with its Abelian charge fixed. 
The BPS states for this degree of freedom arise from quantization of the moduli space, that is, the cohomology group $H^{*}\left(\hat{\mathcal{M}}_{\beta} ; \mathbf{C}\right)$ represented by the harmonic differential forms. The $S U(2)_{L}$ and $S U(2)_{R}$ Lorentz quantum numbers come from the Lefshetz $S U(2)$ actions for the fibre and base direction of the Jacobian fibration $\pi: \hat{\mathcal{M}}_{\beta} \rightarrow \mathcal{M}_{\beta}$ respectively.

We take the following representation of the Lorentz spin content of the BPS states with fixed $\beta$ :

$$
H^{*}\left(\hat{\mathcal{M}}_{\beta} ; \mathbf{C}\right)=\sum_{h=0}^{g} I_{h} \otimes U_{h ; \beta}, \quad I_{h}:=\left[V_{1 / 2}^{L} \oplus 2 V_{0}^{L}\right]^{\otimes h},
$$

where $g$ is the maximum value of the genus that a curve of the fixed homology class $\beta$ can have, and $V_{j}$ the irreducible $S U(2)$ module of spin $j$. Let $U_{h ; \beta}=\oplus_{j} N_{h, j ; \beta} V_{j}^{R}$ be the irreducible decomposition of the $S U(2)_{R}$ module above. Then $N_{h, j ; \beta} \in \mathbf{Z}_{\geq 0}$ is the multiplicity of the BPS states with the $S O(4)$ Lorentz quantum number $I_{h+1} \otimes V_{j}^{R}$ and the Abelian gauge charge $\beta \in H_{2}(X)$.

The Gopakumar-Vafa invariant $N_{h ; \beta}^{\mathrm{GV}}$ with fixed $h \in \mathbf{Z}$ and $\beta \in H_{2}(X)$ is then given by the index with respect to the $S U(2)_{R}$ on $U_{h ; \beta}$, that is, $N_{h ; \beta}^{\mathrm{GV}}:=\sum_{j} \mathrm{e}^{2 \pi \mathrm{i} j}(2 j+1) N_{h, j ; \beta}$.

It has been found in [13] that the instanton part of the full partition function of IIA topological string on $X[3]$ can be obtained by

$$
\sum_{g=0}^{\infty} x^{2 g-2} F_{g}=\sum_{\beta \in H_{2}(X)}^{\prime} \sum_{h=0}^{\infty} \sum_{m=1}^{\infty} N_{h ; \beta}^{\mathrm{GV}} \frac{1}{m}\left[2 \sin \left(\frac{m x}{2}\right)\right]^{2 h-2} \mathrm{e}^{2 \pi \mathrm{i} m\langle J, \beta\rangle},
$$

where $J \in H^{2}(X ; \mathbf{C})$ is the complexified Kähler class of $X$.

From now on we turn to the investigation of the Gopakumar-Vafa invariants of one of the six local two-parameter models of the $\boldsymbol{E}_{9}$ almost del Pezzo surface. First let $N_{g ; n, m}^{\mathrm{GV}}$ be the Gopakumar-Vafa invariant of genus $g$ and bidegree $(n, m)$, and define its generating function by

$$
Z_{g ; n}^{\mathrm{GV}}(\tau)=\sum_{m=0}^{\infty} N_{g ; n, m}^{\mathrm{GV}} q^{m}
$$

We can show from (7.8) that the partition function of the genus $g$ Gromov-Witten invariants $Z_{g ; n}(\tau)$ admits the following decomposition into the generating functions of Gopakumar-Vafa invariants of genus $h \leq g$ :

$$
Z_{g ; n}(\tau)=\sum_{h=0}^{g} \beta_{g, h} \sum_{k \mid n} k^{2 g-3} Z_{h ; \frac{n}{k}}^{\mathrm{GV}}(k \tau)
$$


where $\beta_{g, h}$ is the rational number defined by the following expansion:

$$
\left(\frac{\sin (x / 2)}{(x / 2)}\right)^{2 h-2}=\sum_{g=h}^{\infty} \beta_{g, h} x^{2(g-h)} .
$$

Note that $\beta_{g, 0}$ coincides with the one given earlier in (2.22).

Now we give the Möbius inversion formula of (7.10) following [4, Prop.2.1]. To this end, let us first define the rational number $\alpha_{g, h}$ by

$$
\left(\frac{\arcsin (x / 2)}{(x / 2)}\right)^{2 h-2}=\sum_{g=h}^{\infty} \alpha_{g, h} x^{2(g-h)} .
$$

The Möbius inversion for the Gopakumar-Vafa invariants can then be written as

$$
Z_{g ; n}^{\mathrm{GV}}(\tau)=\sum_{h=0}^{g} \alpha_{g, h} \sum_{k \mid n} \mu(k) k^{2 h-3} Z_{h ; \frac{n}{k}}(k \tau) .
$$

Let us take the $\boldsymbol{E}_{8}$ model and substitute the leading term (2.22) of the partition function in (7.13). Then we see for each $(g, n) \neq(0,1)$,

$$
Z_{g ; n}^{\mathrm{GV}(8)}(\tau)=\sum_{h=0}^{g} \alpha_{g, h} \beta_{h, 0} n^{2 h-3} \sum_{k \mid n} \mu(k)+O\left(q^{n}\right)=O\left(q^{n}\right) .
$$

To be more explicit, we describe below the decompositions of the Gromov-Witten partition functions $Z_{g ; n}(\tau)$ into the generating functions of Gopakumar-Vafa invariants $Z_{g ; n}^{\mathrm{GV}}(\tau)(7.10)$ and their Möbius inversions (7.13) for lower genera.

Genus zero For the genus zero case, (7.10) and (7.13) read

$$
Z_{0 ; n}(\tau)=\sum_{k \mid n} k^{-3} Z_{0 ; \frac{n}{k}}^{\mathrm{GV}}(k \tau), \quad Z_{0 ; n}^{\mathrm{GV}}(\tau)=\sum_{k \mid n} \mu(k) k^{-3} Z_{0 ; \frac{n}{k}}(k \tau),
$$

which shows that $Z_{0 ; n}^{\text {inst }}(\tau)=Z_{0 ; n}^{\mathrm{GV}}(\tau)$, that is, the genus zero Gopakumar-Vafa invariants are nothing but the numbers of rational instantons.

Genus one For the genus one cases, we have

$$
\begin{aligned}
& Z_{1 ; n}(\tau)=\sum_{k \mid n} k^{-1}\left(Z_{1 ; \frac{n}{k}}^{\mathrm{GV}}(k \tau)+\frac{1}{12} Z_{0 ; \frac{n}{k}}^{\mathrm{GV}}(k \tau)\right), \\
& Z_{1 ; n}^{\mathrm{GV}}(\tau)=\sum_{k \mid n} \mu(k)\left(k^{-1} Z_{1 ; \frac{n}{k}}(k \tau)-\frac{1}{12} k^{-3} Z_{0 ; \frac{n}{k}}(k \tau)\right) .
\end{aligned}
$$


The transformation formulae between $Z_{1 ; n}^{\mathrm{GV}}$ and $Z_{1 ; n}^{\mathrm{inst}}$ are

$$
Z_{1 ; n}^{\mathrm{GV}}(\tau)=\sum_{k \mid n} Z_{1 ; \frac{n}{k}}^{\mathrm{inst}}(k \tau), \quad Z_{1 ; n}^{\mathrm{inst}}(\tau)=\sum_{k \mid n} \mu(k) Z_{1 ; \frac{n}{k}}^{\mathrm{GV}}(k \tau)
$$

Genus two For the genus two case,

$$
\begin{aligned}
& Z_{2 ; n}(\tau)=\sum_{k \mid n} k\left(Z_{2 ; \frac{n}{k}}^{\mathrm{GV}}(k \tau)+\frac{1}{240} Z_{0 ; \frac{n}{k}}^{\mathrm{GV}}(k \tau)\right), \\
& Z_{2 ; n}^{\mathrm{GV}}(\tau)=\sum_{k \mid n} \mu(k)\left(k Z_{2 ; \frac{n}{k}}(k \tau)-\frac{1}{240} k^{-3} Z_{0 ; \frac{n}{k}}(k \tau)\right) .
\end{aligned}
$$

On the other hand, we have also the genus two instanton numbers $N_{2 ; n, m}^{\text {inst }}[3,(7.7)]$, the generating function of which $Z_{2 ; n}^{\text {inst }}(\tau)=\sum_{m=0}^{\infty} N_{2 ; n, m}^{\text {inst }} q^{m}$ is defined through

$$
Z_{2 ; n}(\tau)=Z_{2 ; n}^{\text {inst }}(\tau)+\frac{1}{240} \sum_{k \mid n} k Z_{0 ; \frac{n}{k}}^{\text {inst }}(k \tau) .
$$

We see that the two partition functions $Z_{2 ; n}^{\mathrm{GV}}(\tau)$ and $Z_{2 ; n}^{\text {inst }}(\tau)$ are related each other by

$$
Z_{2 ; n}^{\mathrm{inst}}(\tau)=\sum_{k \mid n} k Z_{2 ; \frac{n}{k}}^{\mathrm{GV}}(k \tau), \quad Z_{2 ; n}^{\mathrm{GV}}(\tau)=\sum_{k \mid n} \mu(k) k Z_{2 ; \frac{n}{k}}^{\mathrm{inst}}(k \tau)
$$

Genus three Finally for the genus three case,

$$
\begin{aligned}
& Z_{3 ; n}(\tau)=\sum_{k \mid n} k^{3}\left(Z_{3 ; \frac{n}{k}}^{\mathrm{GV}}(k \tau)-\frac{1}{12} Z_{2 ; \frac{n}{k}}^{\mathrm{GV}}(k \tau)+\frac{1}{6048} Z_{0 ; \frac{n}{k}}^{\mathrm{GV}}(k \tau)\right), \\
& Z_{3 ; n}^{\mathrm{GV}}(\tau)=\sum_{k \mid n} \mu(k)\left(k^{3} Z_{3 ; \frac{n}{k}}(k \tau)+\frac{1}{12} k Z_{2 ; \frac{n}{k}}(k \tau)-\frac{31}{60480} k^{-3} Z_{0 ; \frac{n}{k}}(k \tau)\right) .
\end{aligned}
$$

The formula (7.13) enables us to convert the Gromov-Witten partition function $Z_{g ; n}(\tau)$ to the generating function of the Gopakumar-Vafa invariants $Z_{g ; n}^{\mathrm{GV}}(\tau)$. Let $N_{g ; n}^{\mathrm{GV}}\left(B_{N}\right)$ be the Gopakumar-Vafa invariant of the local $\boldsymbol{E}_{N}$ del Pezzo model of genus $g$ and degree $n$. Based on the calculation of several $Z_{g ; n}^{\mathrm{GV}}(\tau)$ using (7.13), we propose the following conjecture for the Gopakumar-Vafa invariants of the local $\boldsymbol{E}_{9}$ del Pezzo models:

$$
\begin{array}{ll}
\boldsymbol{E}_{0}: & N_{g ; 3 n, n}^{\mathrm{GV}}=N_{g ; n}^{\mathrm{GV}}\left(\mathbf{P}^{2}\right), \\
\boldsymbol{E}_{1}: & N_{g ; 2 n, n}^{\mathrm{GV}}=N_{g ; n}^{\mathrm{GV}}\left(\mathbf{P}^{1} \times \mathbf{P}^{1}\right), \\
\boldsymbol{E}_{N}: & N_{g ; n, n}^{\mathrm{GV}}=N_{g ; n}^{\mathrm{GV}}\left(B_{N}\right), \quad N=5,6,7,8 .
\end{array}
$$


It should be noted that the evaluation of the left hand side is much easier than that of the right hand side [21]. We will show some examples of $Z_{g ; n}^{\mathrm{GV}}(\tau)$ below to see the integrality of their $q$-expansions.

$\boldsymbol{E}_{7}$ model We give the genus two Gopakumar-Vafa generating functions.

$$
\begin{aligned}
Z_{2 ; 1}^{\mathrm{GV}} & =6 q^{4}+168 q^{5}+860 q^{6}+4976 q^{7}+18660 q^{8}+72160 q^{9}+226952 q^{10} \\
& +712128 q^{11}+\cdots, \\
Z_{2 ; 2}^{\mathrm{GV}} & =-580 q^{4}-12224 q^{5}-171192 q^{6}-1520960 q^{7}-11191692 q^{8}-67475456 q^{9} \\
& -361410816 q^{10}-\cdots, \\
Z_{2 ; 3}^{\mathrm{GV}} & =986 q^{4}+90952 q^{5}+2505136 q^{6}+43815752 q^{7}+539969082 q^{8} \\
& +5314601592 q^{9}+43546643132 q^{10}+\cdots, \\
Z_{2 ; 4}^{\mathrm{GV}} & =-844 q^{4}-219392 q^{5}-14554008 q^{6}-456217600 q^{7}-9386376248 q^{8} \\
& -142590577280 q^{9}-1733995192624 q^{10}-\cdots, \\
Z_{2 ; 5}^{\mathrm{GV}} & =116880 q^{5}+22288580 q^{6}+1484462912 q^{7}+53446857696 q^{8} \\
& +1298602990944 q^{9}+23677762683308 q^{10}+\cdots .
\end{aligned}
$$

$\boldsymbol{E}_{8}$ model We give only the genus two and three cases.

$$
\begin{aligned}
Z_{2 ; 1}^{\mathrm{GV}} & =3 q^{2}+772 q^{3}+19467 q^{4}+257796 q^{5}+2391067 q^{6}+17484012 q^{7}+107445366 q^{8} \\
& +577157904 q^{9}+2782194327 q^{10}+\cdots, \\
Z_{2 ; 2}^{\mathrm{GV}} & =-4 q^{2}-25604 q^{3}-3075138 q^{4}-135430120 q^{5}-3449998524 q^{6}-61300761264 q^{7} \\
& -839145842528 q^{8}-9401698267600 q^{9}-89741934231984 q^{10}-\cdots, \\
Z_{2 ; 3}^{\mathrm{GV}} & =30464 q^{3}+26356767 q^{4}+4012587684 q^{5}+267561063651 q^{6}+10669237946340 q^{7} \\
& +296540296415919 q^{8}+6281046300189120 q^{9}+107386914608369634 q^{10}+\cdots, \\
Z_{2 ; 4}^{\mathrm{GV}} & =-26631112 q^{4}-18669096840 q^{5}-3493725635712 q^{6}-315335792669280 q^{7} \\
& -17502072462748056 q^{8}-680822976267281568 q^{9}-20119222969453708672 q^{10} \\
& -476723960943969692160 q^{11}-\cdots,
\end{aligned}
$$




$$
\begin{aligned}
Z_{2 ; 5}^{\mathrm{GV}} & =16150498760 q^{5}+11074858711765 q^{6}+2457788116576020 q^{7} \\
& +280285943363605460 q^{8}+20134110289153178480 q^{9} \\
& +1021994028815246670450 q^{10}+\cdots \\
Z_{3 ; 1}^{\mathrm{GV}} & =-4 q^{3}-1038 q^{4}-28200 q^{5}-403530 q^{6}-4027020 q^{7}-31528152 q^{8} \\
& -206468416 q^{9}-1176822312 q^{10}-\cdots \\
Z_{3 ; 2}^{\mathrm{GV}} & =1296 q^{3}+494144 q^{4}+38004700 q^{5}+1400424188 q^{6}+32782202520 q^{7} \\
& +559061195716 q^{8}+7518370093000 q^{9}+83886353406048 q^{10} \\
& +804126968489640 q^{11}+\cdots \\
Z_{3 ; 3}^{\mathrm{GV}} & =-1548 q^{3}-5707354 q^{4}-1607880090 q^{5}-158684891624 q^{6}-8435743979080 q^{7} \\
& -294159368706504 q^{8}-7512935612951670 q^{9}-150615781749573158 q^{10} \\
& -2483798853495519960 q^{11}-\cdots, \\
Z_{3 ; 4}^{\mathrm{GV}} & =5889840 q^{4}+8744913564 q^{5}+2548788575530 q^{6}+314635716180400 q^{7} \\
& +22243167756986804 q^{8}+1053665475134158016 q^{9}+36762786441521664780 q^{10} \\
& +1005501515252382449280 q^{11}+\cdots, \\
Z_{3 ; 5}^{\mathrm{GV}} & =-7785768630 q^{5}-8996745286730 q^{6}-2835031032258700 q^{7} \\
& -420624614518458350 q^{8}-37292995978411176810 q^{9} \\
& -2255647477866896285790 q^{10}-101168121676653460498460 q^{11}-\cdots
\end{aligned}
$$

\subsection{Partition functions as Jacobi forms}

The solution (2.20) of the modular anomaly equation (2.17) implies that the partition function $Z_{g ; n}(\tau \mid \mu)$ is completely determined only if we could fix the anomaly-free part of its numerator (2.10), $T_{g ; n}^{0}(\tau \mid \mu)$, which is an $\boldsymbol{E}_{8}$ Weyl-invariant Jacobi form of weight $2 g-2+6 n$ and index $n$. We introduce here some notation: let $\mathcal{J}_{k, n}^{\Gamma}\left(\boldsymbol{E}_{8}\right)$ be the space of the $\boldsymbol{E}_{8}$ Weyl-invariant Jacobi forms of weight $k$ and index $n$ for a modular group $\Gamma \subset S L(2 ; \mathbf{Z})$; $\mathcal{J}_{*, *}^{\Gamma}\left(\boldsymbol{E}_{8}\right):=\bigoplus_{k, n} \mathcal{J}_{k, n}^{\Gamma}\left(\boldsymbol{E}_{8}\right)$ the total space of such forms, which has a structure of a graded $M_{*}(\Gamma)$-algebra. Unfortunately, we do not have the generators of $\mathcal{J}_{*, *}^{S L(2 ; \mathbf{Z})}\left(\boldsymbol{E}_{8}\right)$ [41] to fix 
$T_{g ; n}^{0}(\tau \mid \mu)$ up to finite unknown coefficients.

However a powerful method to generate certain elements of $\mathcal{J}_{*, *}^{S L(2 ; \mathbf{Z})}\left(\boldsymbol{E}_{8}\right)$ from the theta function $\Theta_{\boldsymbol{E}_{8}}$ has been used to obtain $T_{0 ; n}^{0}(\tau \mid \mu)$ for $n=2,3,4$ in [27].

We will now explain the method. First, we note that $\Theta_{\boldsymbol{E}_{8}}^{(n)}(\tau \mid \mu):=\Theta_{\boldsymbol{E}_{8}}(n \tau \mid n \mu)$ is an element of $\mathcal{J}_{4, n}^{\Gamma_{0}(n)}\left(\boldsymbol{E}_{8}\right)$. Secondly, the slash action of $\gamma \in S L(2 ; \mathbf{Z})$ on $G(\tau \mid \mu) \in \mathcal{J}_{k, m}^{\Gamma_{0}(n)}\left(\boldsymbol{E}_{8}\right)$ is defined by

$$
(G \mid \gamma)(\tau \mid \mu):=\frac{1}{(c \tau+d)^{k}} \exp \left[-\frac{\pi \mathrm{i} m c}{c \tau+d}(\mu \mid \mu)\right] G\left(\frac{a \tau+b}{c \tau+d} \mid \frac{\mu}{c \tau+d}\right), \quad \gamma=\left(\begin{array}{ll}
a & b \\
c & d
\end{array}\right),
$$

which satisfies $\left(G \mid \gamma_{1}\right)\left|\gamma_{2}=G\right|\left(\gamma_{1} \gamma_{2}\right)$. Note that $G \mid \gamma=G$ for any $\gamma \in \Gamma_{0}(n)$ by definition, and $G \in \mathcal{J}_{k, m}^{S L(2 ; \mathbf{Z})}\left(\boldsymbol{E}_{8}\right)$ if and only if $G \mid \gamma=G$ for any $\gamma \in S L(2 ; \mathbf{Z})$. Thirdly, consider the coset space $\Gamma_{0}(n) \backslash S L(2 ; \mathbf{Z})$, on which $S L(2 ; \mathbf{Z})$ acts as permutation from the right, and the cardinality of which is $c(n):=n \prod_{p \mid n}\left(1+p^{-1}\right)$; in particular we can take $\left\{I, T, T S, \ldots, T S^{p-1}\right\}$ as its representatives if $n=p$ is prime [37]. Then for $f(\tau) \in M_{k}\left(\Gamma_{0}(n)\right)$, we define $\sigma_{a}^{(n)}(f)(\tau \mid \mu)$ by

$$
\sum_{a=0}^{c(n)} t^{c(n)-a} \sigma_{a}^{(n)}(f)=\prod_{\gamma \in \Gamma_{0}(n) \backslash S L(2 ; \mathbf{Z})}\left[t+\left(f \Theta_{\mathbf{E}_{8}}^{(n)} \mid \gamma\right)\right],
$$

that is, $\sigma_{a}^{(n)}(f)$ is the ath basic symmetric polynomial in $\left\{\left(f \Theta_{\boldsymbol{E}_{8}}^{(n)} \mid \gamma\right)\right\}$. From the argument above, we can see that $\sigma_{a}^{(n)}(f) \in \mathcal{J}_{a(k+4), a n}^{S L(2 ; \mathbf{Z})}\left(\boldsymbol{E}_{8}\right)$. Note in particular that $\sigma_{1}^{(n)}(1)$ is the $n$th Hecke transform of $\Theta_{\boldsymbol{E}_{8}}$. More generally, we can see that any permutation invariant combination gives an element of $\mathcal{J}_{*, *}^{S L(2 ; \mathbf{Z})}\left(\boldsymbol{E}_{8}\right)$; for example, if $f_{i} \in M_{k_{i}}\left(\Gamma_{0}(n)\right)$ for $i=1,2$, then $\sum_{\gamma}\left(f_{1} \Theta_{\boldsymbol{E}_{8}}^{(n)} \mid \gamma\right) \cdot\left(f_{2} \Theta_{\boldsymbol{E}_{8}}^{(n)} \mid \gamma\right)$ is an element of $\mathcal{J}_{8+k_{1}+k_{2}, 2 n}^{S L(2 ; \mathbf{Z})}\left(\boldsymbol{E}_{8}\right)$, and so on.

We will now determine doubly winding partition functions $T_{g ; 2}^{0}(\tau \mid \mu) \in \mathcal{J}_{2 g+10,2}^{S L(2 ; \mathbf{Z})}\left(\boldsymbol{E}_{8}\right)$ for lower $g$ s based on the assumption that they are obtained by the procedure that we have just explained above. We need to consider only $\sigma_{1}^{(2)}: M_{*}\left(\Gamma_{0}(2)\right) \rightarrow \mathcal{J}_{*+4,2}^{S L(2 ; \mathbf{Z})}\left(\boldsymbol{E}_{8}\right)$, which is a homomorphism of $M_{*}(S L(2 ; \mathbf{Z}))$-modules. It turns out that $T_{g ; 2}^{0}$ should be found in the free $M_{*}(S L(2 ; \mathbf{Z}))$-module generated by the three elements $\sigma_{1}^{(2)}(A B), \sigma_{1}^{(2)}\left(B^{2}\right)$ and $\sigma_{1}^{(2)}(B)=\left(\Theta_{\boldsymbol{E}_{8}}\right)^{2}$; we do not use $\sigma_{1}^{(2)}(1)$ and $\sigma_{1}^{(2)}(A)$ as generators because the $q$-expansion 
of them specialized to the four $\boldsymbol{E}_{N \neq 7,8}$ models has a pole; for $\boldsymbol{E}_{5}$ model, for example,

$$
\begin{aligned}
\sigma_{1}^{(2)}(1)\left(4 \tau \mid \tau \omega_{5}\right) & =2\left(\frac{\Theta_{\boldsymbol{E}_{8}}\left(4 \tau \mid \tau \omega_{5}\right)}{\vartheta_{2}(2 \tau)^{4} \vartheta_{3}(2 \tau)^{2}}\right)^{2}\left(9 \vartheta_{3}(2 \tau)^{4}+\vartheta_{4}(2 \tau)^{4}\right) \\
\sigma_{1}^{(2)}(A)\left(4 \tau \mid \tau \omega_{5}\right) & =-\frac{1}{4}\left(\frac{\Theta_{\boldsymbol{E}_{8}}\left(4 \tau \mid \tau \omega_{5}\right)}{\vartheta_{2}(2 \tau)^{4} \vartheta_{3}(2 \tau)^{2}}\right)^{2}\left(15 \vartheta_{3}(2 \tau)^{8}+26 \vartheta_{4}(2 \tau)^{4} \vartheta_{3}(2 \tau)^{4}-\vartheta_{4}(2 \tau)^{8}\right)
\end{aligned}
$$

Incidentally, $A B$ and $B^{2}$ can be expanded as

$$
A B=\frac{1}{3}\left(E_{4} A+E_{6}\right), \quad B^{2}=\frac{1}{9}\left(4 E_{6} A+3 E_{4} B+2 E_{4}^{2}\right) .
$$

We list the result of our fitting including the fundamental result of $T_{0 ; 2}^{0}$ in [27]:

$$
\begin{aligned}
T_{0 ; 2}^{0} & =\frac{1}{12} \sigma_{1}^{(2)}(A B), \\
T_{1 ; 2}^{0} & =\frac{1}{576}\left[E_{4}\left(\Theta_{\boldsymbol{E}_{8}}\right)^{2}+6 \sigma_{1}^{(2)}\left(B^{2}\right)\right] \\
T_{2 ; 2}^{0} & =\frac{1}{51840}\left[26 E_{6}\left(\Theta_{\boldsymbol{E}_{8}}\right)^{2}+63 E_{4} \sigma_{1}^{(2)}(A B)\right] \\
T_{3 ; 2}^{0} & =\frac{1}{11612160}\left[445 E_{4}^{2}\left(\Theta_{\boldsymbol{E}_{8}}\right)^{2}+832 E_{6} \sigma_{1}^{(2)}(A B)+1260 E_{4} \sigma_{1}^{(2)}\left(B^{2}\right)\right], \\
T_{4 ; 2}^{0} & =\frac{1}{1045094400}\left[6692 E_{4} E_{6}\left(\Theta_{\boldsymbol{E}_{8}}\right)^{2}+13599 E_{4}^{2} \sigma_{1}^{(2)}(A B)+7560 E_{6} \sigma_{1}^{(2)}\left(B^{2}\right)\right] \\
T_{5 ; 2}^{0} & =\frac{1}{1655429529600}\left[\left(603615 E_{4}^{3}+523520 E_{6}^{2}\right)\left(\Theta_{\boldsymbol{E}_{8}}\right)^{2}+2249856 E_{4} E_{6} \sigma_{1}^{(2)}(A B)\right. \\
& \left.+1844370 E_{4}^{2} \sigma_{1}^{(2)}\left(B^{2}\right)\right], \\
T_{6 ; 2}^{0} & =\frac{1}{1506440871936000}\left[123290398 E_{4}^{2} E_{6}\left(\Theta_{\boldsymbol{E}_{8}}\right)^{2}\right. \\
& \left.+\left(185440941 E_{4}^{3}+61328640 E_{6}^{2}\right) \sigma_{1}^{(2)}(A B)+180540360 E_{4} E_{6} \sigma_{1}^{(2)}\left(B^{2}\right)\right], \\
T_{7 ; 2}^{0} & =\frac{1}{867709942235136000}\left[\left(2926360905 E_{4}^{4}+5095068160 E_{4} E_{6}^{2}\right)\left(\Theta_{\boldsymbol{E}_{8}}\right)^{2}\right. \\
& \left.+16042720896 E_{4}^{2} E_{6} \sigma_{1}^{(2)}(A B)+\left(8745249240 E_{4}^{3}+3358817280 E_{6}^{2}\right) \sigma_{1}^{(2)}\left(B^{2}\right)\right] .
\end{aligned}
$$

It is then easy to recover $Z_{g ; 2}(\tau \mid \mu)$ by the solution $(2.21)$ of the modular anomaly equation. As a consistency check of our procedure, we can look into the integrality of the Gopakumar-Vafa invariants; indeed for the $\boldsymbol{E}_{7}$ model, we have

$$
\begin{aligned}
& Z_{3 ; 2}^{\mathrm{GV}(7)}(\tau)=5 q^{4}+560 q^{5}+18350 q^{6}+240736 q^{7}+2479193 q^{8}+\cdots \\
& Z_{4 ; 2}^{\mathrm{GV}(7)}(\tau)=-896 q^{6}-21248 q^{7}-354032 q^{8}-3578624 q^{9}-30445968 q^{10}+\cdots
\end{aligned}
$$




$$
\begin{aligned}
& Z_{5 ; 2}^{\mathrm{GV}(7)}(\tau)=7 q^{6}+784 q^{7}+30124 q^{8}+443392 q^{9}+5276873 q^{10}+\cdots, \\
& Z_{6 ; 2}^{\mathrm{GV}(7)}(\tau)=-1228 q^{8}-32064 q^{9}-617904 q^{10}-6946048 q^{11}-66942248 q^{12}-\cdots, \\
& Z_{7 ; 2}^{\mathrm{GV}(7)}(\tau)=9 q^{8}+1008 q^{9}+44450 q^{10}+720928 q^{11}+9741094 q^{12}+\cdots .
\end{aligned}
$$

We also find the triply winding partition function in the same manner:

$$
\begin{aligned}
& T_{0 ; 3}^{0}=\frac{1}{864}\left[20 \sigma_{1}^{(3)}\left(H^{4}\right)+972 \eta^{24} \sigma_{1}^{(3)}(1)-3 E_{4}\left(\Theta_{\boldsymbol{E}_{8}}\right)^{3}\right] \\
& T_{1 ; 3}^{0}=\frac{1}{2592}\left[24 \sigma_{1}^{(3)}\left(H^{4}\left(\varpi^{(6)}\right)^{2}\right)-E_{6}\left(\Theta_{\boldsymbol{E}_{8}}\right)^{3}\right]
\end{aligned}
$$

where $T_{0 ; 3}^{0}$ is again the result of [27].

\section{Seiberg-Witten Curve}

\subsection{Periods of rational elliptic surfaces}

Local mirror of the IIA string on $K_{B_{9}}$ with the Kähler moduli (2.5) is the IIB string on the degenerate Calabi-Yau threefold given by the two equations in $(\bar{x}, \bar{y}, \tilde{x}, \tilde{y}, u)[27]$ :

$$
\begin{aligned}
(\bar{y})^{2} & =4(\bar{x})^{3}-f(u ; \tau, \mu) \bar{x}-g(u ; \tau, \mu), \\
\tilde{x} \tilde{y} & =u-u^{*}
\end{aligned}
$$

where the first equation (8.1) itself describes the family of rational elliptic surfaces $S_{9}$ in Weierstrass form, the nine moduli $(\tau, \mu)$ of which should be encoded as

$$
\begin{array}{ll}
f(u ; \tau, \mu)=\sum_{i=0}^{4} f_{4-i}(\tau, \mu) u^{i}, & f_{0}(\tau)=\frac{4}{3} \pi^{4} E_{4}(\tau), \\
g(u ; \tau, \mu)=\sum_{i=0}^{6} g_{6-i}(\tau, \mu) u^{i}, & g_{0}(\tau)=\frac{8}{27} \pi^{6} E_{6}(\tau) .
\end{array}
$$

The determination of the precise forms of $f$ and $g$ for given moduli $(\tau, \mu)$ will be discussed in section 9. The base of the elliptic fibration $\pi: S_{9} \mapsto \mathbf{P}^{1}$ is the $u$-plane and we have a rational two-form $\Omega=\mathrm{d} \bar{x} / \bar{y} \wedge \mathrm{d} u$ on it inherited from the holomorphic three-form $\Omega \wedge \mathrm{d} \tilde{x} / \tilde{x}$ on the Calabi-Yau threefold through the Poincaré residue. Note that the pair $S_{9}$ given by (8.1) and $\Omega$ is nothing but the ingredients of the Seiberg-Witten curve [38] 
that describes $4 \mathrm{D}$ E-string $[10,12]$. There exists a $\mathbf{C}^{*}$-action on $S_{9}$ which preserves the two-form $\Omega[10,12]$ :

$$
(\bar{x}, \bar{y}, u) \mapsto\left(\lambda^{2} \bar{x}, \lambda^{3} \bar{y}, \lambda u\right), \quad \lambda \in \mathbf{C}^{*}
$$

Let $E_{u}:=\pi^{-1}(u)$ be the fiber at $u$; the leading terms $f_{0}$ and $g_{0}$ are fixed by the physical requirement that $E_{\infty}$, the fiber at infinity, has the modulus $\tau$.

Let us introduce the coordinates at $u=\infty$ by $(x, y, t)=\left(\bar{x} u^{2}, \bar{y} u^{3}, 1 / u\right)$, in terms of which the defining equation of $E_{\infty}$ can be written in a canonical form:

$$
E_{\infty}: y^{2}=4 x^{3}-f_{0}(\tau) x-g_{0}(\tau)
$$

There exits a pair of one-cycles on $E_{\infty}(\alpha, \beta)$ such that

$$
\int_{\alpha} \frac{\mathrm{d} x}{y}=1, \quad \int_{\beta} \frac{\mathrm{d} x}{y}=\tau .
$$

The rational two-form $\Omega$ now takes the form $\Omega=\mathrm{d} t / t \wedge \mathrm{d} x / y$, from which we see that the Poincaé residue of $\Omega$ along $E_{\infty}$ is nothing but the canonical one-form on $E_{\infty}$ :

$$
\operatorname{Res}_{E_{\infty}}(\Omega)=\frac{\mathrm{d} x}{y}
$$

We can identify $E_{\infty}$ with the complex torus $\mathbf{C} /(\mathbf{Z} \tau+\mathbf{Z})$ through the uniformization $(x, y)=\left(\wp(\tau \mid \nu), \wp^{\prime}(\tau \mid \nu)\right)$, where $\nu$ is the coordinate of the covering space $\mathbf{C}$ of the torus and the Weierstrass $\wp$ function is defined by

$$
\wp(\tau \mid \nu)=\frac{1}{\nu^{2}}+\sum_{\omega \in \mathbf{Z} \tau+\mathbf{Z}}^{\prime}\left[\frac{1}{(\nu-\omega)^{2}}-\frac{1}{\omega^{2}}\right] .
$$

Note that $\mathrm{d} x / y$ is pulled-back by this isomorphism to $\mathrm{d} \nu$, which makes (8.6) rather trivial. The point $\nu \bmod \mathbf{Z} \tau+\mathbf{Z}$ of the torus will be frequently used to refer to the point $\left(\wp(\tau \mid \nu), \wp^{\prime}(\tau \mid \nu)\right)$ of $E_{\infty}$ below.

We also introduce the homogeneous coordinates $\left(x_{0}, x_{1}, x_{2}\right)$ of $\mathbf{P}^{2}$ with $x=x_{1} / x_{0}$, $y=x_{2} / x_{0}$, so that we can realize $E_{\infty}$ as a plane curve defined by the ternary cubic $P$ :

$$
P\left(x_{0}, x_{1}, x_{2}\right):=x_{0} x_{2}^{2}-4 x_{1}^{3}+f_{0}(\tau) x_{0}^{2} x_{1}+g_{0}(\tau) x_{0}^{3} .
$$

The twelve normalized periods $\left(1, \tau, \sigma, \mu, \partial_{\sigma} F_{0}\right)$ of the E-string can be obtained as the periods of the Seiberg-Witten curve (8.1) [10, 12, 24, 29, 27, 14]. 
To see this, let $(\alpha(u), \beta(u))$ be the standard symplectic basis of the fiber $H_{1}\left(E_{u}\right)$ which extends the one $(\alpha, \beta)$ at $u=\infty$. It is clear from (8.6) and (8.7) that the two periods 1 , $\tau$ can be recovered by the integrals [14]:

$$
1=-\frac{1}{2 \pi \mathrm{i}} \oint_{u=\infty} \mathrm{d} u \oint_{\alpha(u)} \frac{\mathrm{d} \bar{x}}{\bar{y}}, \quad \tau=-\frac{1}{2 \pi \mathrm{i}} \oint_{u=\infty} \mathrm{d} u \oint_{\beta(u)} \frac{\mathrm{d} \bar{x}}{\bar{y}} .
$$

Evaluation of the Wilson lines $\mu=\sum_{i=1}^{8} \mu_{i} \omega_{i}$ needs a more careful study of periods, since it deals with a seemingly divergent integral due to the pole of $\Omega$.

Notice first that $\Omega$ is a holomorphic two-form on $S_{9}-E_{\infty}$. Then we are naturally lead to define the period map $\hat{\varrho}[26]$ of $\Omega$ by

$$
\hat{\varrho}: H_{2}\left(S_{9}-E_{\infty}\right) \longrightarrow \mathbf{C}, \quad \hat{\chi}(D):=\frac{1}{2 \pi \mathrm{i}} \int_{D} \Omega .
$$

The non-trivial part of the homology exact sequence of the pair $\left(S_{9}, S_{9}-E_{\infty}\right)$ :

$$
\cdots \longrightarrow H_{i}\left(S_{9}-E_{\infty}\right) \longrightarrow H_{i}\left(S_{9}\right) \longrightarrow H_{i}\left(S_{9}, S_{9}-E_{\infty}\right) \longrightarrow H_{i-1}\left(S_{9}-E_{\infty}\right) \longrightarrow \cdots,
$$

can be written as

$$
0 \rightarrow H_{1}\left(E_{\infty}\right) \stackrel{\partial_{*}}{\longrightarrow} H_{2}\left(S_{9}-E_{\infty}\right) \stackrel{i_{*}}{\longrightarrow} H_{2}\left(S_{9}\right) \stackrel{j_{*}}{\longrightarrow} H_{0}\left(E_{\infty}\right) \rightarrow 0
$$

where we used the Poincaré duality $H_{i}\left(S_{9}, S_{9}-E_{\infty}\right) \cong H^{4-i}\left(E_{\infty}\right)$ and $H_{1}\left(S_{9}-E_{\infty}\right) \cong 0$.

There are two points: first, $\partial_{*} \alpha$ and $\partial_{*} \beta$ are just the elements of $H_{2}\left(S_{9}-E_{\infty}\right)$ appeared in (8.9), from which it follows immediately that $\varrho\left(\partial_{*} \alpha\right)=1$, and $\varrho\left(\partial_{*} \beta\right)=\tau$, that is, $\varrho \cdot \partial_{*}$ is nothing but the period map of $E_{\infty}$ by $\mathrm{d} x / y$; second, $j_{*}: H_{2}\left(S_{9}\right) \rightarrow H_{0}\left(E_{\infty}\right) \cong \mathbf{Z}$ simply counts the intersection number of a divisor with $E_{\infty}$. As the homology class of $E_{\infty}$ is $[\delta]$, we see that $\operatorname{Ker} j_{*}=\operatorname{Im} i_{*}$ can be identified with $L\left(\boldsymbol{E}_{8}^{(1)}\right)$.

We conclude that $\varrho$ induces the homomorphism of additive groups:

$$
\varrho: L\left(\boldsymbol{E}_{8}^{(1)}\right) \rightarrow \mathbf{C} \bmod \mathbf{Z} \tau+\mathbf{Z}
$$

In other words, we can regularize systematically the integral of the rational form $\Omega$ over $L\left(\boldsymbol{E}_{8}^{(1)}\right)$ at the expense of the additive ambiguity $\mathbf{Z} \tau+\mathbf{Z}$.

It is possible to describe $\chi$ quite explicitly in terms of the moduli of $S_{9}$. Let $p_{i}:=$ $\left(\wp\left(\tau \mid \nu_{i}\right), \wp^{\prime}\left(\tau \mid \nu_{i}\right)\right)$ be the intersection point in $S_{9}$ of $E_{\infty}$ with the $i$ th exceptional divisor 
$\mathcal{E}_{i}$. Then the rational elliptic surface $S_{9}$ is obtained by blow-up of $\mathbf{P}^{2}$ at these nine points, which also satisfy

$$
\sum_{i=1}^{9} \nu_{i}=0 \bmod \mathbf{Z} \tau+\mathbf{Z}
$$

because they are the intersection points of two cubics in $\mathbf{P}^{2}$, that is, there exists another cubic $Q$ such that $\left\{\nu_{i}\right\}=\{P=0\} \cap\{Q=0\}$. The rational elliptic surface $S_{9}$ in question is then expressed as the hypersurface $P+t Q=0$ in $\mathbf{P}^{2} \times \mathbf{P}^{1}$; this is the same as the realization of the $\boldsymbol{E}_{0}$ model, but it is the complex structure that matters here.

We can show that $\varrho$ is given by $[26,34]$

$$
\begin{aligned}
\varrho\left(\mathcal{E}_{i}-\mathcal{E}_{j}\right) & =\nu_{i}-\nu_{j}, \\
\varrho\left(l-\mathcal{E}_{i}-\mathcal{E}_{j}-\mathcal{E}_{k}\right) & =-\nu_{i}-\nu_{j}-\nu_{k},
\end{aligned}
$$

where $\nu_{i}$ s are defined only up to addition of $\mathbf{Z} \tau+\mathbf{Z}$.

Let us see how (8.13) is obtained. Choose a path $\gamma_{i, j}$ which connects $p_{j}$ and $p_{i}$ on $E_{\infty}$ and let $T_{i, j}$ be a closed tubular neighbourhood of $\gamma_{i, j}$ in $S_{9}-E_{\infty}$, such that each of $\mathcal{E}_{i} \cap T_{i, j}=D_{i}$ and $\mathcal{E}_{j} \cap T_{i, j}=D_{j}$ is its fiber. Then $\left(\mathcal{E}_{i}-D_{i}\right) \cup \partial T_{i, j} \cup\left(\mathcal{E}_{j}-D_{j}\right)$ becomes an oriented topological manifold homologous to $\mathcal{E}_{i}-\mathcal{E}_{j}$ and disjoint from $E_{\infty}$. In physical terms, $\partial T_{i, j}$ is a "wormhole" connecting the two "universes" $\mathcal{E}_{i}$ and $\mathcal{E}_{j}$, with the singular points $p_{i}, p_{j}$ replaced by a "black and white hole". Now the evaluation of the left hand side of (8.13) proceeds as follows:

$$
\frac{1}{2 \pi \mathrm{i}} \int_{\mathcal{E}_{i}-\mathcal{E}_{j}} \Omega=\frac{1}{2 \pi \mathrm{i}} \int_{\partial T_{i, j}} \Omega=\int_{\gamma_{i, j}} \operatorname{Res}_{E_{\infty}} \Omega=\int_{p_{j}}^{p_{i}} \frac{\mathrm{d} x}{y},
$$

which yields precisely the right hand side of (8.13), the ambiguity of which comes from the choice of the path $\gamma_{i, j}$.

As for $(8.14)$, we first combine the divisor in question as $\left(l-\mathcal{E}_{i}-\mathcal{E}_{j}\right)-\mathcal{E}_{k}$. We can then take as $l$ the transform of the line on $\mathbf{P}^{2}$ that passes through the two points $\left\{p_{i}, p_{j}\right\}$. Now we see that $\left(l-\mathcal{E}_{i}-\mathcal{E}_{j}\right)$ is an effective curve intersecting with $E_{\infty}$ at $-p_{i}-p_{j}$, the coordinates of which are $\left(\wp(\tau \mid \nu), \wp^{\prime}(\tau \mid \nu)\right)$, with $\nu:=-\nu_{i}-\nu_{j}$. At this point, the problem 
is reduced to (8.13), so that (8.14) follows. We note that as a consequence of (8.14),

$$
\begin{aligned}
\varrho([\delta]) & =\varrho\left(l-\mathcal{E}_{1}-\mathcal{E}_{2}-\mathcal{E}_{3}\right)+\varrho\left(l-\mathcal{E}_{4}-\mathcal{E}_{5}-\mathcal{E}_{6}\right)+\varrho\left(l-\mathcal{E}_{7}-\mathcal{E}_{8}-\mathcal{E}_{9}\right) \\
& =-\sum_{i=1}^{9} \nu_{i}=0 \bmod \mathbf{Z} \tau+\mathbf{Z}
\end{aligned}
$$

which is consistent with the fact that we can take a generic fiber $E_{u}$ as a representative of $[\delta]$, thus $\left.\Omega\right|_{E_{u}}$ vanishes identically, since $E_{u}$ is a holomorphic curve disjoint from $E_{\infty}$.

We do not have much to say about the Seiberg-Witten periods [38], $\sigma$ and $\partial_{\sigma} F_{0}$. These are not periods of $S_{9}$ in the sense above, and roughly given by

$$
\sigma=-\int \mathrm{d} u \oint_{\alpha(u)} \frac{\mathrm{d} \bar{x}}{\bar{y}}, \quad \frac{1}{(2 \pi \mathrm{i})^{2}}\left(\frac{\partial F_{0}}{\partial \sigma}\right)=\int \mathrm{d} u\left(\oint_{\beta(u)} \frac{\mathrm{d} \bar{x}}{\bar{y}}-\tau \int_{\alpha(u)} \frac{\mathrm{d} \bar{x}}{\bar{y}}\right),
$$

a detailed account of which we have already given for the six two-parameter models in (4.65), (4.66) and (4.67) for $\sigma$ and in (4.69), (4.70), and (4.71) for $\partial_{\sigma} F_{0}$, with the correspondence of the bare parameters $z_{1} \propto u^{-1}$ in mind. Explicit evaluation and instanton expansion of these Seiberg-Witten integrals in terms of modular forms has been done for the $\boldsymbol{E}_{8}$ model in [29].

\subsection{Wilson lines}

Based on the results in the last subsection, we find the Wilson lines $[38,10,12,27]$ to be

$$
\mu_{i}=\frac{1}{2 \pi \mathrm{i}} \varrho\left(\left[\alpha_{i}\right]\right)= \begin{cases}\nu_{i}-\nu_{i+1}, & i=1, \ldots, 7 \\ -\nu_{1}-\nu_{2}-\nu_{3}, & i=8\end{cases}
$$

where $\left[\alpha_{i}\right] \in H_{2}\left(S_{9}\right)$ is defined in $(2.3)$.

The Euclidean coordinates (2.6) of the Wilson lines $\left(m_{i}\right)$ and the base points of the 
cubic pencil $\nu_{i}=E_{\infty} \cap \mathcal{E}_{i}$ are related to each other by

$$
\begin{aligned}
& \left(\begin{array}{l}
m_{1} \\
m_{2} \\
m_{3} \\
m_{4} \\
m_{5} \\
m_{7} \\
m_{8}
\end{array}\right)=-\frac{1}{2}\left(\begin{array}{llllllll}
1 & 2 & 0 & 0 & 0 & 0 & 0 & 0 \\
1 & 0 & 2 & 0 & 0 & 0 & 0 & 0 \\
1 & 0 & 0 & 2 & 0 & 0 & 0 & 0 \\
1 & 0 & 0 & 0 & 2 & 0 & 0 & 0 \\
1 & 0 & 0 & 0 & 0 & 2 & 0 & 0 \\
1 & 0 & 0 & 0 & 0 & 0 & 2 & 0 \\
1 & 0 & 0 & 0 & 0 & 0 & 0 & 2 \\
1 & 2 & 2 & 2 & 2 & 2 & 2 & 2
\end{array}\right)\left(\begin{array}{l}
\nu_{1} \\
\nu_{2} \\
\nu_{3} \\
\nu_{4} \\
\nu_{5} \\
\nu_{6} \\
\nu_{7} \\
\nu_{8}
\end{array}\right) \\
& \left(\begin{array}{l}
\nu_{1} \\
\nu_{2} \\
\nu_{3} \\
\nu_{4} \\
\nu_{5} \\
\nu_{6} \\
\nu_{7} \\
\nu_{8}
\end{array}\right)=\frac{1}{6}\left(\begin{array}{rrrrrrrr}
-2 & -2 & -2 & -2 & -2 & -2 & -2 & 2 \\
-5 & 1 & 1 & 1 & 1 & 1 & 1 & -1 \\
1 & -5 & 1 & 1 & 1 & 1 & 1 & -1 \\
1 & 1 & -5 & 1 & 1 & 1 & 1 & -1 \\
1 & 1 & 1 & -5 & 1 & 1 & 1 & -1 \\
1 & 1 & 1 & 1 & -5 & 1 & 1 & -1 \\
1 & 1 & 1 & 1 & 1 & -5 & 1 & -1 \\
1 & 1 & 1 & 1 & 1 & 1 & -5 & -1
\end{array}\right)\left(\begin{array}{l}
m_{1} \\
m_{2} \\
m_{3} \\
m_{4} \\
m_{5} \\
m_{7} \\
m_{8}
\end{array}\right) .
\end{aligned}
$$

\section{Inverse Problem}

\subsection{General strategy}

We are interested in the following problem: given the eight Wilson lines $\mu=\sum_{i=1}^{8} \mu_{i} \omega_{i}$, or equivalently the nine points $\left(\nu_{i}\right)_{i=1}^{9}$ on the torus $\mathbf{C} /(\mathbf{Z} \tau+\mathbf{Z})$, which satisfy (8.12), find the corresponding Seiberg-Witten curve (8.1). In other words, we want to know explicitly the two functions $f(u ; \tau, \mu)$ in $(8.2)$ and $g(u ; \tau, \mu)$ in $(8.3)$ as functions of the moduli.

We will solve this problem in two steps [12]: in the first step, we obtain the cubic pencil $P+t Q$ in $\mathbf{P}^{2}$ for the given base points $\left(\nu_{i}\right)$, which is achieved by consideration based on the elliptic function theory; and in the second step, we transform the cubic pencil into the Weierstrass form (8.1), with the help of the classical theory of algebraic invariants.

First step We claim that the curve defined by the cubic $Q\left(x_{0}, x_{1}, x_{2}\right)$ shown below passes through the nine points $\left\{\left(1, \wp\left(\tau \mid \nu_{i}\right), \wp^{\prime}\left(\tau \mid \nu_{i}\right)\right)\right\}$, where $\left(\nu_{i}\right)$ are taken to be generic 
except (8.12):

$$
Q=\left|\begin{array}{ccccccccc}
x_{0}^{3} & x_{0}^{2} x_{1} & x_{0}^{2} x_{2} & x_{0} x_{1}^{2} & x_{0} x_{1} x_{2} & x_{0} x_{2}^{2} & x_{1}^{2} x_{2} & x_{1} x_{2}^{2} & x_{2}^{3} \\
1 & \wp_{1} & \wp_{1}^{\prime} & \wp_{1}^{2} & \wp_{1} \wp_{1}^{\prime} & \left(\wp_{1}^{\prime}\right)^{2} & \wp_{1}^{2} \wp_{1}^{\prime} & \wp_{1}\left(\wp_{1}^{\prime}\right)^{2} & \left(\wp_{1}^{\prime}\right)^{3} \\
1 & \wp_{2} & \wp_{2}^{\prime} & \wp_{2}^{2} & \wp_{2} \wp_{2}^{\prime} & \left(\wp_{2}^{\prime}\right)^{2} & \wp_{2}^{2} \wp_{2}^{\prime} & \wp_{2}\left(\wp_{2}^{\prime}\right)^{2} & \left(\wp_{2}^{\prime}\right)^{3} \\
1 & \wp_{3} & \wp_{3}^{\prime} & \wp_{3}^{2} & \wp_{3} \wp_{3}^{\prime} & \left(\wp_{3}^{\prime}\right)^{2} & \wp_{3}^{2} \wp_{3}^{\prime} & \wp_{3}\left(\wp_{3}^{\prime}\right)^{2} & \left(\wp_{3}^{\prime}\right)^{3} \\
1 & \wp_{4} & \wp_{4}^{\prime} & \wp_{4}^{2} & \wp_{4} \wp_{4}^{\prime} & \left(\wp_{4}^{\prime}\right)^{2} & \wp_{4}^{2} \wp_{4}^{\prime} & \wp_{4}\left(\wp_{4}^{\prime}\right)^{2} & \left(\wp_{4}^{\prime}\right)^{3} \\
1 & \wp_{5} & \wp_{5}^{\prime} & \wp_{5}^{2} & \wp_{5} \wp_{5}^{\prime} & \left(\wp_{5}^{\prime}\right)^{2} & \wp_{5}^{2} \wp_{5}^{\prime} & \wp_{5}\left(\wp_{5}^{\prime}\right)^{2} & \left(\wp_{5}^{\prime}\right)^{3} \\
1 & \wp_{6} & \wp_{6}^{\prime} & \wp_{6}^{2} & \wp_{6} \wp_{6}^{\prime} & \left(\wp_{6}^{\prime}\right)^{2} & \wp_{6}^{2} \wp_{6}^{\prime} & \wp_{6}\left(\wp_{6}^{\prime}\right)^{2} & \left(\wp_{6}^{\prime}\right)^{3} \\
1 & \wp_{7} & \wp_{7}^{\prime} & \wp_{7}^{2} & \wp_{7} \wp_{7}^{\prime} & \left(\wp_{7}^{\prime}\right)^{2} & \wp_{7}^{2} \wp_{7}^{\prime} & \wp_{7}\left(\wp_{7}^{\prime}\right)^{2} & \left(\wp_{7}^{\prime}\right)^{3} \\
1 & \wp_{8} & \wp_{8}^{\prime} & \wp_{8}^{2} & \wp_{8} \wp_{8}^{\prime} & \left(\wp_{8}^{\prime}\right)^{2} & \wp_{8}^{2} \wp_{8}^{\prime} & \wp_{8}\left(\wp_{8}^{\prime}\right)^{2} & \left(\wp_{8}^{\prime}\right)^{3}
\end{array}\right|,
$$

where we have used the abbreviated notation $\wp_{i}=\wp\left(\tau \mid \nu_{i}\right), \wp_{i}^{\prime}=\wp^{\prime}\left(\tau \mid \nu_{i}\right)$.

The proof is quite simple if we recall some fundamental theorems of the elliptic function theory [20]; first $h(\tau \mid \nu):=Q\left(1, \wp(\tau \mid \nu), \wp^{\prime}(\tau \mid \nu)\right)$ is an elliptic function with the only pole of ninth order at $\nu=0$; then as $h(\tau \mid \nu)$ has eight simple zeros at $\nu=\nu_{1}, \ldots, \nu_{8}$ by construction, the ninth zero should be $\nu=-\sum_{i=1}^{8} \nu_{i}=\nu_{9}$.

In fact, $h(\tau \mid \nu)$ admits the following concise expression [40, III, p.98-99]:

$$
h\left(\tau \mid \nu_{0}\right)=2^{10} \frac{\sigma\left(\tau \mid \sum_{i=0}^{8} \nu_{i}\right) \prod_{i<j} \sigma\left(\tau \mid \nu_{j}-\nu_{i}\right)}{\prod_{i=0}^{8} \sigma\left(\tau \mid \nu_{i}\right)^{9}},
$$

where $\sigma(\tau \mid \nu)$ is the Weierstrass sigma function

$$
\sigma(\tau \mid \nu):=\nu \prod_{\omega \in \mathbf{Z} \tau+\mathbf{Z}}^{\prime}\left(1-\frac{\nu}{\omega}\right) \mathrm{e}^{\left(\frac{\nu}{\omega}\right)+\frac{1}{2}\left(\frac{\nu}{\omega}\right)^{2}}=\frac{1}{2 \pi} \mathrm{e}^{\frac{1}{6}(\pi \nu)^{2} E_{2}(\tau)} \frac{\vartheta_{1}(\tau \mid \nu)}{\eta(\tau)^{3}} .
$$

The $E_{2}(\tau)$ factors in the sigma functions cancel out in (9.2). Curiously, we encounter the same function $\vartheta_{1}(\tau \mid \nu) / \eta(\tau)^{3}$ as in (2.16).

The cubic curve $Q=0$ intersects with $E_{\infty}$ at the nine points $\left\{\left(1, \wp_{i}, \wp_{i}^{\prime}\right)\right\}$. Note that $Q$ never coincides with the original cubic $P$ since $Q$ lacks the $x_{1}^{3}$ term [12].

Therefore we have found for the given moduli parameters $(\tau, \mu)$ the rational elliptic surface $S_{9}$ in the form of a cubic pencil $P+t Q$, where $P$ is given in (8.8) and $Q$ in (9.1).

If $\left(\nu_{i}\right)$ are not generic, e.g., if $\nu_{i}=\nu_{j}$, then the right hand side of (9.1) vanishes identically. However an appropriate limiting procedure such as $\nu_{j} \rightarrow \nu_{i}$ should still produce a non-trivial solution. In fact, in the following subsection we treat several models with degenerate Wilson line parameters, where $Q$ factorizes into lower degree polynomials. 
Second step What is really in need is the Seiberg-Witten form (8.1) of $S_{9}$, which is given by the cubic pencil at this point. In principle, we can find a coordinate transformation of $\left(x_{0}, x_{1}, x_{2}\right)$ by $G L(3 ; \mathbf{C})$ which takes the cubic pencil $P+t Q$ above into the Weierstrass form [12], but it seems very difficult to perform this task in general. We can in fact skip this difficulty to reach the Weierstrass form (8.1) directly.

To see this, first recall that there exists a natural action of $G L(3 ; \mathbf{C})$ on the space of the ternary cubic forms, a general member $R$ of which we write as

$$
R:=\sum_{p+q+r=3}\left(\frac{3 !}{p ! q ! r !}\right) a_{p q r} x_{0}^{p} x_{1}^{q} x_{2}^{r} .
$$

It is well-known that the ring of the projective invariants are the polynomial ring over $\mathbf{C}$ generated by the two basic invariants $\mathcal{S}$ and $\mathcal{T}$, that is, $\left(\mathbf{C}\left[a_{p q r}\right]\right)^{P G L(3 ; \mathbf{C})}=\mathbf{C}[\mathcal{S}, \mathcal{T}]$ which can be obtained by the formula [15, II.7]:

$$
\begin{aligned}
\operatorname{Hess}\left\{\alpha R+\frac{\beta}{36} \operatorname{Hess}(R)\right\} & =\left(432 \alpha^{2} \beta \mathcal{S}+1728 \beta^{3} \mathcal{S}^{2}-216 \alpha \beta^{2} \mathcal{T}\right) R \\
& +\left(\alpha^{3}+2 \beta^{3} \mathcal{T}-12 \alpha \beta^{2} \mathcal{S}\right) \operatorname{Hess}(R)
\end{aligned}
$$

where $\operatorname{Hess}(R):=\left|\left(\partial_{i} \partial_{j} R\right)\right|$ is the Hessian of $R$, which is another cubic. We give in (A.1) and (A.2) the explicit forms of these two invariants, which indeed coincide with those in [39, Prop.4.4.7] and [39, Exm.4.5.3].

Any generic cubic $R$ can then be transformed by $G L(3 ; \mathbf{C})$ to the Weierstrass form:

$$
R \mapsto x_{2}^{2} x_{0}-4 x_{1}^{3}+f x_{1} x_{0}^{2}+g x_{0}^{3}, \quad f=\frac{27}{4} \mathcal{S}, g=\frac{27}{64} \mathcal{T}
$$

This technique enables us to find the Seiberg-Witten form (8.1) of the rational elliptic surface $S_{9}$ given by a cubic pencil $P+t Q$.

Fixed Wilson lines In the remaining of this subsection, we take two models with fixed Wilson lines as a warming-up exercise.

Let us first take the $\boldsymbol{E}_{8}$ model the Wilson lines of which are $\nu_{i}=0$ for all $i$. Thus we need a cubic $Q\left(x_{0}, x_{1}, x_{2}\right)$ which intersects with $E_{\infty}$ nine times at $\left(x_{0}, x_{1}, x_{2}\right)=(0,1,0)$ corresponding to $\nu=0$. The triple line $Q=x_{0}^{3}$ does the job, simply because $\nu=0$ is one of the inflection points of $E_{\infty}$. 
The resulting cubic pencil $P+t Q$ can easily be converted to the Weierstrass form in the original variables $(\bar{x}, \bar{y})$ :

$$
(\bar{y})^{2}=4(\bar{x})^{3}-f_{0}(\tau) u^{4} \bar{x}-\left(g_{0}(\tau) u^{6}+u^{5}\right),
$$

which is the Seiberg-Witten curve of the $\boldsymbol{E}_{8}$ model found in [12].

Next consider the model with the nine inflection points $\left\{\frac{1}{3}(a \tau+b) \mid a, b=0,1,2\right\}$ as the base points of the cubic pencil. Note that these nine points sum up to zero.

We can find easily also in this case the cubic $Q$; it is simply the Hessian of $P$ :

$$
\operatorname{Hess}(P)=-8\left(f_{0}(\tau)^{2} x_{0}^{3}+36 g_{0}(\tau) x_{0}^{2} x_{1}+12 f_{0}(\tau) x_{0} x_{1}^{2}-12 x_{1} x_{2}^{2}\right) .
$$

This follows from the fact that the four $x$ coordinates $\left\{\wp\left(\tau \mid \frac{1}{3}(a \tau+b)\right) \mid(a, b) \neq(0,0)\right\}$ are the roots of the equation: $4 x^{4}-2 f_{0}(\tau) x^{2}-4 g_{0}(\tau) x-f_{0}(\tau)^{2} / 12=0$.

The calculation of the basic algebraic invariants $\mathcal{S}, \mathcal{T}$ of the cubic $P+t \operatorname{Hess}(P)$ yields the Weierstrass form (8.1) of the cubic pencil with

$$
\begin{aligned}
f(u ; \tau)= & \frac{4}{3} \pi^{4}\left(E_{4}(\tau) u^{4}-4 E_{6}(\tau) u^{3}+6 E_{4}(\tau)^{2} u^{2}-4 E_{4} E_{6}(\tau) u+4 E_{6}(\tau)^{2}-3 E_{4}(\tau)^{3}\right), \\
g(u ; \tau)= & \frac{8}{27} \pi^{6}\left(E_{6}(\tau) u^{6}-6 E_{4}(\tau)^{2} u^{5}+15 E_{4} E_{6}(\tau) u^{4}-20 E_{6}(\tau)^{2} u^{3}+15 E_{4}^{2} E_{6}(\tau) u^{2}\right. \\
& \left.+6 E_{4}\left(2 E_{6}^{2}-3 E_{4}^{3}\right)(\tau) u+E_{6}\left(9 E_{4}^{3}-8 E_{6}^{2}\right)(\tau)\right) .
\end{aligned}
$$

Recall here that $f_{0}(\tau)=4 / 3 \pi^{4} E_{4}(\tau), g_{0}(\tau)=8 / 27 \pi^{6} E_{6}(\tau)$.

We can see that the partition function of the singly-winding sector $Z_{g, 1}(\tau)$ vanishes identically for this model due to the theta function formula [40, II, p.148], the physical meaning of which is yet to be clarified.

\subsection{Several models with a few Wilson lines}

Two Wilson lines The model with two Wilson lines, that is, $\mu=m_{1} \mathbf{e}_{1}+m_{2} \mathbf{e}_{2}$ has been investigated in [27], where the Seiberg-Witten curve were found and the partition function $Z_{0,1}\left(\tau \mid m_{i}\right)$ has been derived directly from it.

We find the nine base points of the cubic pencil representing the rational elliptic surface to be $\left\{\nu_{i}\right\}=\left\{0,0,0,0,0, m_{+},-m_{+}, m_{-},-m_{-}\right\}$, where $m_{ \pm}=\left(m_{1} \pm m_{2}\right) / 2$. The cubic $Q$ which passes through these nine points can easily be identified. In fact, the nine 
points are decomposed into the three triples $\{0,0,0\},\left\{0, m_{+},-m_{+}\right\},\left\{0, m_{-},-m_{-}\right\}$, each of which defines a line in $\mathbf{P}^{2}$ :

$$
L_{0}=x_{0}, \quad L_{+}=x_{1}-\wp\left(\tau \mid m_{+}\right) x_{0}, \quad L_{-}=x_{1}-\wp\left(\tau \mid m_{-}\right) x_{0} .
$$

Thus the cubic $Q$ is found to be $Q=x_{0}\left(x_{1}-\wp\left(\tau \mid m_{+}\right) x_{0}\right)\left(x_{1}-\wp\left(\tau \mid m_{-}\right) x_{0}\right)$.

Now calculation of the basic algebraic invariants of the ternary cubic $P+t Q$ tells us its Weierstrass form (8.1):

$$
\begin{aligned}
& f\left(u ; \tau, m_{ \pm}\right)=f_{0} u^{4}-\left(\wp_{+}+\wp_{-}\right) u^{3}+\frac{1}{12} u^{2}, \\
& g\left(u ; \tau, m_{ \pm}\right)=g_{0} u^{6}+\left(\wp_{+} \wp_{-}+\frac{f_{0}}{12}\right) u^{5}-\frac{1}{12}\left(\wp_{+}+\wp_{-}\right) u^{4}+\frac{1}{216} u^{3},
\end{aligned}
$$

where $\wp_{ \pm}=\wp\left(\tau \mid m_{ \pm}\right)$. Indeed, using the formula

$$
\frac{1}{\pi^{2}} \wp(\tau \mid m)=-\frac{1}{3}\left(\vartheta_{3}(\tau)^{4}+\vartheta_{2}(\tau)^{4}\right)+\vartheta_{3}(\tau)^{2} \vartheta_{2}(\tau)^{2}\left(\frac{\vartheta_{4}(\tau \mid m)}{\vartheta_{1}(\tau \mid m)}\right)^{2},
$$

and a rescaling (8.4), it can be shown that the above form coincides with the one in $[27$, (A.1)], which was obtained through a reasoning quite different from the one here, modulo some misprints.

Three Wilson lines Consider the model with the Wilson lines $\mu=\sum_{i=1}^{3} m_{i} \mathbf{e}_{i}$, where $m_{i}$ are chosen to be generic. A $W\left(\boldsymbol{E}_{8}\right)$ action simplifies the nine base points of the cubic pencil to be $\left\{\nu_{i}\right\}=\left\{0,0,0,0,0, \zeta_{0}, \zeta_{1}, \zeta_{2}, \zeta_{3}\right\}$, where

$$
\left(\zeta_{0}, \zeta_{1}, \zeta_{2}, \zeta_{3}\right)=\frac{1}{2}\left(m_{1}, m_{2}, m_{3}\right)\left(\begin{array}{rrrr}
-1 & -1 & 1 & 1 \\
-1 & 1 & -1 & 1 \\
-1 & 1 & 1 & -1
\end{array}\right)
$$

We see immediately that $\{0,0,0\}$ determines the line $x_{0}=0$ and the other six points $\left\{0,0, \zeta_{0}, \zeta_{1}, \zeta_{2}, \zeta_{3}\right\}$ the conic of the form $C=a_{0} x_{0}^{2}+a_{1} x_{0} x_{1}+a_{2} x_{0} x_{2}+a_{3} x_{1}^{2}$, the precise coefficients of which are determined by the formula

$$
\begin{gathered}
C\left(x_{0}, x_{1}, x_{2}\right)=\left|\begin{array}{cccc}
x_{0}^{2} & x_{0} x_{1} & x_{0} x_{2} & x_{1}^{2} \\
1 & \wp_{1} & \wp_{1}^{\prime} & \wp_{1}^{2} \\
1 & \wp_{2} & \wp_{2}^{\prime} & \wp_{2}^{2} \\
1 & \wp_{3} & \wp_{3}^{\prime} & \wp_{3}^{2}
\end{array}\right|, \\
a_{0}=\left|\begin{array}{lll}
\wp_{1} & \wp_{1}^{\prime} & \wp_{1}^{2} \\
\wp_{2} & \wp_{2}^{\prime} & \wp_{2}^{2} \\
\wp_{3} & \wp_{3}^{\prime} & \wp_{3}^{2}
\end{array}\right|, a_{1}=-\left|\begin{array}{ccc}
1 & \wp_{1}^{\prime} & \wp_{1}^{2} \\
1 & \wp_{2}^{\prime} & \wp_{2}^{2} \\
1 & \wp_{3}^{\prime} & \wp_{3}^{2}
\end{array}\right|, a_{2}=\left|\begin{array}{ccc}
1 & \wp_{1} & \wp_{1}^{2} \\
1 & \wp_{2} & \wp_{2}^{2} \\
1 & \wp_{3} & \wp_{3}^{2}
\end{array}\right|, a_{3}=-\left|\begin{array}{lll}
1 & \wp_{1} & \wp_{1}^{\prime} \\
1 & \wp_{2} & \wp_{2}^{\prime} \\
1 & \wp_{3} & \wp_{3}^{\prime}
\end{array}\right|,
\end{gathered}
$$


where $\wp_{i}=\wp\left(\tau \mid \zeta_{i}\right), \wp_{i}^{\prime}=\wp^{\prime}\left(\tau \mid \zeta_{i}\right)$.

The cubic passing through the nine points are determined to be

$$
Q=x_{0}\left(a_{0} x_{0}^{2}+a_{1} x_{0} x_{1}+a_{2} x_{0} x_{2}+a_{3} x_{1}^{2}\right)
$$

and the Seiberg-Witten form (8.1) of it is written in terms of $\left(a_{0}, a_{1}, a_{2}, a_{3}\right)$ as

$$
\begin{aligned}
& f\left(u ; \tau, m_{i}\right)=f_{0} u^{4}+a_{1} u^{3}+\frac{1}{12} a_{3}^{2} u^{2}, \\
& g\left(u ; \tau, m_{i}\right)=g_{0} u^{6}+\left(a_{0}+\frac{f_{0}}{12} a_{3}\right) u^{5}+\frac{1}{12}\left(a_{1} a_{3}-3 a_{2}^{2}\right) u^{4}+\frac{1}{216} a_{3}^{3} u^{3} .
\end{aligned}
$$

It is an amusing exercise to see that under the $\operatorname{limit} \operatorname{Im} \tau \rightarrow+\infty$ :

$$
\wp(\tau \mid \zeta) \rightarrow-\frac{\pi^{2}}{3}+\frac{\pi^{2}}{\sin ^{2}(\pi \zeta)}, \quad \wp^{\prime}(\tau \mid \zeta) \rightarrow-\frac{2 \pi^{3} \cos (\pi \zeta)}{\sin ^{3}(\pi \zeta)}
$$

the curve above reduces to the trigonometric one with three Wilson lines $[28,(2.8)]$.

Three Wilson lines II If we consider the model with $\mu=m_{1}\left(\mathbf{e}_{1}-\mathbf{e}_{2}\right)+m_{2}\left(\mathbf{e}_{3}-\mathbf{e}_{4}\right)+$ $m_{3}\left(\mathbf{e}_{5}-\mathbf{e}_{6}\right)$, the nine base points of the cubic pencil are given by $\left\{0,0,0, \pm m_{1}, \pm m_{2}, \pm m_{3}\right\}$. As the line intersecting with $E_{\infty}$ with the three points $\left\{0, \pm m_{i}\right\}$ is given by $x_{1}-\wp_{i} x_{0}=0$, where we set $\wp_{i}:=\wp\left(\tau \mid m_{i}\right)$, the cubic $Q$ we need is found to be

$$
Q=\left(x_{1}-\wp_{1} x_{0}\right)\left(x_{1}-\wp_{2} x_{0}\right)\left(x_{1}-\wp_{3} x_{0}\right)
$$

The computation of the algebraic invariants of the cubic pencil $P+t Q$ yields the Weierstrass form (8.1) with

$$
\begin{aligned}
f\left(u ; \tau, m_{i}\right) & =f_{0} u^{4}+\frac{1}{4}\left(4 \sigma_{2}-f_{0}\right) u^{3}+\frac{1}{12}\left(\sigma_{1}^{2}-3 \sigma_{2}\right) u^{2} \\
g\left(u ; \tau, m_{i}\right) & =g_{0} u^{6}-\frac{1}{12}\left(f_{0} \sigma_{1}+\sigma_{3}\right) u^{5}+\frac{1}{48}\left(3 g_{0}+f_{0} \sigma_{1}-4 \sigma_{1} \sigma_{2}\right) u^{4} \\
& -\frac{1}{432}\left(2 \sigma_{1}^{3}-9 \sigma_{1} \sigma_{2}+27 \sigma_{3}\right) u^{3}
\end{aligned}
$$

where $\sigma_{1}=\wp_{1}+\wp_{2}+\wp_{3}, \sigma_{2}=\wp_{1} \wp_{2}+\wp_{2} \wp_{3}+\wp_{3} \wp_{1}$, and $\sigma_{3}=\wp_{1} \wp_{2} \wp_{3}$. 
Four Wilson lines Let us consider the model with the Wilson lines given in the Euclidean coordinates $\mu=\sum_{i=1}^{4} m_{i} \mathbf{e}_{i}$, where any of $m_{i}$ or $m_{i}-m_{j}$ is non-zero. After a suitable $W\left(\boldsymbol{E}_{8}\right)$ action, the nine base points of the cubic pencil become $\left\{\nu_{i}\right\}=$ $\left\{-2 \zeta_{0}, \zeta_{0}, \zeta_{0}, \zeta_{0}, \zeta_{0}, \zeta_{1}, \zeta_{2}, \zeta_{3}, \zeta_{4}\right\}$, where

$$
\left(\zeta_{0}, \zeta_{1}, \zeta_{2}, \zeta_{3}, \zeta_{4}\right)=\frac{1}{6}\left(m_{1}, m_{2}, m_{3}, m_{4}\right)\left(\begin{array}{rrrrr}
1 & -5 & 1 & 1 & 1 \\
1 & 1 & -5 & 1 & 1 \\
1 & 1 & 1 & -5 & 1 \\
1 & 1 & 1 & 1 & -5
\end{array}\right) .
$$

The first three points $\left\{-2 \zeta_{0}, \zeta_{0}, \zeta_{0}\right\}$ defines a line $L$ tangent to the elliptic curve $E_{\infty}$, while the remaining six a conic $C$. If we set $\wp_{i}=\wp\left(\tau \mid \zeta_{i}\right), \wp_{i}^{\prime}=\wp^{\prime}\left(\tau \mid \zeta_{i}\right), \wp_{00}=\wp\left(\tau \mid 2 \zeta_{0}\right)$, $\wp_{00}^{\prime}=-\wp^{\prime}\left(\tau \mid 2 \zeta_{0}\right)$, they are given by

$$
\begin{aligned}
L\left(x_{0}, x_{1}, x_{2}\right) & =\left|\begin{array}{ccc}
x_{0} & x_{1} & x_{2} \\
1 & \wp_{0} & \wp_{0}^{\prime} \\
1 & \wp_{00} & \wp_{00}^{\prime}
\end{array}\right|, \\
C\left(x_{0}, x_{1}, x_{2}\right) & =\left|\begin{array}{cccccc}
x_{0}^{2} & x_{0} x_{1} & x_{0} x_{2} & x_{1}^{2} & x_{1} x_{2} & x_{2}^{2} \\
1 & \wp_{0} & \wp_{0}^{\prime} & \wp_{0}^{2} & \wp_{0} \wp_{0}^{\prime} & \left(\wp_{0}^{\prime}\right)^{2} \\
1 & \wp_{1} & \wp_{1}^{\prime} & \wp_{1}^{2} & \wp_{1} \wp_{1}^{\prime} & \left(\wp_{1}^{\prime}\right)^{2} \\
1 & \wp_{2} & \wp_{2}^{\prime} & \wp_{2}^{2} & \wp_{2} \wp_{2}^{\prime} & \left(\wp_{2}^{\prime}\right)^{2} \\
1 & \wp_{3} & \wp_{3}^{\prime} & \wp_{3}^{2} & \wp_{3} \wp_{3}^{\prime} & \left(\wp_{3}^{\prime}\right)^{2} \\
1 & \wp_{4} & \wp_{4}^{\prime} & \wp_{4}^{2} & \wp_{4} \wp_{4}^{\prime} & \left(\wp_{4}^{\prime}\right)^{2}
\end{array}\right| .
\end{aligned}
$$

The cubic in question is found to be $Q=L \cdot C$. However, the Weierstrass form of the cubic pencil $P+t Q$ is so complicated that we do not attempt to describe it here.

Four Wilson lines II Finally, let us take the model with $\left\{\nu_{i}\right\}=\left\{0, \pm \zeta_{1}, \pm \zeta_{2}, \pm \zeta_{3}, \pm \zeta_{4}\right\}$. We thus need to find a cubic $Q$ which passes through the eight points $\left(1, \pm \wp_{i}, \wp_{i}^{\prime}\right)$, where $\wp_{i}=\wp\left(\tau \mid \zeta_{i}\right), \wp_{i}^{\prime}=\wp^{\prime}\left(\tau \mid \zeta_{i}\right)$, for $i=1, \ldots, 4$, as well as $(0,0,1)$.

We find that such a cubic $Q$ is given by

$$
Q=\left(g_{0}-f_{0} \sigma_{1}-4 \sigma_{3}\right) x_{0}^{2} x_{1}+\left(f_{0}+4 \sigma_{2}\right) x_{0} x_{1}^{2}-\sigma_{1} x_{0} x_{2}^{2}+x_{1} x_{2}^{2}+\left(4 \sigma_{4}-g_{0} \sigma_{1}\right) x_{0}^{3},
$$

where $\sigma_{i}$ is the $i$ th basic symmetric polynomial of $\wp_{i}$ s. In fact we can see that

$$
Q(1, x, y)=4\left(x-\wp_{1}\right)\left(x-\wp_{2}\right)\left(x-\wp_{3}\right)\left(x-\wp_{4}\right),
$$

if $y^{2}=4 x^{3}-f_{0} x-g_{0}$, that is, if we restrict $Q$ on $E_{\infty}$. Nevertheless, $Q$ itself is an irreducible cubic in this case. 


\section{Acknowledgements.}

I benefitted from attending the workshop "Periods Associated to Rational Elliptic Surfaces and Elliptic Lie Algebras" held at International Christian University in June 2001. I wish to express my gratitude to many participants both of the workshop and of my informal talks at Nagoya University for useful comments. Among them are Profs. H. Kanno, A. Kato, S. Kondo, H. Ohta, Y. Shimizu, A. Tsuchiya, and Y. Yamada. I would also like to thank Dr. Y. Ohtake for helpful discussions. My special thanks are due to the late Prof. S.-K. Yang for valuable advice. This work was supported in part by Grant-in-Aid for Scientific Research on Priority Area 707 "Supersymmetry and Unified Theory of Elementary Particles", Japan Ministry of Education, Science, Sports, Culture and Technology.

\section{Appendix A}

In this appendix we present for completeness the explicit form of the generators $\mathcal{S}$ and $\mathcal{T}$ of the algebraic invariants of the ternary cubic form $R(9.3)$.

$$
\begin{aligned}
\mathcal{S} & =-a_{300} a_{120} a_{012}^{2}-2 a_{210} a_{012} a_{111}^{2}+a_{300} a_{030} a_{102} a_{012}+a_{300} a_{003} a_{120} a_{021} \\
& +a_{030} a_{003} a_{210} a_{201}-a_{210} a_{120} a_{102} a_{012}-a_{210} a_{201} a_{012} a_{021}-a_{120} a_{201} a_{102} a_{021} \\
& -a_{030} a_{201}^{2} a_{012}-a_{003} a_{120}^{2} a_{201}-a_{300} a_{102} a_{021}^{2}+a_{210}^{2} a_{012}^{2}-a_{030} a_{210} a_{102}^{2} \\
& -a_{003} a_{210}^{2} a_{021}+a_{120}^{2} a_{102}^{2}+a_{201}^{2} a_{021}^{2}-2 a_{120} a_{102} a_{111}^{2}+a_{111}^{4} \\
& -a_{300} a_{030} a_{003} a_{111}-2 a_{201} a_{021} a_{111}^{2}+a_{300} a_{012} a_{021} a_{111}+a_{030} a_{201} a_{102} a_{111} \\
& +a_{003} a_{210} a_{120} a_{111}+3 a_{210} a_{102} a_{021} a_{111}+3 a_{120} a_{201} a_{012} a_{111}, \\
\mathcal{T} & =-3 a_{012}^{2} a_{300}^{2} a_{021}^{2}-24 a_{111}^{2} a_{201}^{2} a_{021}^{2}+24 a_{120} a_{111}^{4} a_{102}+4 a_{120}^{3} a_{300} a_{003}^{2} \\
& -3 a_{120}^{2} a_{210}^{2} a_{003}^{2}-27 a_{210}^{2} a_{102}^{2} a_{021}^{2}+4 a_{201}^{3} a_{030}^{2} a_{003}-24 a_{012}^{2} a_{210}^{2} a_{111}^{2} \\
& +24 a_{012} a_{210} a_{111}^{4}-24 a_{120}^{2} a_{102}^{2} a_{111}^{2}+4 a_{300}^{2} a_{021}^{3} a_{003}+a_{300}^{2} a_{030}^{2} a_{003}^{2} \\
& +24 a_{111}^{4} a_{201} a_{021}-12 a_{120}^{2} a_{300} a_{003} a_{102} a_{021}+4 a_{012}^{3} a_{300}^{2} a_{030}-27 a_{012}^{2} a_{120}^{2} a_{201}^{2} \\
& -3 a_{201}^{2} a_{030}^{2} a_{102}^{2}+8 a_{120}^{3} a_{102}^{3}+8 a_{012}^{3} a_{210}^{3}+24 a_{210}^{2} a_{003} a_{201} a_{021}^{2} \\
& +4 a_{300} a_{030}^{2} a_{102}^{3}+12 a_{210}^{2} a_{111}^{2} a_{021} a_{003}+12 a_{210} a_{111}^{2} a_{102}^{2} a_{030}-36 a_{210} a_{111}^{3} a_{021} a_{102}
\end{aligned}
$$




$$
\begin{aligned}
& +8 a_{201}^{3} a_{021}^{3}-8 a_{111}^{6}+4 a_{210}^{3} a_{003}^{2} a_{030}-24 a_{300} a_{030} a_{102}^{2} a_{111} a_{021} \\
& +12 a_{201}^{2} a_{030} a_{102} a_{111} a_{021}-12 a_{120} a_{210} a_{102}^{3} a_{030}-12 a_{120} a_{210} a_{111}^{3} a_{003} \\
& +24 a_{120} a_{300} a_{102}^{2} a_{021}^{2}-12 a_{300} a_{201} a_{021}^{3} a_{102}-12 a_{102} a_{201} a_{111}^{3} a_{030} \\
& -24 a_{210}^{2} a_{003} a_{102} a_{111} a_{030}-24 a_{300} a_{210} a_{021}^{2} a_{111} a_{003}-20 a_{300} a_{111}^{3} a_{030} a_{003} \\
& -12 a_{201}^{2} a_{030} a_{210} a_{021} a_{003}+36 a_{210} a_{111}^{2} a_{201} a_{030} a_{003}+36 a_{210} a_{102} a_{111} a_{021}^{2} a_{201} \\
& +6 a_{300} a_{030} a_{102} a_{210} a_{021} a_{003}-6 a_{300} a_{030}^{2} a_{102} a_{201} a_{003}+6 a_{210} a_{201} a_{021} a_{102}^{2} a_{030} \\
& +12 a_{300} a_{021}^{2} a_{111}^{2} a_{102}+12 a_{300} a_{201} a_{030} a_{003} a_{111} a_{021}+12 a_{012} a_{300} a_{210} a_{030} a_{111} a_{003} \\
& +6 a_{012} a_{300} a_{210} a_{021}^{2} a_{102}+12 a_{012} a_{120} a_{210}^{2} a_{111} a_{003}+6 a_{012} a_{120} a_{201}^{2} a_{030} a_{102} \\
& +18 a_{012} a_{300} a_{030} a_{102} a_{201} a_{021}+6 a_{012} a_{120} a_{300} a_{201} a_{030} a_{003}-12 a_{012} a_{120} a_{300} a_{030} a_{102}^{2} \\
& -12 a_{012} a_{120} a_{210} a_{111}^{2} a_{102}+36 a_{012} a_{120} a_{201}^{2} a_{021} a_{111}+36 a_{012} a_{120}^{2} a_{111} a_{102} a_{201} \\
& -24 a_{012} a_{120}^{2} a_{300} a_{111} a_{003}+6 a_{012} a_{120}^{2} a_{210} a_{201} a_{003}+18 a_{012} a_{120} a_{300} a_{210} a_{021} a_{003} \\
& -60 a_{012} a_{120} a_{300} a_{111} a_{021} a_{102}+6 a_{012}^{2} a_{120} a_{300} a_{201} a_{021}-24 a_{012}^{2} a_{300} a_{201} a_{111} a_{030} \\
& -6 a_{012} a_{120} a_{210} a_{102} a_{201} a_{021}-12 a_{012} a_{210} a_{111}^{2} a_{201} a_{021}+36 a_{012} a_{300} a_{030} a_{102} a_{111}^{2} \\
& +36 a_{012}^{2} a_{120} a_{210} a_{111} a_{201}-12 a_{012}^{2} a_{300} a_{030} a_{102} a_{210}+12 a_{012}^{2} a_{300} a_{111} a_{021} a_{210} \\
& -24 a_{120} a_{201}^{2} a_{030} a_{111} a_{003}-12 a_{120} a_{102} a_{201}^{2} a_{021}^{2}+36 a_{120} a_{210} a_{102}^{2} a_{111} a_{021} \\
& +6 a_{120} a_{210}^{2} a_{003} a_{102} a_{021}-60 a_{012} a_{102} a_{201} a_{111} a_{030} a_{210}+36 a_{012} a_{210}^{2} a_{111} a_{021} a_{102} \\
& +12 a_{120} a_{300} a_{030} a_{102} a_{111} a_{003}-60 a_{120} a_{210} a_{201} a_{021} a_{111} a_{003}+18 a_{120} a_{210} a_{102} a_{201} a_{030} a_{003} \\
& -12 a_{120} a_{102} a_{111}^{2} a_{201} a_{021}+12 a_{120} a_{201} a_{111} a_{030} a_{102}^{2}-12 a_{120}^{3} a_{102} a_{201} a_{003} \\
& -12 a_{120} a_{300} a_{003} a_{201} a_{021}^{2}+36 a_{120} a_{300} a_{111}^{2} a_{021} a_{003}-6 a_{120} a_{300} a_{003}^{2} a_{210} a_{030} \\
& -12 a_{120}^{2} a_{102}^{2} a_{201} a_{021}+24 a_{120}^{2} a_{201}^{2} a_{021} a_{003}+12 a_{120}^{2} a_{111}^{2} a_{201} a_{003} \\
& +12 a_{012} a_{201}^{2} a_{030} a_{111}^{2}-12 a_{012} a_{300} a_{111}^{3} a_{021}-12 a_{012} a_{120}^{2} a_{210} a_{102}^{2}-12 a_{012} a_{201}^{3} a_{030} a_{021} \\
& -12 a_{012} a_{210} a_{201}^{2} a_{021}^{2}-36 a_{012} a_{120} a_{111}^{3} a_{201}-12 a_{012} a_{210}^{3} a_{021} a_{003}+24 a_{012} a_{210}^{2} a_{102}^{2} a_{030} \\
& -12 a_{012}^{3} a_{300} a_{210} a_{120}+24 a_{012}^{2} a_{120}^{2} a_{300} a_{102}-12 a_{012}^{2} a_{120} a_{210}^{2} a_{102}+12 a_{012}^{2} a_{120} a_{300} a_{111}^{2} \\
& -12 a_{012}^{2} a_{210}^{2} a_{201} a_{021}+24 a_{012}^{2} a_{201}^{2} a_{030} a_{210}+12 a_{120}^{2} a_{210} a_{102} a_{111} a_{003} \\
& -12 a_{012} a_{210}^{2} a_{201} a_{030} a_{003}+12 a_{012} a_{300} a_{201} a_{021}^{2} a_{111}-6 a_{012} a_{300}^{2} a_{030} a_{021} a_{003} \text {. }
\end{aligned}
$$




\section{References}

[1] G. Aldazabal, A. Font, L. E. Ibáñez and A. M. Uranga, New Branches of String Compactifications and their F-Theory Duals, Nucl. Phys. B492 (1996) 119-151, hep-th/9607121.

[2] M. Bershadsky, S. Cecotti, H. Ooguri and C. Vafa, Holomorphic Anomalies in Topological Field Theories, Nucl. Phys. B405 (1993) 279-304, hep-th/9302103.

[3] M. Bershadsky, S. Cecotti, H. Ooguri and C. Vafa, Kodaira-Spencer Theory of Gravity and Exact Results for Quantum String Amplitudes, Commun. Math. Phys. 165 (1994) 311-427, hep-th/9309140.

[4] J. Bryan and R. Pandharipande, BPS States of Curves in Calabi-Yau 3-Folds, Geometry and Topology 5 (2001) 287-318, math.AG/0009025.

[5] P. Candelas, X. C. de la Ossa, P. S. Green and L. Parkes, A Pair of Calabi-Yau Manifolds as an Exactly Soluble Superconformal Theory, Nucl. Phys. B359 (1991) $21-74$.

[6] T.-M. Chiang, A. Klemm, S.-T. Yau and E. Zaslow, Local Mirror Symmetry: Calculations and Interpretations, Adv. Theor. Math. Phys. 3 (1999) 495-565, hepth/9903053.

[7] G. Curio and D. Lüst, A Class of $N=1$ Dual String Pairs and Its Modular Superpotentials, Int. J. Mod. Phys. A12 (1997) 5847-5866, hep-th/9703007.

[8] O. DeWolfe, T. Hauer, A. Iqbal and B. Zwiebach, Uncovering Infinite Symmetries on $[p, q]$ 7-Branes: Kac-Moody Algebras and Beyond, Adv. Theor. Math. Phys. 3 (1999) 1835-1891, hep-th/9812209.

[9] M. Fukae, Y. Yamada and S.-K. Yang, Mordell-Weil Lattice via String Junctions, Nucl. Phys. B572 (2000) 71-94, hep-th/9909122.

[10] O. J. Ganor, Toroidal Compactification of Heterotic 6D Non-Critical Strings down to Four Dimensions, Nucl. Phys. B488 (1997) 223-235, hep-th/9608109. 
[11] O. J. Ganor and A. Hanany, Small $E_{8}$ Instantons and Tensionless Non-Critical Strings, Nucl. Phys. B474 (1996) 122-138, hep-th/9602120.

[12] O. J. Ganor, D. R. Morrison and N. Seiberg, Branes, Calabi-Yau Spaces, and Toroidal Compactification of the $N=1$ Six-Dimensional $E_{8}$ Theory, Nucl. Phys. B487 (1997) 93-127, hep-th/9610251.

[13] R. Gopakumar and C. Vafa, M-Theory and Topological Strings-II, hep-th/9812127.

[14] T. Hauer and A. Iqbal, Del Pezzo Surfaces and Affine 7-Brane Backgrounds, J. High Energy Phys. 0001 (2000) 043, hep-th/9910054.

[15] D. Hilbert, Theory of Algebraic Invariants, Cambridge Mathematical Library, Cambridge Univ. Press, 1993.

[16] S. Hosono, A. Klemm, S. Theisen and S.-T. Yau, Mirror Symmetry, Mirror Map and Applications to Complete Intersection Calabi-Yau Spaces, Nucl. Phys. B433 (1995) 501-552, hep-th/9406055.

[17] S. Hosono, M.-H. Saito and J. Stienstra, On the Mirror Symmetry Conjecture for Schoen's Calabi-Yau 3-Folds, in Integrable System and Algebraic Geometry, Proc. Taniguchi Symposium 1997, M.-H. Saito, Y. Shimizu and K. Ueno (eds.), World Scientific 1998, 194-235, alg-geom/9709027.

[18] S. Hosono, M.-H. Saito and A. Takahashi, Holomorphic Anomaly Equation and BPS State Counting of Rational Elliptic Surface, Adv. Theor. Math. Phys. 3 (1999) 177208, hep-th/9901151.

[19] S. Hosono, M.-H. Saito and A. Takahashi, Relative Lefschetz Action and BPS State Counting, Int. Math. Res. Notice. 15 (2001) 783-816, math.AG/0105148.

[20] A. Hurwitz und R. Courant, Vorlesungen über Allgemeine Funktionentheorie und Elliptische Funktionen, Die Grundlehren der mathematischen Wissenschaften, Bd. 3, Springer-Verlag, 1929. 
[21] S. Katz, A. Klemm and C. Vafa, M-Theory, Topological Strings and Spinning Black Holes, Adv. Theor. Math. Phys. 3 (1999) 1445-1537, hep-th/9910181.

[22] T. Kawai and K. Yoshioka, String Partition Functions and Infinite Products, Adv. Theor. Math. Phys. 4 (2000) 397-485, hep-th/0002169.

[23] A. Klemm, P. Mayr and C. Vafa, BPS States of Exceptional Non-Critical Strings, Nucl. Phys. B (Proc. Suppl.) 58 (1997) 177-194, hep-th/9607139.

[24] W. Lerche, P. Mayr and N. P. Warner, Non-Critical Strings, del Pezzo Singularities and Seiberg-Witten Curves, Nucl. Phys. B499 (1997) 125-148, hep-th/9612085.

[25] B. H. Lian and S.-T. Yau, Arithmetic Properties of Mirror Map and Quantum Coupling, Commun. Math. Phys. 176 (1996) 163-191, hep-th/9411234.

[26] E. Looijenga, Rational Surfaces with an Anti-Canonical Cycle, Ann. Math. 114 (1981) 267-322.

[27] J. A. Minahan, D. Nemeschansky, C. Vafa and N. P. Warner, E-Strings and $N=4$ Topological Yang-Mills Theories, Nucl. Phys. B527 (1998) 581-623, hep-th/9802168.

[28] J. A. Minahan, D. Nemeschansky and N. P. Warner, Investigating the BPS Spectrum of Non-Critical $E_{n}$ Strings, Nucl. Phys. B508 (1997) 64-106, hep-th/9705237.

[29] J. A. Minahan, D. Nemeschansky and N. P. Warner, Partition Functions for BPS States of the Non-Critical E $E_{8}$ String, Adv. Theor. Math. Phys. 1 (1998) 167-183, hep-th/9707149.

[30] R. Miranda and U. Persson, On Extremal Rational Elliptic Surfaces, Math. Z. 193 (1986) 537-558.

[31] K. Mohri, Y. Ohtake and S.-K. Yang, Dualities between String Junctions and DBranes on del Pezzo Surfaces, Nucl. Phys. B595 (2001) 138-164, hep-th/0007243.

[32] K. Mohri, Y. Onjo and S.-K. Yang, Closed Sub-Monodromy Problems, Local Mirror Symmetry and Branes on Orbifolds, Rev. Math. Phys. 13 No. 6 (2001) 675-715, hep-th/0009072. 
[33] K. Saito, Extended Affine Root Systems II (Flat Invariants), Publ. RIMS, Kyoto Univ. 26 (1990) 15-78.

[34] H. Sakai, Rational Surfaces with Affine Root Systems and Geometry of the Painlevé Equations, Commun. Math. Phys. 220 (2001) 165-221.

[35] I. Satake, Automorphisms of the Extended Affine Root Systems and Modular Property for the Flat Theta Invariants, Publ. RIMS, Kyoto Univ. 31 (1995) 1-32.

[36] A. N. Schellekens and N. P. Warner, Anomalies, Characters and Strings, Nucl. Phys. B287 (1987) 317-361.

[37] B. Schoeneberg, Elliptic Modular Functions: An Introduction, Die Grundlehren der mathematischen Wissenschaften, Bd. 203, Springer-Verlag, 1974.

[38] N. Seiberg and E. Witten, Monopoles, Duality and Chiral Symmetry Breaking in $N=2$ Supersymmetric QCD, Nucl. Phys. B431 (1994) 484-550, hep-th/9408099.

[39] B. Sturmfels, Algorithms in Invariant Theory, Texts and Monographs in Symbolic Computation, Springer, 1993.

[40] J. Tannery et J. Molk, Éléments de la Théorie des Fonctions Elliptiques, Tomes I-IV, Gauthier-Villars, 1893-1902; reprinted in two vols. Chelsea, New York, 1972.

[41] K. Wirthmüller, Root Systems and Jacobi Forms, Compositio Math. 82 (1992) 293354. 